\title{
A Survey on Some Anisotropic Hardy-Type Function Spaces
}

\author{
Jun $\mathrm{Liu}^{1}$, Dorothee D. Haroske ${ }^{2}$ and Dachun Yang ${ }^{3, *}$ \\ 1 School of Mathematics, China University of Mining and Technology, Xuzhou, \\ Jiangsu 221116, China \\ 2 Institute of Mathematics, Friedrich Schiller University Jena, Jena 07737, Germany \\ ${ }^{3}$ Laboratory of Mathematics and Complex Systems (Ministry of Education of China), \\ School of Mathematical Sciences, Beijing Normal University, Beijing 100875, China
}

Received 10 December 2019; Accepted (in revised version) 3 January 2020

Dedicated to Professor Weiyi Su on the occasion of her 80th birthday

\begin{abstract}
Let $A$ be a general expansive matrix on $\mathbb{R}^{n}$. The aims of this article are twofold. The first one is to give a survey on the recent developments of anisotropic Hardy-type function spaces on $\mathbb{R}^{n}$, including anisotropic Hardy-Lorentz spaces, anisotropic variable Hardy spaces and anisotropic variable Hardy-Lorentz spaces as well as anisotropic Musielak-Orlicz Hardy spaces. The second one is to correct some errors and seal some gaps existing in the known articles. Some unsolved problems are also presented.
\end{abstract}

Key Words: Expansive matrix, (variable) Hardy space, (variable) Hardy-Lorentz space, MusielakOrlicz Hardy space.

AMS Subject Classifications: 42B35, 42B30, 42B25, 46B70

\section{Introduction}

In order to meet the requirements arising in the development of harmonic analysis and partial differential equations, there has been more and more research in extending classical function spaces from Euclidean spaces to some more general underlying spaces; see, for instance, $[8,34,44,47,49,58,85,90,116]$. In 2003, to give a unified framework of the real-variable theory of both the isotropic Hardy space and the parabolic Hardy space of Calderón and Torchinsky [19], for the first time, Bownik [12] introduced the anisotropic Hardy space $H_{A}^{p}\left(\mathbb{R}^{n}\right)$ with $p \in(0, \infty)$, where $A$ is a general expansive matrix on $\mathbb{R}^{n}$ (see [12, p. 5, Definition 2.1]). In [12], Bownik also established the characterizations

*Corresponding author. Email addresses: junliu@cumt.edu.cn (J. Liu), dorothee.haroske@uni-jena.de (D. D. Haroske), dcyang@bnu.edu.cn (D. Yang) 
of $H_{A}^{p}\left(\mathbb{R}^{n}\right)$, respectively, in terms of atoms, maximal functions and tight frame multiwavelets (see [12, p. 94, Definition 4.2]), and proved as well that the dual space of $H_{A}^{p}\left(\mathbb{R}^{n}\right)$ with $p \in(0,1]$ is the anisotropic Campanato space; as applications, Bownik [12] also obtained the boundedness of anisotropic Calderón-Zygmund operators from $H_{A}^{p}\left(\mathbb{R}^{n}\right)$ to itself (or to the Lebesgue space $L^{p}\left(\mathbb{R}^{n}\right)$ ). Later on, Bownik et al. [16] further extended the anisotropic Hardy space to the weighted setting. Very recently, Wang [110] considered a multiplier theorem on anisotropic Hardy spaces $H_{A}^{p}\left(\mathbb{R}^{n}\right)$. Nowadays, the anisotropic setting has proved useful not only in developing function spaces, but also in many other branches such as the wavelet theory (see, for instance, $[5,12,25]$ ) and partial differential equations (see, for instance, $[18,53]$ ).

Let us briefly recall some history of the study of anisotropic function spaces. It has been developed parallel to the theory for isotropic spaces; we refer the reader in particular to the monographs $[9,88]$ (and the articles mentioned there), and to the survey [10]. For any $p \in(1, \infty)$ and $\left\{s_{i}\right\}_{i=1}^{n} \subset \mathbb{N}$, the (classical) anisotropic Sobolev space on $\mathbb{R}^{n}$ contains all $f \in L^{p}\left(\mathbb{R}^{n}\right)$ such that

$$
\frac{\partial^{s_{i}} f}{\partial x_{i}^{s_{i}}} \in L^{p}\left(\mathbb{R}^{n}\right) \quad \text { for any } i \in\{1, \cdots, n\} .
$$

It is obvious that, unlike in case of the isotropic Sobolev space (namely, the case when $\left.s_{1}=\cdots=s_{n}\right)$, the smoothness properties of an element depend on the chosen direction in $\mathbb{R}^{n}$. The number $s$, defined by setting $\frac{1}{s}:=\frac{1}{n}\left(\frac{1}{s_{1}}+\cdots+\frac{1}{s_{n}}\right)$, is usually called the mean smoothness, and $a=\left(a_{1}, \cdots, a_{n}\right)$, given by $a_{i}:=\frac{s}{s_{i}}, i \in\{1, \cdots, n\}$, characterizes the anisotropy. Similarly to the isotropic situation, more general scales of anisotropic Bessel potential spaces (fractional Sobolev spaces), anisotropic Besov spaces and anisotropic Triebel-Lizorkin spaces were studied. It is well known that the isotropic theory has a more or less complete counterpart of the fundamentals (definitions, description via differences and derivatives, elementary properties, embeddings for different metrics, interpolation) in the context of anisotropic spaces. A survey on the basic results for the (anisotropic) spaces of Besov or Triebel-Lizorkin type was given in [94, Subsections 4.2.1 through 4.2.4] (with preceding results in [83,92,93, 103-106]) and [57, Sections 2.1 and 2.2]. More recently, several authors were concerned with the problem of obtaining useful decompositions of anisotropic function spaces of Besov and Triebel-Lizorkin type. A construction of unconditional bases using Meyer wavelets was obtained in $[7,8]$; see also $[44,45,49]$; a different approach, involving the $\varphi$-transform of Frazier and Jawerth (see [40,41]) was followed in [28-30]; see also [95]. More recent contributions can be found in [13-15] and [55, 61,62]. Based on the approach used in $[107,108]$, further representations were obtained by local means, atomic and sub-atomic decompositions, which can be found in [34,47]; see also [24,35,36,101,112,113] for applications. Finally, let us refer the reader to [109, Chapter 5] where Triebel gave a very nice and detailed summary of the history, recent developments and the state-of-the-art (in 2006), which we also recommend for further references. Moreover, Barrios et al. [4] further characterized the anisotropic Besov spaces in terms of Peetre maximal functions and approximations; Li et 
al. [64] presented the duality theory of weighted anisotropic Besov and Triebel-Lizorkin spaces; Li et al. [65] and Liu et al. [80,81] established the characterizations of weighted anisotropic Besov and Triebel-Lizorkin spaces via Littlewood-Paley functions involving ball averages. In addition, anisotropic weak Hardy spaces were studied by Ding et al. [27] and Barrios et al. [5], which were just special cases of anisotropic Hardy-Lorentz spaces introduced by Liu et al. in [77, 79]; anisotropic local Hardy spaces were investigated by Betancor et al. [11]; weighted anisotropic product Hardy spaces were considered by Bownik et al. [17] and Li et al. [63]. In 2014, based on the work of both Bownik [12] and Ky [60], Li et al. [68] introduced the anisotropic Musielak-Orlicz Hardy space, which was a generalization of the anisotropic Hardy space of Bownik [12], the weighted anisotropic Hardy space of Bownik et al. [16] as well as the Musielak-Orlicz Hardy space of Ky [60]. Recently, the anisotropic product Musielak-Orlicz Hardy space was studied by Fan et al. in [32] and the anisotropic mixed-norm Hardy space by Huang et al. in [51].

The main purposes of this article are twofold. The first one is to give a survey on the recent developments of anisotropic Hardy-type function spaces on $\mathbb{R}^{n}$, including anisotropic Hardy-Lorentz spaces, anisotropic variable Hardy spaces, anisotropic variable Hardy-Lorentz spaces and anisotropic Musielak-Orlicz Hardy spaces. To be precise, the main results that we review include: various real-variable characterizations of these four kinds of function spaces, the boundedness of Calderón-Zygmund operators on anisotropic Hardy-Lorentz spaces and Musielak-Orlicz Hardy spaces, the boundedness of maximal operators of the Bochner-Riesz and the Weierstrass means on anisotropic variable Hardy spaces and Hardy-Lorentz spaces as well as some real interpolation results. The second purpose is to correct some errors and seal some gaps existing in the proofs of Lusin area function characterizations of the above four kinds of function spaces, namely, the proofs of the sufficiencies of, respectively, [79, Theorem 2.7], [75, Theorem 6.1], [78, Theorem 5.2] and [67, Theorem 2.8]. In addition, some unsolved problems are also presented.

The organization of this survey is as follows.

In Section 2, we first give some notation which are used throughout this article and then recall some notions on expansive matrices and homogeneous quasi-norms.

The aim of Section 3 is the summary of anisotropic Hardy-Lorentz spaces $H_{A}^{p, q}\left(\mathbb{R}^{n}\right)$ with $p \in(0, \infty), q \in(0, \infty]$ and $A$ being a general expansive matrix. To this end, we first recall the notion of Lorentz spaces $L^{p, q}\left(\mathbb{R}^{n}\right)$ which are then used to define $H_{A}^{p, q}\left(\mathbb{R}^{n}\right)$. Moreover, in Subsections 3.2 and 3.3, various real-variable characterizations of the spaces $H_{A}^{p, q}\left(\mathbb{R}^{n}\right)$, respectively, in terms of maximal functions, atoms, finite atoms, molecules and the Lusin area functions as well as the Littlewood-Paley $g$-functions or $g_{\lambda}^{*}$-functions, established in $[77,79]$, are presented. Some errors and gaps existing in the proof of the sufficiency of [79, Theorem 2.7] are also corrected and sealed in Subsection 3.3. As an application, the fact that the space $H_{A}^{p, q}\left(\mathbb{R}^{n}\right)$ is an intermediate space between $H_{A}^{p_{1}, q_{1}}\left(\mathbb{R}^{n}\right)$ and $H_{A}^{p_{2}, q_{2}}\left(\mathbb{R}^{n}\right)$ with $0<p_{1}<p<p_{2}<\infty$ and $q_{1}, q, q_{2} \in(0, \infty]$, and also between $H_{A}^{p, q_{1}}\left(\mathbb{R}^{n}\right)$ and $H_{A}^{p, q_{2}}\left(\mathbb{R}^{n}\right)$ with $p \in(0, \infty)$ and $0<q_{1}<q<q_{2} \leq \infty$ in the real method of interpolation, proved in [77, Theorem 6.1], is displayed. As another application, the bounded- 
ness of $\delta$-type Calderón-Zygmund operators from $H_{A}^{p}\left(\mathbb{R}^{n}\right)$ to the weak Lebesgue space $L^{p, \infty}\left(\mathbb{R}^{n}\right)$ (or to $H_{A}^{p, \infty}\left(\mathbb{R}^{n}\right)$ ) in the critical case, from $H_{A}^{p, q}\left(\mathbb{R}^{n}\right)$ to itself (or to $L^{p, q}\left(\mathbb{R}^{n}\right)$ ) with $\delta \in\left(0, \frac{\ln \lambda_{-}}{\ln b}\right], p \in\left(\frac{1}{1+\delta}, 1\right]$ and $q \in(0, \infty]$, as well as the boundedness of some CalderónZygmund operators from $H_{A}^{p, q}\left(\mathbb{R}^{n}\right)$ to $L^{p, \infty}\left(\mathbb{R}^{n}\right)$, obtained in [77, Subsection 6.2], are also given, where $b:=|\operatorname{det} A|, \lambda_{-} \in(1, \min \{|\lambda|: \lambda \in \sigma(A)\}]$ and $\sigma(A)$ denotes the set of all eigenvalues of $A$.

In Section 4, we give the real-variable theory of anisotropic variable Hardy spaces $H_{A}^{p(\cdot)}\left(\mathbb{R}^{n}\right)$, where the variable exponent function, $p(\cdot): \mathbb{R}^{n} \rightarrow(0, \infty]$, satisfies the socalled globally log-Hölder continuity condition (see $[75,(2.5)$ and (2.6)]). For this purpose, we first recall the definition of variable Lebesgue spaces $L^{p(\cdot)}\left(\mathbb{R}^{n}\right)$, which are the associated basic function spaces of $H_{A}^{p(\cdot)}\left(\mathbb{R}^{n}\right)$, and then present the notion of the spaces $H_{A}^{p(\cdot)}\left(\mathbb{R}^{n}\right)$. Furthermore, we display various real-variable characterizations of the spaces $H_{A}^{p(\cdot)}\left(\mathbb{R}^{n}\right)$ from [75], respectively, by means of maximal functions, atoms, finite atoms, and the Lusin area functions as well as the Littlewood-Paley $g$-functions or $g_{\lambda}^{*}$-functions. Some errors and gaps existing in the proof of the sufficiency of [75, Theorem 6.1] are also corrected and sealed in Subsection 4.3. As applications, a criterion on the boundedness of some sublinear operators from $H_{A}^{p(\cdot)}\left(\mathbb{R}^{n}\right)$ into a quasi-Banach space and the boundedness of maximal operators of the $\theta$-summability means from $H_{A}^{p(\cdot)}\left(\mathbb{R}^{n}\right)$ to $L^{p(\cdot)}\left(\mathbb{R}^{n}\right)$, obtained in [75], are presented.

Section 5 is devoted to the introduction of the real-variable theory of anisotropic variable Hardy-Lorentz spaces $H_{A}^{p(\cdot), q}\left(\mathbb{R}^{n}\right)$, with $p(\cdot)$ as in Section 4 and $q \in(0, \infty]$, which are the generalizations of the spaces $H_{A}^{p, q}\left(\mathbb{R}^{n}\right)$ considered in Section 3. To this end, we successively recall the notions of variable Lorentz spaces $L^{p(\cdot), q}\left(\mathbb{R}^{n}\right)$ from [59] and the spaces $H_{A}^{p(\cdot), q}\left(\mathbb{R}^{n}\right)$ from [78]. Then various real-variable characterizations of the spaces $H_{A}^{p(\cdot), q}\left(\mathbb{R}^{n}\right)$, respectively, in terms of maximal functions, atoms, finite atoms, and the Lusin area functions as well as the Littlewood-Paley $g$-functions or $g_{\lambda}^{*}$-functions, established in $[76,78]$, are presented. Some errors and gaps existing in the proof of the sufficiency of [78, Theorem 5.2] are also corrected and sealed in Subsection 5.2. As an application of the real-variable characterizations of both $H_{A}^{p(\cdot)}\left(\mathbb{R}^{n}\right)$ and $H_{A}^{p(\cdot), q}\left(\mathbb{R}^{n}\right)$, a real interpolation result, obtained in [78, Theorem 6.2], is displayed. Moreover, we review a criterion on the boundedness of some sublinear operators from $H_{A}^{p(\cdot), q}\left(\mathbb{R}^{n}\right)$ into a quasiBanach space and the boundedness of maximal operators of the $\theta$-summability means from $H_{A}^{p(\cdot), q}\left(\mathbb{R}^{n}\right)$ to $L^{p(\cdot), q}\left(\mathbb{R}^{n}\right)$; see [76].

In Section 6, we present the real-variable theory of anisotropic Musielak-Orlicz Hardy spaces $H_{A}^{\varphi}\left(\mathbb{R}^{n}\right)$ with $\varphi: \mathbb{R}^{n} \times[0, \infty) \rightarrow[0, \infty)$ being an anisotropic growth function (see [68, Definition 3]). For this purpose, we first recall the notions of the class of uniform anisotropic Muckenhoupt weights, anisotropic growth functions as well as MusielakOrlicz spaces $L^{\varphi}\left(\mathbb{R}^{n}\right)$, and then the definition of the spaces $H_{A}^{\varphi}\left(\mathbb{R}^{n}\right)$. Moreover, we point out that the anisotropic Musielak-Orlicz Hardy space $H_{A}^{\varphi}\left(\mathbb{R}^{n}\right)$ and the anisotropic vari- 
able Hardy space $H_{A}^{p(\cdot)}\left(\mathbb{R}^{n}\right)$ (see Section 4) cannot cover each other; see Remark 6.1(ii) below. Then we display several real-variable characterizations of the spaces $H_{A}^{\varphi}\left(\mathbb{R}^{n}\right)$, respectively, by means of atoms, molecules and the Lusin area functions as well as the Littlewood-Paley $g$-functions or $g_{\lambda}^{*}$-functions, which are established in $[67,73,74]$. Some errors and gaps existing in the proof of the sufficiency of [67, Theorem 2.8] are also corrected and sealed in Subsection 6.3. As an application of these real-variable characterizations, the boundedness of integral anisotropic Calderón-Zygmund operators from $H_{A}^{\varphi}\left(\mathbb{R}^{n}\right)$ to itself (or to the Musielak-Orlicz space $L^{\varphi}\left(\mathbb{R}^{n}\right)$ ), the dual spaces of $H_{A}^{\varphi}\left(\mathbb{R}^{n}\right)$ and the characterizations of $H_{A}^{\varphi}\left(\mathbb{R}^{n}\right)$ via the so-called tight frame multiwavelets, obtained in $[73,74]$, are reviewed.

\section{Notions and notation}

In this section, we give some notation and recall some notions on expansive matrices and homogeneous quasi-norms which are used throughout this article.

We always let $\mathbb{N}:=\{1, \cdots\}, \mathbb{Z}_{+}:=\{0\} \cup \mathbb{N}$ and $\overrightarrow{0}_{n}$ be the origin of $\mathbb{R}^{n}$. For any multi-index $\alpha:=\left(\alpha_{1}, \cdots, \alpha_{n}\right) \in\left(\mathbb{Z}_{+}\right)^{n}=: \mathbb{Z}_{+}^{n}$, let $|\alpha|:=\alpha_{1}+\cdots+\alpha_{n}$ and

$$
\partial^{\alpha}:=\left(\frac{\partial}{\partial x_{1}}\right)^{\alpha_{1}} \cdots\left(\frac{\partial}{\partial x_{n}}\right)^{\alpha_{n}} .
$$

We use the symbol $C$ to denote a positive constant which is independent of the main parameters and may change from line to line, and use $C_{(\alpha, \beta, \cdots)}$ to denote a positive constant depending on the indicated parameters $\alpha, \beta, \cdots$. The symbol $f \lesssim g$ means $f \leq C g$ and, when $f \lesssim g \lesssim f$, we write $f \sim g$. We also use the following convention: If $f \leq C g$ and $g=h$ or $g \leq h$, we then write $f \lesssim g \sim h$ or $f \lesssim g \lesssim h$, rather than $f \lesssim g=h$ or $f \lesssim g \leq h$. In addition, for any set $F \subset \mathbb{R}^{n}$, we denote by $\mathbf{1}_{F}$ its characteristic function and by $|F|$ its $n$-dimensional Lebesgue measure. For any $r \in[1, \infty]$, we denote by $r^{\prime}$ its conjugate index, namely, $1 / r+1 / r^{\prime}=1$ and by $\lfloor s\rfloor$ (resp. $\lceil s\rceil$ ) the largest (resp. least) integer not greater (resp. less) than $s$ for any $s \in \mathbb{R}$.

Denote by $L_{\text {loc }}^{1}\left(\mathbb{R}^{n}\right)$ the set of all locally integrable functions on $\mathbb{R}^{n}$. For any $r \in$ $(0, \infty]$ and measurable set $E \subset \mathbb{R}^{n}$, denote by the symbol $L^{r}(E)$ the set of all measurable functions $f$ such that, when $r \in(0, \infty)$,

$$
\|f\|_{L^{r}(E)}:=\left[\int_{E}|f(x)|^{r} d x\right]^{1 / r}<\infty
$$

and

$$
\|f\|_{L^{\infty}(E)}:=\underset{x \in E}{\operatorname{ess} \sup }|f(x)|<\infty .
$$


Recall that an $n \times n$ matrix $A$ is called an expansive matrix, shortly a dilation, if

$$
\min _{\lambda \in \sigma(A)}|\lambda|>1,
$$

here and thereafter, $\sigma(A)$ denotes the set of all eigenvalues of $A$ (see, for instance, [12]).

The following notion of homogeneous quasi-norms is just [12, p. 6, Definition 2.3].

Definition 2.1. Let $A$ be a given dilation. A measurable mapping $\rho: \mathbb{R}^{n} \rightarrow[0, \infty)$ is called a homogeneous quasi-norm, associated with $A$, if

(i) $x \neq \overrightarrow{0}_{n}$ implies $\rho(x) \in(0, \infty)$;

(ii) for any $x \in \mathbb{R}^{n}, \rho(A x)=b \rho(x)$, here and thereafter, $b:=|\operatorname{det} A|$;

(iii) there exists a constant $R \in[1, \infty)$ such that, for any $x, y \in \mathbb{R}^{n}, \rho(x+y) \leq R[\rho(x)+$ $\rho(y)]$.

By [12, p. 5, Lemma 2.2], we know that, for a given dilation $A$, there exist some $r \in$ $(1, \infty)$ and an open ellipsoid $\Delta$, with $|\Delta|=1$, such that $\Delta \subset r \Delta \subset A \Delta$. Then, for any $i \in \mathbb{Z}$, it is easy to see that $B_{i}:=A^{i} \Delta$ is open, $B_{i} \subset r B_{i} \subset B_{i+1}$ and $\left|B_{i}\right|=b^{i}$. For any $x \in \mathbb{R}^{n}$ and $i \in \mathbb{Z}, x+B_{i}$ is called a dilated ball. Let

$$
\mathfrak{B}:=\left\{x+B_{i}: x \in \mathbb{R}^{n} \text { and } i \in \mathbb{Z}\right\},
$$

and

$$
\tau:=\inf \left\{i \in \mathbb{Z}: r^{i} \geq 2\right\} .
$$

For any given dilation $A$, due to [12, p. 6, Lemma 2.4], we can use the step homogeneous quasi-norm $\rho$ defined by setting, for any $x \in \mathbb{R}^{n}$,

$$
\rho(x):=\sum_{i \in \mathbb{Z}} b^{i} \mathbf{1}_{B_{i+1} \backslash B_{i}}(x) \quad \text { when } x \neq \overrightarrow{0}_{n}, \quad \text { or else } \rho\left(\overrightarrow{0}_{n}\right):=0,
$$

for convenience. Let $\lambda_{-}$and $\lambda_{+}$be two positive numbers such that

$$
1<\lambda_{-} \leq \min \{|\lambda|: \lambda \in \sigma(A)\} \leq \max \{|\lambda|: \lambda \in \sigma(A)\} \leq \lambda_{+} .
$$

In particular, when $A$ is diagonalizable over $\mathbb{C}$, we can let

$$
\lambda_{-}:=\min \{|\lambda|: \lambda \in \sigma(A)\} \quad \text { and } \quad \lambda_{+}:=\max \{|\lambda|: \lambda \in \sigma(A)\} .
$$

Otherwise, we can choose them sufficiently close to these equalities in accordance with what we need in our arguments.

Recall also that a Schwartz function is an infinitely differentiable function $\phi$ satisfying, for any $\ell \in \mathbb{Z}_{+}$and multi-index $\alpha \in \mathbb{Z}_{+}^{n}$,

$$
\|\phi\|_{\alpha, \ell}:=\sup _{x \in \mathbb{R}^{n}}[\rho(x)]^{\ell}\left|\partial^{\alpha} \phi(x)\right|<\infty .
$$


Let $\mathcal{S}\left(\mathbb{R}^{n}\right)$ be the set of all Schwartz functions as above, equipped with the topology determined by $\left\{\|\cdot\|_{\alpha, \ell}\right\}_{\alpha \in \mathbb{Z}_{+}^{n}, \ell \in \mathbb{Z}_{+}}$, and $\mathcal{S}^{\prime}\left(\mathbb{R}^{n}\right)$ its dual space, equipped with the weak-* topology. For any $N \in \mathbb{Z}_{+}$, let

$\mathcal{S}_{N}\left(\mathbb{R}^{n}\right):=\left\{\phi \in \mathcal{S}\left(\mathbb{R}^{n}\right):\|\phi\|_{\mathcal{S}_{N}\left(\mathbb{R}^{n}\right)}:=\sup _{\substack{\alpha \in \mathbb{Z}_{+}^{n} \\|\alpha| \leq N}} \sup _{x \in \mathbb{R}^{n}}\left[\left|\partial^{\alpha} \phi(x)\right| \max \left\{1,[\rho(x)]^{N}\right\}\right] \leq 1\right\}$.

Throughout this article, for any $\phi \in \mathcal{S}\left(\mathbb{R}^{n}\right)$ and $i \in \mathbb{Z}$, let $\phi_{i}(\cdot):=b^{-i} \phi\left(A^{-i} \cdot\right)$.

\section{Anisotropic Hardy-Lorentz spaces}

In this section, we first recall the definition of anisotropic Hardy-Lorentz spaces $H_{A}^{p, q}\left(\mathbb{R}^{n}\right)$ introduced in [77] and then present various real-variable characterizations of $H_{A}^{p, q}\left(\mathbb{R}^{n}\right)$ established recently in $[77,79]$.

\subsection{Definition of anisotropic Hardy-Lorentz spaces}

We begin with the notion of Lorentz spaces. Let $p \in(0, \infty)$ and $q \in(0, \infty]$. The Lorentz space $L^{p, q}\left(\mathbb{R}^{n}\right)$ is defined to be the set of all measurable functions $f$ with finite $L^{p, q}\left(\mathbb{R}^{n}\right)$ quasi-norms $\|f\|_{L^{p, q}\left(\mathbb{R}^{n}\right)}$ given by

$$
\|f\|_{L^{p, q\left(\mathbb{R}^{n}\right)}}:= \begin{cases}\left\{\frac{q}{p} \int_{0}^{\infty}\left[t^{1 / p} f^{*}(t)\right]^{q} \frac{d t}{t}\right\}^{1 / q}, & \text { when } q \in(0, \infty), \\ \sup _{t \in(0, \infty)}\left[t^{1 / p} f^{*}(t)\right], & \text { when } q=\infty,\end{cases}
$$

where $f^{*}$ denotes the non-increasing rearrangement of $f$, namely, for any $t \in(0, \infty)$

$$
f^{*}(t):=\inf \left\{\alpha \in(0, \infty): d_{f}(\alpha) \leq t\right\} .
$$

Here and thereafter, for any $\alpha \in(0, \infty)$,

$$
d_{f}(\alpha):=\left|\left\{x \in \mathbb{R}^{n}:|f(x)|>\alpha\right\}\right| .
$$

Then, when $q \in(0, \infty)$,

$$
\|f\|_{L^{p, q}\left(\mathbb{R}^{n}\right)} \sim\left\{\int_{0}^{\infty} \alpha^{q-1}\left[d_{f}(\alpha)\right]^{q / p} d \alpha\right\}^{1 / q} \sim\left\{\sum_{k \in \mathbb{Z}}\left[2^{k}\left\{d_{f}\left(2^{k}\right)\right\}^{1 / p}\right]^{q}\right\}^{1 / q}
$$

and

$$
\|f\|_{L^{p, \infty}\left(\mathbb{R}^{n}\right)} \sim \sup _{\alpha \in(0, \infty)}\left\{\alpha\left[d_{f}(\alpha)\right]^{1 / p}\right\} \sim \sup _{k \in \mathbb{Z}}\left\{2^{k}\left[d_{f}\left(2^{k}\right)\right]^{1 / p}\right\},
$$

where the positive equivalence constants are independent of $f$; see [46]. 
Definition 3.1. Let $\varphi \in \mathcal{S}\left(\mathbb{R}^{n}\right)$ and $f \in \mathcal{S}^{\prime}\left(\mathbb{R}^{n}\right)$. The non-tangential maximal function $M_{\varphi}(f)$ with respect to $\varphi$ is defined by setting, for any $x \in \mathbb{R}^{n}$,

$$
M_{\varphi}(f)(x):=\sup _{y \in x+B_{k}, k \in \mathbb{Z}}\left|f * \varphi_{k}(y)\right| .
$$

Moreover, for any given $N \in \mathbb{N}$, the non-tangential grand maximal function $M_{N}(f)$ of $f \in \mathcal{S}^{\prime}\left(\mathbb{R}^{n}\right)$ is defined by setting, for any $x \in \mathbb{R}^{n}$,

$$
M_{N}(f)(x):=\sup _{\varphi \in \mathcal{S}_{N}\left(\mathbb{R}^{n}\right)} M_{\varphi}(f)(x) .
$$

The following notion of anisotropic Hardy-Lorentz spaces is just [77, Definition 2.5].

Definition 3.2. Let $p \in(0, \infty), q \in(0, \infty]$ and

$$
N_{(p)}:= \begin{cases}\left\lfloor\left(\frac{1}{p}-1\right) \frac{\ln b}{\ln \lambda_{-}}\right\rfloor+2, & \text { when } p \in(0,1], \\ 2, & \text { when } p \in(1, \infty) .\end{cases}
$$

For any $N \in \mathbb{N} \cap\left[N_{(p)}, \infty\right)$, the anisotropic Hardy-Lorentz space $H_{A}^{p, q}\left(\mathbb{R}^{n}\right)$ is defined by setting

$$
H_{A}^{p, q}\left(\mathbb{R}^{n}\right):=\left\{f \in \mathcal{S}^{\prime}\left(\mathbb{R}^{n}\right): M_{N}(f) \in L^{p, q}\left(\mathbb{R}^{n}\right)\right\}
$$

and, for any $f \in H_{A}^{p, q}\left(\mathbb{R}^{n}\right)$, let $\|f\|_{H_{A}^{p, q}\left(\mathbb{R}^{n}\right)}:=\left\|M_{N}(f)\right\|_{L^{p, q}\left(\mathbb{R}^{n}\right)}$.

Remark 3.1. (i) Even though the quasi-norm of $H_{A}^{p, q}\left(\mathbb{R}^{n}\right)$ in Definition 3.2 depends on $N$, from [77, Theorem 3.6] (see also Theorem 3.1 below), it follows that the space $H_{A}^{p, q}\left(\mathbb{R}^{n}\right)$ is independent of the choice of $N$ as long as $N \in \mathbb{N} \cap\left[N_{(p)}, \infty\right)$.

(ii) Obviously, when $p=q, H_{A}^{p, q}\left(\mathbb{R}^{n}\right)$ becomes the anisotropic Hardy space $H_{A}^{p}\left(\mathbb{R}^{n}\right)$ introduced by Bownik in [12] and, when $q=\infty, H_{A}^{p, q}\left(\mathbb{R}^{n}\right)$ is the anisotropic weak Hardy space $H_{A}^{p, \infty}\left(\mathbb{R}^{n}\right)$ investigated by Ding and Lan in [27].

(iii) Very recently, via the variable Lorentz spaces $\mathcal{L}^{p(\cdot), q(\cdot)}\left(\mathbb{R}^{n}\right)$ in [31], where

$$
p(\cdot), q(\cdot):(0, \infty) \rightarrow(0, \infty)
$$

are bounded measurable functions, Almeida et al. [2] investigated the anisotropic variable Hardy-Lorentz spaces $H^{p(\cdot), q(\cdot)}\left(\mathbb{R}^{n}, A\right)$. Clearly, when $p(\cdot) \equiv$ a constant $\epsilon$ $(0, \infty)$ and $q(\cdot) \equiv$ a constant $\in(0, \infty)$, the space $H^{p(\cdot), q(\cdot)}\left(\mathbb{R}^{n}, A\right)$ goes back to the anisotropic Hardy-Lorentz space $H_{A}^{p, q}\left(\mathbb{R}^{n}\right)$. However, the space $H^{p(\cdot), q(\cdot)}\left(\mathbb{R}^{n}, A\right)$ cannot cover the space $H_{A}^{p, \infty}\left(\mathbb{R}^{n}\right)$, because the variable exponent $q(\cdot)$ in $\mathcal{L}^{p(\cdot), q(\cdot)}\left(\mathbb{R}^{n}\right)$ belongs to $(0, \infty)$.

(iv) Let $p \in(0, \infty), q \in(0, \infty]$ and $N \in \mathbb{N} \cap\left[N_{(p)}, \infty\right)$. Then $H_{A}^{p, q}\left(\mathbb{R}^{n}\right) \subset \mathcal{S}^{\prime}\left(\mathbb{R}^{n}\right)$ with continuous inclusion and $H_{A}^{p, q}\left(\mathbb{R}^{n}\right)$ is complete; see [77, Propositions 2.7 and 2.8]. 


\subsection{Some equivalent characterizations of $H_{A}^{p, q}\left(\mathbb{R}^{n}\right)$}

Recall that the notion of anisotropic $(p, r, s)$-atoms was first introduced by Bownik in [12, p. 19, Definition 4.1].

Definition 3.3. An anisotropic triplet $(p, r, s)$ is said to be admissible if $p \in(0,1], r \in$ $(1, \infty]$ and

$$
s \in \mathbb{Z}_{+} \cap\left[\left\lfloor\left(\frac{1}{p}-1\right) \frac{\ln b}{\ln \lambda_{-}}\right\rfloor, \infty\right) .
$$

For an admissible anisotropic triplet $(p, r, s)$, a measurable function $a$ on $\mathbb{R}^{n}$ is called an anisotropic $(p, r, s)$-atom (shortly, a $(p, r, s)$-atom) if

(i) supp $a:=\left\{x \in \mathbb{R}^{n}: a(x) \neq 0\right\} \subset B$, where $B \in \mathfrak{B}$ and $\mathfrak{B}$ is as in (2.1);

(ii) $\|a\|_{L^{r}\left(\mathbb{R}^{n}\right)} \leq|B|^{1 / r-1 / p}$;

(iii) for any $\alpha \in \mathbb{Z}_{+}^{n}$ with $|\alpha| \leq s, \int_{\mathbb{R}^{n}} a(x) x^{\alpha} d x=0$.

Via $(p, r, s)$-atoms, Liu et al. [77] introduced the anisotropic atomic Hardy-Lorentz space $H_{A}^{p, r, r, q}\left(\mathbb{R}^{n}\right)$ as follows.

Definition 3.4. Let $A$ be a dilation, $(p, r, s)$ an admissible anisotropic triplet and $q \in$ $(0, \infty]$. The anisotropic atomic Hardy-Lorentz space $H_{A}^{p, r, s, q}\left(\mathbb{R}^{n}\right)$ is defined to be the set

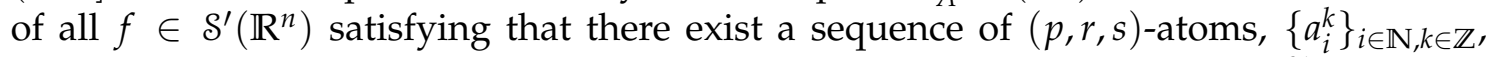
supported, respectively, in $\left\{B_{i}^{k}\right\}_{i \in \mathbb{N}, k \in \mathbb{Z}} \subset \mathfrak{B}$, and a positive constant $\widetilde{C}$ such that $\sum_{i \in \mathbb{N}} \mathbf{1}_{B_{i}^{k}}(x) \leq \widetilde{C}$ for any $x \in \mathbb{R}^{n}$ and $k \in \mathbb{Z}$, and

$$
f=\sum_{k \in \mathbb{Z}} \sum_{i \in \mathbb{N}} \lambda_{i}^{k} a_{i}^{k} \quad \text { in } \mathcal{S}^{\prime}\left(\mathbb{R}^{n}\right)
$$

where $\lambda_{i}^{k} \sim 2^{k}\left|B_{i}^{k}\right|^{1 / p}$ for any $k \in \mathbb{Z}$ and $i \in \mathbb{N}$ with the positive equivalence constants independent of $k$ and $i$.

Moreover, for any $f \in H_{A}^{p, r, s, q}\left(\mathbb{R}^{n}\right)$, let

$$
\|f\|_{H_{A}^{p, r, s, q}\left(\mathbb{R}^{n}\right)}:=\inf \left\{\left[\sum_{k \in \mathbb{Z}}\left(\sum_{i \in \mathbb{N}}\left|\lambda_{i}^{k}\right|^{p}\right)^{q / p}\right]^{1 / q}: f=\sum_{k \in \mathbb{Z}} \sum_{i \in \mathbb{N}} \lambda_{i}^{k} a_{i}^{k}\right\}
$$

with the usual modification made when $q=\infty$, where the infimum is taken over all the decompositions of $f$ as above.

We next recall the definition of anisotropic molecular Hardy-Lorentz spaces $H_{A}^{p, r, s, \varepsilon, q}\left(\mathbb{R}^{n}\right)$ from [77]. 
Definition 3.5. (i) An anisotropic quadruple $(p, r, s, \varepsilon)$ is said to be admissible if $p \in$ $(0,1], r \in(1, \infty], s$ is as in (3.5) and $\varepsilon \in(0, \infty)$. For an admissible anisotropic quadruple $(p, r, s, \varepsilon)$, a measurable function $m$ is called an anisotropic $(p, r, s, \varepsilon)$ molecule (shortly, a $(p, r, s, \varepsilon)$-molecule) associated with a dilated ball $B \in \mathfrak{B}$ if

(i) $)_{1}$ for each $j \in \mathbb{Z}_{+}$,

$$
\|m\|_{L^{r}\left(U_{j}(B)\right)} \leq b^{-j \varepsilon}|B|^{1 / r-1 / p},
$$

where $U_{0}(B):=B$ and, for any $j \in \mathbb{N}, U_{j}(B):=\left(A^{j} B\right) \backslash\left(A^{j-1} B\right) ;$

(i) $)_{2}$ for any $\alpha \in \mathbb{Z}_{+}^{n}$ with $|\alpha| \leq s$,

$$
\int_{\mathbb{R}^{n}} m(x) x^{\alpha} d x=0
$$

(ii) Let $(p, r, s, \varepsilon)$ be an admissible anisotropic quadruple and $q \in(0, \infty]$. The anisotropic molecular Hardy-Lorentz space $H_{A}^{p, r, s, \varepsilon, q}\left(\mathbb{R}^{n}\right)$ is defined to be the set of all $f \in \mathcal{S}^{\prime}\left(\mathbb{R}^{n}\right)$ satisfying that there exist a sequence of $(p, r, s, \varepsilon)$-molecules,

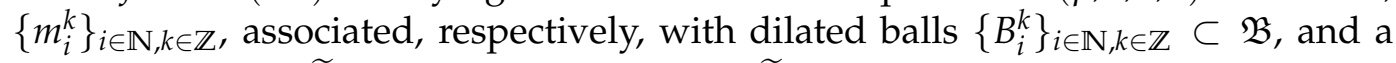
positive constant $\widetilde{C}$ such that $\sum_{i \in \mathbb{N}} \mathbf{1}_{B_{i}^{k}}(x) \leq \widetilde{C}$ for any $k \in \mathbb{Z}$ and $x \in \mathbb{R}^{n}$, and

$$
f=\sum_{k \in \mathbb{Z}} \sum_{i \in \mathbb{N}} \lambda_{i}^{k} m_{i}^{k} \quad \text { in } \mathcal{S}^{\prime}\left(\mathbb{R}^{n}\right),
$$

where $\lambda_{i}^{k} \sim 2^{k}\left|B_{i}^{k}\right|^{1 / p}$ for any $k \in \mathbb{Z}$ and $i \in \mathbb{N}$. Moreover, for any $f \in H_{A}^{p, r, s, \varepsilon, q}\left(\mathbb{R}^{n}\right)$, let

$$
\|f\|_{H_{A}^{p, r, s, \varepsilon, q}\left(\mathbb{R}^{n}\right)}:=\inf \left\{\left[\sum_{k \in \mathbb{Z}}\left(\sum_{i \in \mathbb{N}}\left|\lambda_{i}^{k}\right|^{p}\right)^{q / p}\right]^{1 / q}: f=\sum_{k \in \mathbb{Z}} \sum_{i \in \mathbb{N}} \lambda_{i}^{k} m_{i}^{k}\right\}
$$

with the usual modification made when $q=\infty$, where the infimum is taken over all the decompositions of $f$ as above.

The following atomic and molecular characterizations are, respectively, from [77, Theorems 3.6 and 3.9].

Theorem 3.1. Let $(p, r, s, \varepsilon)$ be an admissible anisotropic quadruple as in Definition 3.5 with $\varepsilon \in\left(\max \left\{1,(s+1) \log _{b}\left(\lambda_{+}\right)\right\}, \infty\right), q \in(0, \infty]$ and $N \in \mathbb{N} \cap\left[N_{(p)}, \infty\right)$. Then

$$
H_{A}^{p, r, s, q}\left(\mathbb{R}^{n}\right)=H_{A}^{p, q}\left(\mathbb{R}^{n}\right)=H_{A}^{p, r, s, \varepsilon, q}\left(\mathbb{R}^{n}\right)
$$

with equivalent quasi-norms. 
Let $\varphi \in \mathcal{S}\left(\mathbb{R}^{n}\right)$. The radial maximal function $M_{\varphi}^{0}(f)$ of $f \in \mathcal{S}^{\prime}\left(\mathbb{R}^{n}\right)$, with respect to $\varphi$, is defined by setting, for any $x \in \mathbb{R}^{n}$,

$$
M_{\varphi}^{0}(f)(x):=\sup _{k \in \mathbb{Z}}\left|f * \varphi_{k}(x)\right| .
$$

In [77, Theorem 4.9], the authors also obtained the maximal function characterizations of $H_{A}^{p, q}\left(\mathbb{R}^{n}\right)$ as follows.

Theorem 3.2. Suppose that $p \in(0, \infty), q \in(0, \infty]$ and $\varphi \in \mathcal{S}\left(\mathbb{R}^{n}\right)$ satisfying $\int_{\mathbb{R}^{n}} \varphi(x) d x \neq 0$. Then, for any $f \in \mathcal{S}^{\prime}\left(\mathbb{R}^{n}\right)$, the following statements are mutually equivalent:

(i) $f \in H_{A}^{p, q}\left(\mathbb{R}^{n}\right)$;

(ii) $M_{\varphi}(f) \in L^{p, q}\left(\mathbb{R}^{n}\right)$;

(iii) $M_{\varphi}^{0}(f) \in L^{p, q}\left(\mathbb{R}^{n}\right)$.

Moreover, there exist two positive constants $C$ and $\widetilde{C}$, independent of $f$, such that

$$
\|f\|_{H_{A}^{p, q}\left(\mathbb{R}^{n}\right)} \leq C\left\|M_{\varphi}^{0}(f)\right\|_{L^{p, q\left(\mathbb{R}^{n}\right)}} \leq C\left\|M_{\varphi}(f)\right\|_{L^{p, q}\left(\mathbb{R}^{n}\right)} \leq \widetilde{C}\|f\|_{H_{A}^{p, q}\left(\mathbb{R}^{n}\right)} .
$$

Definition 3.6. For an admissible anisotropic triplet $(p, r, s), q \in(0, \infty]$, the anisotropic finite atomic Hardy-Lorentz space $H_{A, \text { fin }}^{p, r, q}\left(\mathbb{R}^{n}\right)$ is defined to be the set of all $f \in \mathcal{S}^{\prime}\left(\mathbb{R}^{n}\right)$ sat-

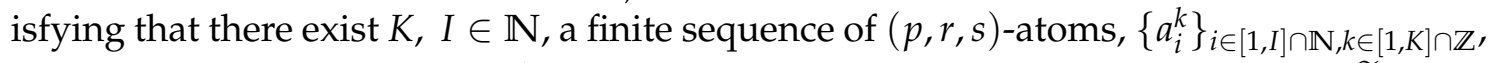
supported, respectively, in $\left\{B_{i}^{k}\right\}_{i \in[1, I] \cap \mathbb{N}, k \in[1, K] \cap \mathbb{Z}} \subset \mathfrak{B}$, and a positive constant $\widetilde{C}$, independent of $I$ and $K$, such that $\sum_{i=1}^{I} \mathbf{1}_{B_{i}^{k}}(x) \leq \widetilde{C}$ for any $x \in \mathbb{R}^{n}$ and $k \in[1, K] \cap \mathbb{Z}$, and

$$
f=\sum_{k=1}^{K} \sum_{i=1}^{I} \lambda_{i}^{k} a_{i}^{k} \quad \text { in } \mathcal{S}^{\prime}\left(\mathbb{R}^{n}\right)
$$

where $\lambda_{i}^{k} \sim 2^{k}\left|B_{i}^{k}\right|^{1 / p}$ for any $k \in[1, K] \cap \mathbb{Z}$ and $i \in[1, I] \cap \mathbb{N}$ with the positive equivalence constants independent of $k, K$ and $i$, I. Moreover, for any $f \in H_{A, \text { fin }}^{p, r, s, q}\left(\mathbb{R}^{n}\right)$, let

$$
\|f\|_{H_{A, f i n}^{p, r, q}\left(\mathbb{R}^{n}\right)}:=\inf \left\{\left[\sum_{k=1}^{K}\left(\sum_{i=1}^{I}\left|\lambda_{i}^{k}\right|^{p}\right)^{\frac{q}{p}}\right]^{\frac{1}{q}}: f=\sum_{k=1}^{K} \sum_{i=1}^{I} \lambda_{i}^{k} a_{i}^{k}, \quad K, I \in \mathbb{N}\right\}
$$

with the usual modification made when $q=\infty$, where the infimum is taken over all the decompositions of $f$ as above.

In what follows, denote by $C\left(\mathbb{R}^{n}\right)$ the set of all continuous functions. The following conclusion was established in [77, Theorem 5.7].

Theorem 3.3. Let $(p, r, s)$ be an admissible anisotropic triplet and $q \in(0, \infty]$.

(i) If $r \in(1, \infty)$, then $\|\cdot\|_{H_{A, \text { fin }}^{p, r, s, q}\left(\mathbb{R}^{n}\right)}$ and $\|\cdot\|_{H_{A}^{p, q}\left(\mathbb{R}^{n}\right)}$ are equivalent quasi-norms on $H_{A, \text { fin }}^{p, r, s, q}\left(\mathbb{R}^{n}\right)$;

(ii) $\|\cdot\|_{H_{A, \text { fin }}^{p, \infty, q}\left(\mathbb{R}^{n}\right)}$ and $\|\cdot\|_{H_{A}^{p, q}\left(\mathbb{R}^{n}\right)}$ are equivalent quasi-norms on $H_{A, \text { fin }}^{p, \infty, s, q}\left(\mathbb{R}^{n}\right) \cap C\left(\mathbb{R}^{n}\right)$. 


\subsection{Littlewood-Paley function characterizations of $H_{A}^{p, q}\left(\mathbb{R}^{n}\right)$}

In this subsection, we display the characterizations of $H_{A}^{p, q}\left(\mathbb{R}^{n}\right)$ in terms of the anisotropic Lusin area function, the anisotropic Littlewood-Paley $g$-function or $g_{\lambda}^{*}$-function; see [79]. Meanwhile, we also correct some errors and seal some gaps existing in the proof of the sufficiency of [79, Theorem 2.7], namely, the Lusin area function characterizations of $H_{A}^{p, q}\left(\mathbb{R}^{n}\right)$.

Recall that a distribution $f \in \mathcal{S}^{\prime}\left(\mathbb{R}^{n}\right)$ is said to vanish weakly at infinity if, for each $\psi \in \mathcal{S}\left(\mathbb{R}^{n}\right), f * \psi_{k} \rightarrow 0$ in $\mathcal{S}^{\prime}\left(\mathbb{R}^{n}\right)$ as $k \rightarrow \infty$. Denote by $\mathcal{S}_{0}^{\prime}\left(\mathbb{R}^{n}\right)$ the set of all $f \in \mathcal{S}^{\prime}\left(\mathbb{R}^{n}\right)$ vanishing weakly at infinity. Let $\imath:=\sqrt{-1}$ and, for any $x:=\left(x_{1}, \cdots, x_{n}\right)$, $v:=\left(v_{1}, \cdots, v_{n}\right) \in \mathbb{R}^{n}, x \cdot v:=\sum_{k=1}^{n} x_{k} v_{k}$. For any $f \in L^{1}\left(\mathbb{R}^{n}\right)$, denote by $\widehat{f}$ the Fourier transform of $f$, which is defined by setting, for any $v \in \mathbb{R}^{n}$,

$$
\widehat{f}(v):=\int_{\mathbb{R}^{n}} f(x) e^{-2 \pi \imath x \cdot v} d x .
$$

The following Calderón reproducing formula is just [17, Proposition 2.14]. In what follows, $C_{c}^{\infty}\left(\mathbb{R}^{n}\right)$ denotes the set of all infinitely differentiable functions with compact support on $\mathbb{R}^{n}$.

Lemma 3.1. Let $s \in \mathbb{Z}_{+}$and $A:=\left(a_{i, j}\right)_{1 \leq i, j \leq n}$ be a dilation. Assume that $\theta \in C_{c}^{\infty}\left(\mathbb{R}^{n}\right)$ satisfies $\operatorname{supp} \theta \subset B_{0}$,

$$
\int_{\mathbb{R}^{n}} x^{\gamma} \theta(x) d x=0 \quad \text { for any } \gamma \in \mathbb{Z}_{+}^{n} \text { with }|\gamma| \leq s,
$$

and $\widehat{\theta}(\xi) \geq C$ for any $\xi \in\left\{x \in \mathbb{R}^{n}:(2\|A\|)^{-1} \leq \rho(x) \leq 1\right\}$, where $C$ is a positive constant and

$$
\|A\|:=\left(\sum_{i, j=1}^{n}\left|a_{i, j}\right|^{2}\right)^{1 / 2} .
$$

Then there exists some $\psi \in \mathcal{S}\left(\mathbb{R}^{n}\right)$ such that

(i) supp $\widehat{\psi}$ is compact and away from the origin;

(ii) for any $\xi \in \mathbb{R}^{n} \backslash\left\{\overrightarrow{0}_{n}\right\}$,

$$
\sum_{j \in \mathbb{Z}} \widehat{\psi}\left(\left(A^{*}\right)^{j} \xi\right) \widehat{\theta}\left(\left(A^{*}\right)^{j} \xi\right)=1,
$$

where $A^{*}$ denotes the adjoint matrix of $A$.

Moreover, for any $f \in \mathcal{S}_{0}^{\prime}\left(\mathbb{R}^{n}\right), f=\sum_{j \in \mathbb{Z}} f * \psi_{j} * \theta_{j}$ in $\mathcal{S}^{\prime}\left(\mathbb{R}^{n}\right)$.

Let $\theta \in \mathcal{S}\left(\mathbb{R}^{n}\right)$ be as in Lemma 3.1 with $s$ as in (3.5). For any $f \in \mathcal{S}^{\prime}\left(\mathbb{R}^{n}\right)$ and $\lambda \in(0, \infty)$, the anisotropic Lusin area function $S(f)$, the anisotropic Littlewood-Paley $g$-function 
$g(f)$ and the anisotropic Littlewood-Paley $g_{\lambda}^{*}$-function $g_{\lambda}^{*}(f)$ are defined, respectively, by setting, for any $x \in \mathbb{R}^{n}$,

$$
\begin{aligned}
& S(f)(x):=\left[\sum_{k \in \mathbb{Z}} b^{-k} \int_{x+B_{k}}\left|f * \theta_{k}(y)\right|^{2} d y\right]^{1 / 2}, \\
& g(f)(x):=\left[\sum_{k \in \mathbb{Z}}\left|f * \theta_{k}(x)\right|^{2}\right]^{1 / 2},
\end{aligned}
$$

and

$$
g_{\lambda}^{*}(f)(x):=\left\{\sum_{k \in \mathbb{Z}} b^{-k} \int_{\mathbb{R}^{n}}\left[\frac{b^{k}}{b^{k}+\rho(x-y)}\right]^{\lambda}\left|f * \theta_{k}(y)\right|^{2} d y\right\}^{1 / 2} .
$$

In [79, Theorems 2.7 through 2.9], the authors characterized the space $H_{A}^{p, q}\left(\mathbb{R}^{n}\right)$, respectively, in terms of the anisotropic Lusin area function, the anisotropic Littlewood-Paley $g$-function or $g_{\lambda}^{*}$-function as follows.

Theorem 3.4. Let $p \in(0,1]$ and $q \in(0, \infty]$. Then

(i) $f \in H_{A}^{p, q}\left(\mathbb{R}^{n}\right)$ if and only if $f \in \mathcal{S}_{0}^{\prime}\left(\mathbb{R}^{n}\right)$ and $S(f) \in L^{p, q}\left(\mathbb{R}^{n}\right)$. Moreover, there exists a positive constant $C$ such that, for any $f \in H_{A}^{p, q}\left(\mathbb{R}^{n}\right)$,

$$
\frac{1}{C}\|S(f)\|_{L^{p, q}\left(\mathbb{R}^{n}\right)} \leq\|f\|_{H_{A}^{p, q}\left(\mathbb{R}^{n}\right)} \leq C\|S(f)\|_{L^{p, q}\left(\mathbb{R}^{n}\right)} .
$$

(ii) The conclusion as in (i) remains true if $S(f)$ is replaced, respectively, by $g(f)$ or $g_{\lambda}^{*}(f)$ with $\lambda \in(2 / p, \infty)$.

Remark 3.2. Let $S_{\theta}(f)$ and $S_{\psi}(f)$ be the anisotropic Lusin area functions defined, respectively, by using $\theta$ and $\psi$ as in Lemma 3.1. We should point out that, in the original proof of the sufficiency of Theorem 3.4(i) (namely, [79, Theorem 2.7]), the authors used both $S_{\theta}(f)$ and $S_{\psi}(f)$; see, respectively, the proofs of [79, (3.23) and (3.29)]. Thus, the following fact is needed: for any $f \in \mathcal{S}_{0}^{\prime}\left(\mathbb{R}^{n}\right)$, the $L^{p, q}\left(\mathbb{R}^{n}\right)$ quasi-norms of the anisotropic Lusin area function $S(f)$ are independent of the choices of $\theta$ and $\psi$ as in Lemma 3.1. However, in the original proof of the sufficiency of [79, Theorem 2.7], the authors did not present the proof of this necessary fact. To seal this gap, we first present the following conclusions.

Theorem 3.5. Let $p \in(0,1], q \in(0, \infty]$ and $\theta, \psi$ be as in Lemma 3.1 with $s$ as in (3.5). Then there exists a positive constant $C$ such that, for any $f \in \mathcal{S}_{0}^{\prime}\left(\mathbb{R}^{n}\right)$,

$$
\frac{1}{C}\left\|S_{\theta}(f)\right\|_{L^{p, q}\left(\mathbb{R}^{n}\right)} \leq\left\|S_{\psi}(f)\right\|_{L^{p, q}\left(\mathbb{R}^{n}\right)} \leq C\left\|S_{\theta}(f)\right\|_{L^{p, q}\left(\mathbb{R}^{n}\right)},
$$

where $S_{\theta}(f)$ and $S_{\psi}(f)$ are the anisotropic Lusin area functions as in (3.8) defined, respectively, via $\theta$ and $\psi$. 
To prove Theorem 3.5, we first recall the notion of anisotropic Hardy-Littlewood maximal operators as follows.

Definition 3.7. The anisotropic Hardy-Littlewood maximal operator $M_{\mathrm{HL}}$ is defined by setting, for any $f \in L_{\text {loc }}^{1}\left(\mathbb{R}^{n}\right)$ and $x \in \mathbb{R}^{n}$,

$$
M_{\mathrm{HL}}(f)(x):=\sup _{k \in \mathbb{Z}} \sup _{y \in x+B_{k}} \frac{1}{\left|B_{k}\right|} \int_{y+B_{k}}|f(z)| d z=\sup _{x \in B \in \mathfrak{B}} \frac{1}{|B|} \int_{B}|f(z)| d z,
$$

where $\mathfrak{B}$ is as in (2.1).

The following Fefferman-Stein vector-valued inequality of the maximal operator $M_{\mathrm{HL}}$ on the Lorentz space $L^{p, q}\left(\mathbb{R}^{n}\right)$ was obtained in [79, Lemma 4.5(i)], which plays a key role in the proof of Theorem 3.5.

Lemma 3.2. Let $p \in(1, \infty), q \in(0, \infty]$ and $r \in(1, \infty]$. Then there exists a positive constant $C$ such that, for any sequence $\left\{f_{j}\right\}_{j}$ of measurable functions,

$$
\left\|\left\{\sum_{j}\left[M_{\mathrm{HL}}\left(f_{j}\right)\right]^{r}\right\}^{1 / r}\right\|_{L^{p, q}\left(\mathbb{R}^{n}\right)} \leq C\left\|\left[\sum_{j}\left|f_{j}\right|^{r}\right]^{1 / r}\right\|_{L^{p, q}\left(\mathbb{R}^{n}\right)} .
$$

We also need the following conclusion, which is just [17, Lemma 2.3] and originates from [21, Theorem 11].

Lemma 3.3. Let $A$ be a dilation. Then there exists a set

$$
\mathfrak{Q}:=\left\{Q_{\alpha}^{k} \subset \mathbb{R}^{n}: k \in \mathbb{Z}, \alpha \in E_{k}\right\}
$$

of open subsets, where $E_{k}$ is an index set, such that

(i) for each $k \in \mathbb{Z},\left|\mathbb{R}^{n} \backslash \bigcup_{\alpha} Q_{\alpha}^{k}\right|=0$ and, when $\alpha \neq \beta, Q_{\alpha}^{k} \cap Q_{\beta}^{k}=\varnothing$;

(ii) for any $\alpha, \beta, k$, $\ell$ with $\ell \geq k$, either $Q_{\alpha}^{k} \cap Q_{\beta}^{\ell}=\varnothing$ or $Q_{\alpha}^{\ell} \subset Q_{\beta}^{k}$;

(iii) for each $(\ell, \beta)$ and each $k<\ell$, there exists a unique $\alpha$ such that $Q_{\beta}^{\ell} \subset Q_{\alpha}^{k}$;

(iv) there exist some $v \in \mathbb{Z} \backslash \mathbb{Z}_{+}$and $u \in \mathbb{N}$ such that, for any $Q_{\alpha}^{k}$ with $k \in \mathbb{Z}$ and $\alpha \in E_{k}$, there exists an $x_{Q_{\alpha}^{k}} \in Q_{\alpha}^{k}$ such that, for any $x \in Q_{\alpha}^{k}$,

$$
x_{Q_{\alpha}^{k}}+B_{v k-u} \subset Q_{\alpha}^{k} \subset x+B_{v k+u} .
$$

Henceforth, we call $\mathfrak{Q}:=\left\{Q_{\alpha}^{k}\right\}_{k \in \mathbb{Z}, \alpha \in E_{k}}$ from Lemma 3.3 dyadic cubes and $k$ the level, denoted by $\ell\left(Q_{\alpha}^{k}\right)$, of the dyadic cube $Q_{\alpha}^{k}$ for any $k \in \mathbb{Z}$ and $\alpha \in E_{k}$.

The following technical lemma is necessary, which is just [51, Lemma 6.14]. In what follows, for any $t \in \mathbb{R}$, we denote by $\lceil t\rceil$ the least integer not less than $t$. 
Lemma 3.4. Let $s$ be as in (3.5), $v$ and $u$ as in Lemma 3.3(iv) and

$$
r \in\left(\frac{\ln b}{\ln b+(s+1) \ln \lambda_{-}}, 1\right] .
$$

Then there exists a positive constant $C$ such that, for any $k, i \in \mathbb{Z},\left\{c_{Q}\right\}_{Q \in \mathfrak{Q}} \subset[0, \infty)$ with $\mathfrak{Q}$ as in Lemma 3.3, and $x \in \mathbb{R}^{n}$,

$$
\begin{gathered}
\sum_{\substack{\ell(Q)=\left[\frac{k-u}{v}\right\rceil i f k \geq u \\
\ell(Q)=\left[\frac{k-u}{v}\right] i f k<u}}|Q| \frac{b^{(k \vee i)(s+1) \frac{\ln \lambda_{-}}{\ln b}}}{\left[b^{(k \vee i)}+\rho\left(x-z_{Q}\right)\right]^{(s+1) \frac{\ln \lambda_{-}}{\ln b}+1}} c_{Q} \\
\leq C b^{-[k-(k \vee i)](1 / r-1)}\left\{M_{\mathrm{HL}}\left(\sum_{\begin{array}{l}
\ell(Q)=\left[\frac{k-u}{v}\right\rceil i f k \geq u \\
\ell(Q)=\left[\frac{k-u}{v}\right] i f k<u
\end{array}}\left[c_{Q}\right]^{r} \mathbf{1}_{Q}\right)(x)\right\}^{1 / r},
\end{gathered}
$$

where $\ell(Q)$ denotes the level of $Q \in \mathfrak{Q}, z_{Q} \in Q$ and, for any $k, i \in \mathbb{Z}, k \vee i:=\max \{k, i\}$.

We now prove Theorem 3.5.

Proof of Theorem 3.5. By symmetry, to show this theorem, we only need to prove that, for any $f \in \mathcal{S}_{0}^{\prime}\left(\mathbb{R}^{n}\right)$,

$$
\left\|S_{\theta}(f)\right\|_{L^{p, q}\left(\mathbb{R}^{n}\right)} \lesssim\left\|S_{\psi}(f)\right\|_{L^{p, q}\left(\mathbb{R}^{n}\right)} .
$$

To this end, for any $i \in \mathbb{Z}, x \in \mathbb{R}^{n}$ and $y \in x+B_{i}$, let

$$
I_{\theta}^{(i)}(f)(y):=f * \theta_{i}(y) .
$$

Then, by Lemma 3.1 and the Lebesgue dominated convergence theorem, we find that, for any $i \in \mathbb{Z}, x \in \mathbb{R}^{n}$ and $y \in x+B_{i}$,

$$
\begin{aligned}
I_{\theta}^{(i)}(f)(y) & =\sum_{k \in \mathbb{Z}} f * \psi_{k} * \theta_{k} * \theta_{i}(y)=\sum_{k \in \mathbb{Z}} \int_{\mathbb{R}^{n}} f * \psi_{k}(z) \theta_{k} * \theta_{i}(y-z) d z \\
& =\sum_{k \in \mathbb{Z}} \sum_{\substack{\ell(Q)=\left[\frac{k-u}{v} \\
\ell(Q)=\left\lfloor\frac{k-u}{v}\right] i f k \geq u\right.}} \int_{Q} f * \psi_{k}(z) \theta_{k} * \theta_{i}(y-z) d z
\end{aligned}
$$

in $\mathcal{S}^{\prime}\left(\mathbb{R}^{n}\right)$, where all the symbols are the same as those used in Lemma 3.4.

On another hand, by [17, Lemma 5.4], we know that, for any $k, i \in \mathbb{Z}$ and $x \in \mathbb{R}^{n}$,

$$
\left|\theta_{k} * \theta_{i}(x)\right| \lesssim b^{-(s+1)|k-i| \frac{\ln \lambda_{-}}{\ln b}} \frac{b^{(k \vee i)(s+1) \frac{\ln \lambda_{-}}{\ln b}}}{\left[b^{(k \vee i)}+\rho(x)\right]^{(s+1) \frac{\ln \lambda_{-}+1}{\ln b}}} .
$$


This further implies that, for any $Q \in \mathfrak{Q}$ with

$$
\ell(Q)= \begin{cases}\left\lceil\frac{k-u}{v}\right\rceil, & \text { when } k \in[u, \infty) \cap \mathbb{Z}, \\ \left\lfloor\frac{k-u}{v}\right\rfloor, & \text { when } k \in(-\infty, u) \cap \mathbb{Z},\end{cases}
$$

there exists some $z_{Q} \in Q$ such that, for any $k, i \in \mathbb{Z}, x \in \mathbb{R}^{n}, y \in x+B_{i}$ and $z \in Q$,

$$
\left|\theta_{k} * \theta_{i}(y-z)\right| \lesssim b^{-(s+1)|k-i| \frac{\ln \lambda_{-}}{\ln b}} \frac{b^{(k \vee i)(s+1) \frac{\ln \lambda_{-}}{\ln b}}}{\left[b^{(k \vee i)}+\rho\left(x-z_{Q}\right)\right]^{(s+1) \frac{\ln \lambda_{-}}{\ln b}+1}} .
$$

Moreover, for any $Q \in \mathfrak{Q}$ satisfying (3.12), we have $B_{v \ell(Q)+u} \subset B_{k}$. From this, the Hölder inequality and Lemma 3.3(iv), we deduce that, for any $z \in Q$,

$$
\begin{aligned}
\frac{1}{|Q|}\left|\int_{Q} f * \psi_{k}(y) d y\right| & \leq\left[\frac{1}{|Q|} \int_{Q}\left|f * \psi_{k}(y)\right|^{2} d y\right]^{1 / 2} \\
& \leq\left[\frac{1}{\left|B_{v \ell(Q)-u}\right|} \int_{z+B_{v \ell(Q)+u}}\left|f * \psi_{k}(y)\right|^{2} d y\right]^{1 / 2} \\
& \lesssim\left[b^{-k} \int_{z+B_{k}}\left|f * \psi_{k}(y)\right|^{2} d y\right]^{1 / 2} \sim \mathrm{Y}_{\psi}^{(k)}(f)(z)
\end{aligned}
$$

where, for any $k \in \mathbb{Z}$ and $z \in \mathbb{R}^{n}$,

$$
\mathrm{Y}_{\psi}^{(k)}(f)(z):=\left[b^{-k} \int_{z+B_{k}}\left|f * \psi_{k}(y)\right|^{2} d y\right]^{1 / 2}
$$

Thus, for any $k \in \mathbb{Z}$ and $Q \in \mathfrak{Q}$ satisfying (3.12),

$$
\frac{1}{|Q|}\left|\int_{Q} f * \psi_{k}(y) d y\right| \lesssim \inf _{z \in Q} Y_{\psi}^{(k)}(f)(z)
$$

By this, (3.11), (3.13) and Lemma 3.4, we conclude that, for any given $r \in\left(\frac{\ln b}{\ln b+(s+1) \ln \lambda_{-}}, 1\right]$ and for any $i \in \mathbb{Z}, x \in \mathbb{R}^{n}$ and $y \in x+B_{i}$,

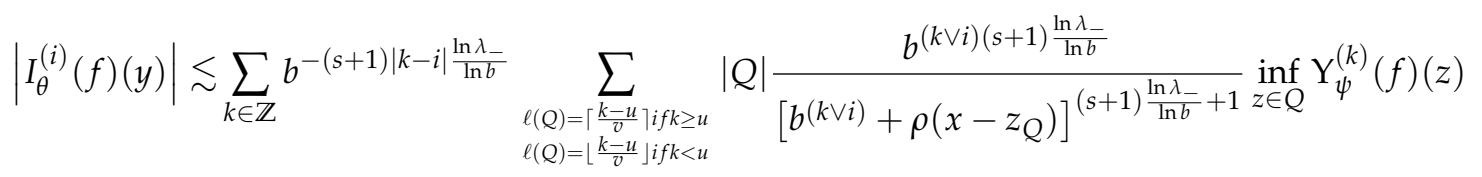

$$
\begin{aligned}
& \lesssim \sum_{k \in \mathbb{Z}} b^{-(s+1)|k-i| \frac{\ln \lambda_{-}}{\ln b} b^{-[k-(k \vee i)](1 / r-1)}} \\
& \times\left\{M_{\mathrm{HL}}\left(\sum_{\substack{\ell(Q)=\left[\frac{k-u}{v}\right] i f k \geq u \\
\ell(Q)=\left[\frac{k-u}{v}\right] i f k<u}} \inf _{z \in Q}\left[\mathrm{Y}_{\psi}^{(k)}(f)(z)\right]^{r} \mathbf{1}_{\mathrm{Q}}\right)(x)\right\}^{1 / r} \\
& =: J_{(r, i)}(x) \text {. }
\end{aligned}
$$


By (3.5), we can choose some $r \in\left(\frac{\ln b}{\ln b+(s+1) \ln \lambda_{-}}, p\right)$. Therefore, from (3.14), it follows that, for any $x \in \mathbb{R}^{n}$,

$$
\left[S_{\theta}(f)(x)\right]^{2}=\sum_{i \in \mathbb{Z}} b^{-i} \int_{x+B_{i}}\left|I_{\theta}^{(i)}(f)(y)\right|^{2} d y \lesssim \sum_{i \in \mathbb{Z}}\left[J_{(r, i)}(x)\right]^{2} .
$$

This, together with the Hölder inequality and the fact that $r>\frac{\ln b}{\ln b+(s+1) \ln \lambda_{-}}$, implies that, for any $x \in \mathbb{R}^{n}$,

$$
\begin{aligned}
& {\left[S_{\theta}(f)(x)\right]^{2} \lesssim \sum_{i \in \mathbb{Z}} \sum_{k \in \mathbb{Z}} b^{-\left.(s+1)|k-i|\right|^{\ln \lambda_{-}} \ln b} b^{-[k-(k \vee i)](1 / r-1)}} \\
& \times\left\{M_{\mathrm{HL}}\left(\sum_{\substack{\ell(Q)=\left\lceil\frac{k-u}{k}\right\rceil i f k \geq u \\
\ell(Q)=\left\lfloor\frac{-u}{v} \backslash i f k<u\right.}} \inf _{z \in Q}\left[\mathrm{Y}_{\psi}^{(k)}(f)(z)\right]^{r} \mathbf{1}_{Q}\right)(x)\right\}^{2 / r} \\
& \lesssim \sum_{k \in \mathbb{Z}}\left\{M_{\mathrm{HL}}\left(\sum_{\substack{\ell(Q)=\left[\frac{k-u}{v}\right] i f k \geq u \\
\ell(Q)=\left[\frac{k-u}{v}\right] i f k<u}} \inf _{z \in Q}\left[\mathrm{Y}_{\psi}^{(k)}(f)(z)\right]^{r} \mathbf{1}_{Q}\right)(x)\right\}^{2 / r} \\
& \lesssim \sum_{k \in \mathbb{Z}}\left\{M_{\mathrm{HL}}\left(\left[\mathrm{Y}_{\psi}^{(k)}(f)\right]^{r}\right)(x)\right\}^{2 / r} \text {. }
\end{aligned}
$$

Thus, by the fact that $r<p$ and Lemma 3.2, we find that

$$
\begin{aligned}
\left\|S_{\theta}(f)\right\|_{L^{p, q}\left(\mathbb{R}^{n}\right)} & \lesssim\left\|\left(\sum_{k \in \mathbb{Z}}\left\{M_{\mathrm{HL}}\left(\left[\mathrm{Y}_{\psi}^{(k)}(f)\right]^{r}\right)(x)\right\}^{2 / r}\right)^{r / 2}\right\|_{L^{p / r, q / r}\left(\mathbb{R}^{n}\right)}^{1 / r} \\
& \lesssim\left\|\left(\sum_{k \in \mathbb{Z}}\left[\mathrm{Y}_{\psi}^{(k)}(f)\right]^{2}\right)^{1 / 2}\right\|_{L^{p, q}\left(\mathbb{R}^{n}\right)} \sim\left\|S_{\psi}(f)\right\|_{L^{p, q}\left(\mathbb{R}^{n}\right)},
\end{aligned}
$$

which implies (3.10) holds true and hence completes the proof of Theorem 3.5.

Recall that, for any given $N \in \mathbb{N}$, the radial grand maximal function $M_{N}^{0}(f)$ of $f \in$ $\mathcal{S}^{\prime}\left(\mathbb{R}^{n}\right)$ is defined by setting, for any $x \in \mathbb{R}^{n}$,

$$
M_{N}^{0}(f)(x):=\sup _{\varphi \in \mathcal{S}_{N}\left(\mathbb{R}^{n}\right)} M_{\varphi}^{0}(f)(x)
$$

with $M_{\varphi}^{0}(f)$ as in (3.6).

Finally, we give out the proof of the sufficiency of Theorem 3.4(i). 
Proof of the sufficiency of Theorem 3.4(i). Let $\psi$ be as in Lemma 3.1, $f \in \mathcal{S}_{0}^{\prime}\left(\mathbb{R}^{n}\right)$ and $S(f) \in$ $L^{p, q}\left(\mathbb{R}^{n}\right)$. Then, by Theorem 3.5, we know that $S_{\psi}(f) \in L^{p, q}\left(\mathbb{R}^{n}\right)$. Thus, we only need to show that $f \in H_{A}^{p, q}\left(\mathbb{R}^{n}\right)$ and

$$
\|f\|_{H_{A}^{p, q}\left(\mathbb{R}^{n}\right)} \lesssim\left\|S_{\psi}(f)\right\|_{L^{p, q}\left(\mathbb{R}^{n}\right)} .
$$

To this end, for any $k \in \mathbb{Z}$, let $\Omega_{k}:=\left\{x \in \mathbb{R}^{n}: S_{\psi}(f)(x)>2^{k}\right\}$ and

$$
\mathfrak{Q}_{k}:=\left\{Q \in \mathfrak{Q}:\left|Q \cap \Omega_{k}\right|>\frac{|Q|}{2} \text { and }\left|Q \cap \Omega_{k+1}\right| \leq \frac{|Q|}{2}\right\} .
$$

Obviously, for any $Q \in \mathfrak{Q}$, there exists a unique $k \in \mathbb{Z}$ such that $Q \in \mathfrak{Q}_{k}$. Let $\left\{Q_{i}^{k}\right\}_{i}$ be the set of all maximal dyadic cubes in $\mathfrak{Q}_{k}$, namely, there exists no $Q \in \mathfrak{Q}_{k}$ such that $Q_{i}^{k} \varsubsetneqq Q$ for any $i$.

For any $Q \in \mathfrak{Q}$, let

$$
\widehat{Q}:=\left\{(y, t) \in \mathbb{R}_{+}^{n+1}:=\mathbb{R}^{n} \times(0, \infty): y \in Q, t \sim b^{v \ell(Q)+u}\right\},
$$

here and thereafter, $t \sim b^{v \ell(Q)+u}$ always means

$$
b^{v \ell(Q)+u+\tau} \leq t<b^{v[\ell(Q)-1]+u+\tau},
$$

where $u, v$ are as in Lemma 3.3(iv) and $\ell(Q)$ denotes the level of $Q$. Observe that, in the above inequality (3.18), $v$ is negative. Clearly, $\{\widehat{Q}\}_{Q \in \mathfrak{Q}}$ are mutually disjoint and

$$
\mathbb{R}_{+}^{n+1}=\bigcup_{k \in \mathbb{Z}} \bigcup_{i} B_{k, i}
$$

where, for any $k \in \mathbb{Z}$ and $i, B_{k, i}:=\bigcup_{Q \subset Q_{i}^{k}, Q \in \mathfrak{Q}_{k}} \widehat{Q}$. Then, by Lemma 3.3(ii) and (3.17), we easily find that $\left\{B_{k, i}\right\}_{k \in \mathbb{Z}, i}$ are also mutually disjoint.

Let $\psi$ and $\theta$ be as in Lemma 3.1 with $s$ as in (3.5). Then $\theta$ has the vanishing moments up to order s. From Lemma 3.1, the properties of the tempered distributions (see, for instance, [46, Theorem 2.3.20]) and (3.19), we deduce that, for any $f \in \mathcal{S}_{0}^{\prime}\left(\mathbb{R}^{n}\right)$ such that $S_{\psi}(f) \in L^{p, q}\left(\mathbb{R}^{n}\right)$, and for any $x \in \mathbb{R}^{n}$,

$$
f(x)=\sum_{k \in \mathbb{Z}} f * \psi_{k} * \theta_{k}(x)=\int_{\mathbb{R}_{+}^{n+1}} f * \psi_{t}(y) * \theta_{t}(x-y) d y d m(t) \quad \text { in } \mathcal{S}^{\prime}\left(\mathbb{R}^{n}\right),
$$

here and thereafter, $m(t)$ denotes the counting measure on $\mathbb{R}$, namely, for any set $E \subset \mathbb{R}$, $m(E)$ is the number of integers contained in $E$ if $E$ has only finitely many elements, or else $m(E):=\infty$. For each $k \in \mathbb{Z}, i$ and $x \in \mathbb{R}^{n}$, let

$$
h_{i}^{k}(x):=\int_{B_{k, i}} f * \psi_{t}(y) * \theta_{t}(x-y) d y d m(t) .
$$


Next we prove the sufficiency of Theorem 3.4(i) in three steps.

Step 1) The aim of this step is to show that

$$
\sum_{k \in \mathbb{Z}} \sum_{i} h_{i}^{k} \text { converges in } \mathcal{S}^{\prime}\left(\mathbb{R}^{n}\right)
$$

For this purpose, we claim that, for any given $r \in(1, \infty)$,

(i) for any $k \in \mathbb{Z}$, $i$ and $x \in \mathbb{R}^{n}$,

$$
h_{i}^{k}(x)=\sum_{Q \subset Q_{i}^{k}, Q \in \mathfrak{Q}_{k}} \int_{\widehat{Q}} f * \psi_{t}(y) \theta_{t}(x-y) d y d m(t)=: \sum_{Q \subset Q_{i}^{k}, Q \in \mathfrak{Q}_{k}} e_{Q}(x)
$$

holds true in $L^{r}\left(\mathbb{R}^{n}\right)$ and hence also in $\mathcal{S}^{\prime}\left(\mathbb{R}^{n}\right)$;

(ii) for any $k \in \mathbb{Z}$ and $i, h_{i}^{k}$ is a multiple of a $(p, r, s)$-atom.

Indeed, from $[79,(3.23)]$, it follows that, for any $x \in \mathbb{R}^{n}$,

$$
\left[S_{\theta}\left(\sum_{Q \subset Q_{i}^{k}, Q \in \mathfrak{Q}_{k}} e_{Q}\right)(x)\right]^{2} \lesssim \sum_{Q \subset Q_{i}^{k}, Q \in \mathfrak{Q}_{k}}\left[M_{\mathrm{HL}}\left(c_{Q} \mathbf{1}_{Q}\right)(x)\right]^{2}
$$

where $M_{\mathrm{HL}}$ denotes the Hardy-Littlewood maximal operator as in (3.9) and, for any $Q \subset Q_{i}^{k}$ and $Q \in \mathfrak{Q}_{k}$,

$$
c_{Q}:=\left[\int_{\widehat{Q}}\left|\psi_{t} * f(y)\right|^{2} d y \frac{d m(t)}{t}\right]^{1 / 2} .
$$

We first show assertion (i). To this end, for any $k \in \mathbb{Z}$, let

$$
\widehat{\Omega}_{k}:=\left\{x \in \mathbb{R}^{n}: M_{\mathrm{HL}}\left(\mathbf{1}_{\Omega_{k}}\right)(x)>\frac{1}{2} b^{-2 u}\right\}
$$

with $u$ as in Lemma 3.3(iv). Then, by an argument similar to that used in the estimation of $[79,(3.26)]$, we conclude that, for any given $r \in(1, \infty)$ and for any $k \in \mathbb{Z}$ and $i$,

$$
\left\|\sum_{Q \subset Q_{i}^{k}, Q \in \mathfrak{Q}_{k}} e_{Q}\right\|_{L^{r}\left(\mathbb{R}^{n}\right)} \lesssim\left\|\left[\sum_{Q \subset Q_{i}^{k}, Q \in \mathfrak{Q}_{k}}\left(c_{Q}\right)^{2} \mathbf{1}_{\mathrm{Q} \cap\left(\widehat{\Omega}_{k} \backslash \Omega_{k+1}\right)}\right]^{1 / 2}\right\|_{L^{r}\left(\mathbb{R}^{n}\right)} .
$$

On another hand, for any $k \in \mathbb{Z}, Q \in \mathfrak{Q}_{k}, x \in Q$ and $(y, t) \in \widehat{Q}$, by Lemma 3.3(iv) and $[12$, p. $8,(2.11)]$, we have

$$
x-y \in B_{v \ell(Q)+u}+B_{v \ell(Q)+u} \subset B_{v \ell(Q)+u+\tau}
$$


which, combined with (3.18) and the disjointness of $\{\widehat{Q}\}_{Q \subset Q_{i}^{k}}$, further implies that, for any $x \in \mathbb{R}^{n}$,

$$
\begin{aligned}
& \sum_{Q \subset Q_{i}^{k}, Q \in \mathfrak{Q}_{k}}\left(c_{Q}\right)^{2} \mathbf{1}_{Q \cap\left(\widehat{\Omega}_{k} \backslash \Omega_{k+1}\right)}(x) \\
= & \sum_{Q \subset Q_{i}^{k}, Q \in \mathfrak{Q}_{k}} \int_{\widehat{Q}}\left|\psi_{t} * f(y)\right|^{2} d y \frac{d m(t)}{t} \mathbf{1}_{Q \cap\left(\widehat{\Omega}_{k} \backslash \Omega_{k+1}\right)}(x) \\
\lesssim & {\left[S_{\psi}(f)(x)\right]^{2} \mathbf{1}_{Q_{i}^{k} \cap\left(\widehat{\Omega}_{k} \backslash \Omega_{k+1}\right)}(x) . }
\end{aligned}
$$

By this and Lemma 3.3(iv) again, we find that, for any given $r \in(1, \infty)$ and for any $k \in \mathbb{Z}$ and $i$,

$$
\begin{aligned}
&\left\|\left\{\sum_{Q \subset Q_{i}^{k}, Q \in \mathfrak{Q}_{k}}\left(c_{Q}\right)^{2} \mathbf{1}_{Q \cap\left(\widehat{\Omega}_{k} \backslash \Omega_{k+1}\right)}\right\}^{1 / 2}\right\|_{L^{r}\left(\mathbb{R}^{n}\right)}^{r} \\
& \leq \int_{\mathbb{R}^{n}}\left[S_{\psi}(f)(x)\right]^{r} \mathbf{1}_{Q_{i}^{k} \cap\left(\widehat{\Omega}_{k} \backslash \Omega_{k+1}\right)}(x) d x \\
& \lesssim 2^{k r}\left|Q_{i}^{k}\right| \lesssim 2^{k r} b^{v k+u}<\infty .
\end{aligned}
$$

For any $k \in \mathbb{Z}$ and $N \in \mathbb{N}$, let $\mathfrak{Q}_{k, N}:=\left\{Q \in \mathfrak{Q}_{k}:|\ell(Q)|>N\right\}$. Then, replacing $\sum_{Q \subset Q_{i}^{k}, Q \in \mathfrak{Q}_{k}} e_{Q}$ by $\sum_{Q \subset Q_{i}^{k}, Q \in \mathfrak{Q}_{k, N}} e_{Q}$ in (3.23), we know that, for any $k \in \mathbb{Z}, N \in \mathbb{N}$ and $i$,

$$
\left\|\sum_{Q \subset Q_{i}^{k}, Q \in \mathfrak{Q}_{k, N}} e_{Q}\right\|_{L^{r}\left(\mathbb{R}^{n}\right)} \lesssim\left\|\left[\sum_{Q \subset Q_{i}^{k}, Q \in \mathfrak{Q}_{k, N}}\left(c_{Q}\right)^{2} \mathbf{1}_{\mathrm{Q} \cap\left(\widehat{\Omega}_{k} \backslash \Omega_{k+1}\right)}\right]^{1 / 2}\right\|_{L^{r}\left(\mathbb{R}^{n}\right)} .
$$

From this, (3.24) and the Lebesgue dominated convergence theorem, we deduce that, for any given $r \in(1, \infty)$ and for any $k \in \mathbb{Z}$ and $i$,

$$
\left\|\sum_{Q \subset Q_{i}^{k}, Q \in \mathfrak{Q}_{k, N}} e_{Q}\right\|_{L^{r}\left(\mathbb{R}^{n}\right)} \rightarrow 0
$$

as $N \rightarrow \infty$, and hence

$$
\left\|\int_{\cup_{Q \subset Q_{i}^{k}, Q \in \mathfrak{Z}_{k, N}} \widehat{Q}} f * \psi_{t}(y) \theta_{t}(x-y) d y d m(t)\right\|_{L^{r}\left(\mathbb{R}^{n}\right)} \rightarrow 0
$$

as $N \rightarrow \infty$. Therefore, $h_{i}^{k}=\sum_{Q \subset Q_{i}^{k}, Q \in \mathfrak{Q}_{k}} e_{Q}$ in $L^{r}\left(\mathbb{R}^{n}\right)$. This finishes the proof of the above assertion (i). 
We now show assertion (ii). For this purpose, by assertion (i), we find that, for any $x \in \operatorname{supp} h_{i}^{k}, h_{i}^{k}(x) \neq 0$ implies that there exists some $Q \subset Q_{i}^{k}$ and $Q \in \mathfrak{Q}_{k}$ such that $e_{Q}(x) \neq 0$ and hence there exists a $(y, t) \in \widehat{Q}$ such that $x-y \in B_{v[\ell(Q)-1]+u+\tau}$. By this, Lemma 3.3(iv) and [12, p. 8, (2.11)], we have, for any $x \in \operatorname{supp} e_{Q}$,

$$
x \in y+B_{v[\ell(Q)-1]+u+\tau} \subset x_{Q}+B_{v \ell(Q)+u}+B_{v[\ell(Q)-1]+u+\tau} \subset x_{Q}+B_{v[\ell(Q)-1]+u+2 \tau} .
$$

Therefore, we obtain

$$
\text { supp } e_{Q} \subset x_{Q}+B_{v[\ell(Q)-1]+u+2 \tau} .
$$

From this, the fact that $h_{i}^{k}=\sum_{Q \subset Q_{i}^{k}, Q \in \mathfrak{Q}_{k}} e_{Q}$, (ii) and (iv) of Lemma 3.3 and [12, p. 8, (2.11)] again, it follows that

$$
\begin{aligned}
\operatorname{supp} h_{i}^{k} & \subset \bigcup_{Q \subset Q_{i}^{k}, Q \in \mathfrak{Q}_{k}}\left(x_{Q}+B_{v[\ell(Q)-1]+u+2 \tau}\right) \\
& \subset x_{Q_{i}^{k}}+B_{v \ell\left(Q_{i}^{k}\right)+u}+B_{v\left[\ell\left(Q_{i}^{k}\right)-1\right]+u+2 \tau} \\
& \subset x_{Q_{i}^{k}}+B_{v\left[\ell\left(Q_{i}^{k}\right)-1\right]+u+3 \tau}=: B_{i}^{k} .
\end{aligned}
$$

On another hand, by assertion (i) again, (3.23), the estimation of (3.24) and Lemma 3.3(iv), we conclude that

$$
\begin{aligned}
\left\|h_{i}^{k}\right\|_{L^{r}\left(\mathbb{R}^{n}\right)} & \lesssim\left\{\int_{\mathbb{R}^{n}}\left[S_{\psi}(f)(x)\right]^{r} \mathbf{1}_{Q_{i}^{k} \cap\left(\widehat{\Omega}_{k} \backslash \Omega_{k+1}\right)}(x) d x\right\}^{1 / r} \\
& \lesssim 2^{k}\left|Q_{i}^{k}\right|^{1 / r} \leq C_{(r)} 2^{k}\left|B_{i}^{k}\right|^{1 / r},
\end{aligned}
$$

where $C_{(r)}$ is a positive constant independent of $f, k$ and $i$. In addition, recall that $\theta$ has the vanishing moments up to order $s \geq\left\lfloor(1 / p-1) \ln b / \ln \lambda_{-}\right\rfloor$and so does $e_{Q}$. For any $k \in \mathbb{Z}, i, \gamma \in \mathbb{Z}_{+}^{n}$ with $|\gamma| \leq s$ and $x \in \mathbb{R}^{n}$, let $g(x):=x^{\gamma} \mathbf{1}_{B_{i}^{k}}(x)$ with $B_{i}^{k}$ as in (3.25). Clearly, $g \in L^{r^{\prime}}\left(\mathbb{R}^{n}\right)$ with $r \in(1, \infty)$ satisfying $1 / r+1 / r^{\prime}=1$. Thus, by the fact that $\left(L^{r^{\prime}}\left(\mathbb{R}^{n}\right)\right)^{*}=L^{r}\left(\mathbb{R}^{n}\right)$, (3.25) and the inclusion

$$
\operatorname{supp} e_{Q} \subset x_{Q}+B_{v[\ell(Q)-1]+u+2 \tau} \subset B_{i}^{k},
$$

we further have

$$
\int_{\mathbb{R}^{n}} h_{i}^{k}(x) x^{\gamma} d x=\left\langle h_{i}^{k}, g\right\rangle=\sum_{Q \subset Q_{i}^{k}, Q \in \mathfrak{Q}_{k}}\left\langle e_{Q}, g\right\rangle=\sum_{Q \subset Q_{i}^{k}, Q \in \mathfrak{Q}_{k}} \int_{\mathbb{R}^{n}} e_{Q}(x) x^{\gamma} d x=0 .
$$

This, together with (3.25) and (3.26), implies that $h_{i}^{k}$ is a multiple of a $(p, r, s)$-atom supported in $B_{i}^{k}$, which completes the proof of the above assertion (ii).

Now we prove (3.21). For any $k \in \mathbb{Z}$ and $i$, let

$$
\lambda_{i}^{k}:=C_{(r)} 2^{k}\left|B_{i}^{k}\right|^{1 / p} \quad \text { and } \quad a_{i}^{k}:=\left(\lambda_{i}^{k}\right)^{-1} h_{i}^{k},
$$


where $C_{(r)}$ is as in (3.26). Then, for any $k \in \mathbb{Z}$ and $i, a_{i}^{k}$ is a $(p, r, s)$-atom. To show (3.21), we next consider two cases: $i \in \mathbb{N}$ and $i \in\{1, \cdots, I\}$ with some $I \in \mathbb{N}$.

Case 1) $i \in \mathbb{N}$. In this case, to prove (3.21), by (3.27) and [77, Propositions 2.7 and 2.8], it suffices to show that

$$
\lim _{l \rightarrow \infty}\left\|\sum_{l \leq|k| \leq m} \sum_{l \leq i \leq m} \lambda_{i}^{k} a_{i}^{k}\right\|_{H_{A}^{p, q}\left(\mathbb{R}^{n}\right)}=0 .
$$

To do this, from the fact that, for any $l, m \in \mathbb{N}, \sum_{l \leq|k| \leq m} \sum_{l \leq i \leq m} \lambda_{i}^{k} a_{i}^{k} \in H_{A}^{p, r, s, q}\left(\mathbb{R}^{n}\right)$, Theorem 3.1, the mutual disjointness of $\left\{Q_{i}^{k}\right\}_{i}$ for any fixed $k \in \mathbb{Z}$, and Lemma 3.3(iv), we deduce that

$$
\begin{aligned}
& \left\|\sum_{l \leq|k| \leq m} \sum_{l \leq i \leq m} \lambda_{i}^{k} a_{i}^{k}\right\|_{H_{A}^{p, q}\left(\mathbb{R}^{n}\right)} \\
\lesssim & {\left[\sum_{l \leq|k| \leq m}\left(\sum_{l \leq i \leq m}\left|\lambda_{i}^{k}\right|^{p}\right)^{q / p}\right]^{1 / q} \sim\left[\sum_{l \leq|k| \leq m}\left(\sum_{l \leq i \leq m} 2^{k p}\left|B_{i}^{k}\right|\right)^{q / p}\right]^{1 / q} } \\
\sim & {\left[\sum_{l \leq|k| \leq m} 2^{k q}\left(\sum_{l \leq i \leq m}\left|Q_{i}^{k}\right|\right)^{q / p}\right]^{1 / q} \lesssim\left[\sum_{l \leq|k| \leq m} 2^{k q}\left|\Omega_{k}\right|^{q / p}\right]^{1 / q} }
\end{aligned}
$$

with the usual modification made when $q=\infty$. This, combined with the fact that $S_{\psi}(f) \in$ $L^{p, q}\left(\mathbb{R}^{n}\right),(3.2)$ and the completeness of the space $L^{p, q}\left(\mathbb{R}^{n}\right)$, implies that (3.28) holds true and hence so does (3.21) for Case 1).

Case 2) $i \in\{1, \cdots, I\}$ with some $I \in \mathbb{N}$. In this case, to show (3.21), it suffices to prove that

$$
\lim _{l \rightarrow \infty}\left\|\sum_{l \leq|k| \leq m} \sum_{i=1}^{I} \lambda_{i}^{k} a_{i}^{k}\right\|_{H_{A}^{p, q}\left(\mathbb{R}^{n}\right)}=0 .
$$

Indeed, by a proof similar to that of (3.28), we easily find that (3.29) also holds true. This finishes the proof of Case 2) and hence of (3.21).

Step 2) In this step, we prove that

$$
f=\sum_{k \in \mathbb{Z}} \sum_{i} \lambda_{i}^{k} a_{i}^{k} \quad \text { in } \mathcal{S}^{\prime}\left(\mathbb{R}^{n}\right)
$$

To this end, for any $x \in \mathbb{R}^{n}$, let

$$
\widetilde{f}(x):=\sum_{k \in \mathbb{Z}} \sum_{i} h_{i}^{k}(x)=\sum_{k \in \mathbb{Z}} \sum_{i} \int_{B_{k, i}} f * \psi_{t}(y) \theta_{t}(x-y) d y d m(t) \quad \text { in } \mathcal{S}^{\prime}\left(\mathbb{R}^{n}\right),
$$


here and thereafter, for any $k \in \mathbb{Z}$ and $i, B_{k, i}$ is as in (3.19). Then, to show (3.30), we only need to prove that

$$
f=\widetilde{f} \quad \text { in } \mathcal{S}^{\prime}\left(\mathbb{R}^{n}\right)
$$

To do this, by the above assertion (i), (3.17) and (3.18), we know that, for any given $r \in$ $(1, \infty)$ and for any $k \in \mathbb{Z}, i$ and $x \in \mathbb{R}^{n}$,

$$
\begin{aligned}
& h_{i}^{k}(x)=\lim _{N \rightarrow \infty} \int_{0}^{\infty} \int_{\mathbb{R}^{n}} f * \psi_{t}(y) \theta_{t}(x-y) \mathbf{1}_{\substack{Q \subset Q_{i}^{k}, Q \in \mathfrak{Q}_{k} \\
|\ell(Q)| \leq N}} \widehat{Q}(y, t) d y d m(t) \\
& =\lim _{N \rightarrow \infty} \int_{\gamma(N)}^{\eta(N)} \int_{\mathbb{R}^{n}} f * \psi_{t}(y) \theta_{t}(x-y) \mathbf{1}_{B_{k, i}}(y, t) d y d m(t)
\end{aligned}
$$

holds true in $L^{r}\left(\mathbb{R}^{n}\right)$ and also in $\mathcal{S}^{\prime}\left(\mathbb{R}^{n}\right)$, where, for any $N \in \mathbb{N}, \gamma(N):=b^{v N+u+1}$ and $\eta(N):=b^{-v(N+1)+u+1}$ with $v$ and $u$ as in Lemma 3.3(iv). We next consider two cases: $i \in \mathbb{N}$ and $i \in\{1, \cdots, I\}$ with some $I \in \mathbb{N}$.

Case 1) $i \in \mathbb{N}$. To deal with this case, for any $M \in \mathbb{N}$ and $x \in \mathbb{R}^{n}$, let

$$
\widetilde{f}_{M}(x):=f(x)-\sum_{|k| \in \mathbb{Z}_{+} \cap[0, M]} \sum_{i=1}^{M} \int_{B_{k, i}} f * \psi_{t}(y) \theta_{t}(x-y) d y d m(t) .
$$

Then, by (3.19), (3.20) and (3.32), we conclude that, for any $M \in \mathbb{N}$ and $x \in \mathbb{R}^{n}$,

$$
\begin{aligned}
\widetilde{f}_{M}(x)= & \lim _{N \rightarrow \infty} \int_{\gamma(N)}^{\eta(N)} \int_{\mathbb{R}^{n}} f * \psi_{t}(y) \theta_{t}(x-y) \mathbf{1}_{\cup_{k \in \mathbb{Z}} \cup_{i \in \mathbb{N}} B_{k, i}}(y, t) d y d m(t) \\
& \quad-\lim _{N \rightarrow \infty} \int_{\gamma(N)}^{\eta(N)} \int_{\mathbb{R}^{n}} f * \psi_{t}(y) \theta_{t}(x-y) \mathbf{1}_{\cup_{|k| \in \mathbb{Z}_{+} \cap[0, M]} \cup_{i=1}^{M} B_{k, i}}(y, t) d y d m(t) \\
= & \lim _{N \rightarrow \infty} \int_{\gamma(N)}^{\eta(N)} \int_{\mathbb{R}^{n}} f * \psi_{t}(y) \theta_{t}(x-y) \mathbf{1}_{|k| \in \mathbb{N} \cap[M+1, \infty)} \cup_{i=M+1}^{\infty} B_{k, i}(y, t) d y d m(t)
\end{aligned}
$$

holds true in $\mathcal{S}^{\prime}\left(\mathbb{R}^{n}\right)$. Observe that $H_{A}^{p, q}\left(\mathbb{R}^{n}\right)$ is continuously embedded into $\mathcal{S}^{\prime}\left(\mathbb{R}^{n}\right)$ (see [77, Proposition 2.7]). Therefore, to show (3.31), it suffices to prove that

$$
\left\|\widetilde{f}_{M}\right\|_{H_{A}^{p, q}\left(\mathbb{R}^{n}\right)} \rightarrow 0 \text { as } M \rightarrow \infty .
$$

For this purpose, we borrow some ideas from the proof of the atomic characterizations of $H_{A}^{p, q}\left(\mathbb{R}^{n}\right)$ (see [77, Theorem 3.6]). Indeed, for any $\varepsilon \in(0,1), M \in \mathbb{N}$ and $x \in \mathbb{R}^{n}$, let

$$
\widetilde{f}_{M}^{(\varepsilon)}(x):=\int_{\mathcal{\varepsilon}}^{\alpha / \varepsilon} \int_{\mathbb{R}^{n}} f * \psi_{t}(y) \theta_{t}(x-y) \mathbf{1}_{\cup_{|k| \in \mathbb{N} \cap[M+1, \infty)} \cup_{i=M+1}^{\infty} B_{k, i}}(y, t) d y d m(t),
$$


here and thereafter, $\alpha:=b^{-v+2(u+1)}$. Then, from the Lebesgue dominated convergence theorem, it follows that, for any $\varepsilon \in(0,1), M \in \mathbb{N}$ and $x \in \mathbb{R}^{n}$,

$$
\begin{aligned}
\tilde{f}_{M}^{(\varepsilon)}(x) & =\sum_{|k| \in \mathbb{N} \cap[M+1, \infty)} \sum_{i \in \mathbb{N} \cap[M+1, \infty)} \int_{\varepsilon}^{\alpha / \varepsilon} \int_{\mathbb{R}^{n}} f * \psi_{t}(y) \theta_{t}(x-y) \mathbf{1}_{B_{k, i}}(y, t) d y d m(t) \\
& =: \sum_{|k| \in \mathbb{N} \cap[M+1, \infty)} \sum_{i \in \mathbb{N} \cap[M+1, \infty)} h_{k, i}^{(\varepsilon)}(x)
\end{aligned}
$$

in $\mathcal{S}^{\prime}\left(\mathbb{R}^{n}\right)$.

On another hand, for any $\varepsilon \in(0,1)$ and $Q \in \mathfrak{Q}$, let

$$
\widehat{Q}_{\varepsilon}:=\left\{(y, t) \in \mathbb{R}^{n} \times(\varepsilon, \alpha / \varepsilon): y \in Q \text { and } t \sim b^{v \ell(Q)+u}\right\}
$$

where $v$ and $u$ are as in Lemma 3.3(iv) and $\ell(Q)$ denotes the level of $Q$. Obviously, for any given $\varepsilon \in(0,1),\left\{\widehat{Q}_{\varepsilon}\right\}_{Q \in \mathfrak{Q}}$ are mutually disjoint and

$$
\mathbb{R}^{n} \times(\varepsilon, \alpha / \varepsilon)=\bigcup_{k \in \mathbb{Z}} \bigcup_{i \in \mathbb{N}} B_{k, i}^{(\varepsilon)}
$$

where, for any $k \in \mathbb{Z}$ and $i \in \mathbb{N}, B_{k, i}^{(\varepsilon)}:=\bigcup_{Q \subset Q_{i}^{k}, Q \in \mathfrak{Q}_{k}} \widehat{Q}_{\varepsilon}$. Then, by Lemma 3.3(ii), we easily know that, for any given $\varepsilon \in(0,1),\left\{B_{k, i}^{(\varepsilon)}\right\}_{k \in \mathbb{Z}, i \in \mathbb{N}}$ are also mutually disjoint. Moreover, by some arguments similar to these used in the proofs of the above assertions (i) and (ii) in Step 1) with some slight modifications, we conclude that, for any $\varepsilon \in(0,1), M \in \mathbb{N}$, $|k|, i \in \mathbb{N} \cap[M+1, \infty)$ and $x \in \mathbb{R}^{n}$,

$$
h_{k, i}^{(\varepsilon)}(x)=\sum_{Q \subset Q_{i}^{k}, Q \in \mathfrak{Q}_{k}} e_{Q}^{(\varepsilon)}(x) \quad \text { in } \mathcal{S}^{\prime}\left(\mathbb{R}^{n}\right),
$$

where, for any $Q \subset Q_{i}^{k}, Q \in \mathfrak{Q}_{k}$ and $x \in \mathbb{R}^{n}$,

$$
e_{Q}^{(\varepsilon)}(x):=\int_{\widehat{Q}_{\varepsilon}} f * \psi_{t}(y) \theta_{t}(x-y) d y d m(t)
$$

and, for any given $r \in(1, \infty), h_{k, i}^{(\varepsilon)}$ is a multiple of a $(p, r, s)$-atom, namely, there exist

$$
\left\{\lambda_{k, i}\right\}_{|k|, i \in \mathbb{N} \cap[M+1, \infty)} \subset \mathbb{C}
$$

and a sequence of $(p, r, s)$-atoms, $\left\{a_{k, i}^{(\varepsilon)}\right\}_{|k|, i \in \mathbb{N} \cap[M+1, \infty)}$, supported, respectively, in

$$
\left\{B_{k, i}\right\}_{|k|, i \in \mathbb{N} \cap[M+1, \infty)} \subset \mathfrak{B}
$$


such that, for any $|k|, i \in \mathbb{N} \cap[M+1, \infty), h_{k, i}^{(\varepsilon)}=\lambda_{k, i} a_{k, i}^{(\varepsilon)}$ and $\lambda_{k, i} \sim 2^{k}\left|B_{k, i}\right|^{1 / p}$, here, for any $|k|, i \in \mathbb{N} \cap[M+1, \infty), \lambda_{k, i}$ and $B_{k, i}$ are independent of $\varepsilon$. Thus, for any given $\varepsilon \in(0,1), M \in \mathbb{N}$ and $x \in \mathbb{R}^{n}$,

$$
\widetilde{f}_{M}^{(\varepsilon)}(x)=\sum_{|k| \in \mathbb{N} \cap[M+1, \infty)} \sum_{i \in \mathbb{N} \cap[M+1, \infty)} \lambda_{k, i} a_{k, i}^{(\varepsilon)}(x) \quad \text { in } \mathcal{S}^{\prime}\left(\mathbb{R}^{n}\right),
$$

and

$$
\left[\sum_{|k| \in \mathbb{N} \cap[M+1, \infty)}\left(\sum_{i \in \mathbb{N} \cap[M+1, \infty)}\left|\lambda_{k, i}\right|^{p}\right)^{q / p}\right]^{1 / q}<\infty
$$

with the usual modification made when $q=\infty$.

For any $M \in \mathbb{N},|k| \in \mathbb{N} \cap[M+1, \infty)$ and $p \in(0,1]$, let

$$
\mu_{M, k}:=\left(\sum_{i \in \mathbb{N} \cap[M+1, \infty)}\left|B_{k, i}\right|\right)^{1 / p} \text { and } \beta:=\left(\frac{\ln b}{\ln \lambda_{-}}+N_{(p)}-1\right) \frac{\ln \lambda_{-}}{\ln b}>\frac{1}{p^{\prime}}
$$

where $N_{(p)}$ is as in Definition 3.2. Then, for any $r \in(1, \infty)$, there exists a $\delta \in(1 / r, 1)$ such that $\frac{1}{\beta}<\delta p<1$. We now rewrite (3.35) as

$$
\widetilde{f}_{M}^{(\varepsilon)}(x)=\sum_{k \in \mathbb{Z}} \sum_{i \in \mathbb{N} \cap[M+1, \infty)} \lambda_{k, i} a_{k, i}^{(\varepsilon)}(x) \quad \text { in } \mathcal{S}^{\prime}\left(\mathbb{R}^{n}\right),
$$

where, for any $|k| \in \mathbb{Z}_{+} \cap[0, M]$ and $i \in \mathbb{N} \cap[M+1, \infty), \lambda_{k, i}=0$. In addition, let $\varepsilon:=\gamma(N)$ with $N \in \mathbb{N} \cap\left[\left\lfloor\frac{-u-1}{v}\right\rfloor+1, \infty\right)$. Then, by (3.33), we find that

$$
M_{N_{(p)}}^{0}\left(\widetilde{f}_{M}\right)=M_{N_{(p)}}^{0}\left(\lim _{N \rightarrow \infty} \widetilde{f}_{M}^{(\gamma(N))}\right) \leq \liminf _{N \rightarrow \infty} M_{N_{(p)}}^{0}\left(\widetilde{f}_{M}^{(\gamma(N))}\right)
$$

where $M_{N_{(p)}}^{0}$ is as in (3.15) with $N$ replaced by $N_{(p)}$. Notice that, for any fixed $k_{0} \in \mathbb{Z}$ and for any $x \in \mathbb{R}^{n}$,

$$
\begin{aligned}
& M_{N_{(p)}}^{0}\left(\widetilde{f}_{M}^{(\gamma(N))}\right)(x) \\
\leq & M_{N_{(p)}}^{0}\left(\sum_{k=-\infty}^{k_{0}-1} \sum_{i=M+1}^{\infty} \lambda_{k, i} a_{k, i}^{(\gamma(N))}\right)(x)+\sum_{k=k_{0}}^{\infty} \sum_{i=M+1}^{\infty}\left|\lambda_{k, i}\right| M_{N_{(p)}}^{0}\left(a_{k, i}^{(\gamma(N))}\right)(x) \\
= & : \phi_{k_{0}}(x)+w_{k_{0}}(x) .
\end{aligned}
$$

To prove (3.34), we now consider two cases: $q / p \in[1, \infty]$ and $q / p \in(0,1)$. 
Case 1.1) $q / p \in[1, \infty]$. In this case, to show the desired conclusion, by a calculation similar to that of $[77,(3.22)]$, we claim that

$$
2^{k_{0} p}\left[d_{\phi_{k_{0}}}\left(2^{k_{0}}\right)\right]^{\delta} \lesssim \sum_{k=-\infty}^{k_{0}-1}\left[2^{k}\left(\mu_{M, k}\right)^{\delta}\right]^{p} \quad \text { and } \quad 2^{k_{0} \delta p} d_{w_{k_{0}}}\left(2^{k_{0}}\right) \lesssim \sum_{k=k_{0}}^{\infty}\left[2^{k \delta} \mu_{M, k}\right]^{p},
$$

where $d_{\phi_{k_{0}}}$ and $d_{w_{k_{0}}}$ are as in (3.1) with $f$ replaced, respectively, by $\phi_{k_{0}}$ and $w_{k_{0}}$. Then, from [12, p. 17, Proposition 3.10], (3.37), [1, Lemma 1.2], the facts that $\delta \in(0, q / p)$ and $\left|B_{k, i}\right| \sim \frac{\left|\lambda_{k, i}\right|^{p}}{2^{k p}}$, we deduce that, for any $M \in \mathbb{N}$,

$$
\begin{aligned}
\left\|\widetilde{f}_{M}\right\|_{H_{A}^{p, q}\left(\mathbb{R}^{n}\right)} & =\left\|M_{N_{(p)}}^{0}\left(\widetilde{f}_{M}\right)\right\|_{L^{p, q}\left(\mathbb{R}^{n}\right)} \leq\left\|\liminf _{N \rightarrow \infty} M_{N_{(p)}}^{0}\left(\widetilde{f}_{M}^{(\gamma(N))}\right)\right\|_{L^{p, q}\left(\mathbb{R}^{n}\right)} \\
& \lesssim\left\|\left\{2^{k} \mu_{M, k}\right\}_{k \in \mathbb{Z}}\right\|_{\ell q} \lesssim\left[\sum_{k \in \mathbb{Z}}\left(\sum_{i \in \mathbb{N} \cap[M+1, \infty)}\left|\lambda_{k, i}\right|^{p}\right)^{q / p}\right]^{1 / q} \\
& \sim\left[\sum_{|k| \in \mathbb{N} \cap[M+1, \infty)}\left(\sum_{i \in \mathbb{N} \cap[M+1, \infty)}\left|\lambda_{k, i}\right|^{p}\right)^{q / p}\right]^{1 / q} .
\end{aligned}
$$

This, combined with (3.36), implies that (3.34) holds true.

Case 1.2) $q / p \in(0,1)$. In this case, similarly to [77, (3.23) and (3.26)], we conclude that, for any $M \in \mathbb{N}$,

$$
2^{k_{0} p}\left[d_{\phi_{k_{0}}}\left(2^{k_{0}}\right)\right]^{\delta} \lesssim\left[\sum_{k=-\infty}^{k_{0}-1} 2^{\frac{k p}{\delta}}\left(\mu_{M, k}\right)^{p}\right]^{\delta} \quad \text { and } \quad 2^{k_{0} \delta p} d_{w_{k_{0}}}\left(2^{k_{0}}\right) \lesssim \sum_{k=k_{0}}^{\infty}\left[2^{k \delta} \mu_{M, k}\right]^{p} .
$$

This further respectively implies that

$$
\begin{aligned}
& \sum_{k_{0} \in \mathbb{Z}} 2^{k_{0} q}\left|\left\{x \in \mathbb{R}^{n}: \phi_{k_{0}}(x)>2^{k_{0}}\right\}\right|^{q / p} \\
\lesssim & \sum_{k_{0} \in \mathbb{Z}} 2^{k_{0}(q-q / \delta)} \sum_{k=-\infty}^{k_{0}-1} 2^{\frac{k q}{\delta}}\left(\mu_{M, k}\right)^{q} \\
\sim & \sum_{k \in \mathbb{Z}} \sum_{k_{0}=k+1}^{\infty} 2^{k_{0}(q-q / \delta)} 2^{\frac{k q}{\delta}}\left(\mu_{M, k}\right)^{q} \lesssim \sum_{k \in \mathbb{Z}} 2^{k q}\left(\mu_{M, k}\right)^{q},
\end{aligned}
$$

and

$$
\begin{aligned}
& \sum_{k_{0} \in \mathbb{Z}} 2^{k_{0} q}\left|\left\{x \in \mathbb{R}^{n}: w_{k_{0}}(x)>2^{k_{0}}\right\}\right|^{q / p} \\
\lesssim & \sum_{k_{0} \in \mathbb{Z}} 2^{k_{0} \widetilde{\delta} q} \sum_{k=k_{0}}^{\infty}\left[2^{k(1-\widetilde{\delta})} \mu_{M, k}\right]^{q}
\end{aligned}
$$




$$
\sim \sum_{k \in \mathbb{Z}}\left[2^{k(1-\widetilde{\delta})} \mu_{M, k}\right]^{q} \sum_{k_{0}=-\infty}^{k} 2^{k_{0} \tilde{\delta} q} \lesssim \sum_{k \in \mathbb{Z}} 2^{k q}\left(\mu_{M, k}\right)^{q}
$$

where $\widetilde{\delta}:=\frac{1-\delta}{2}$. From this, [12, p. 17, Proposition 3.10], (3.37), the facts that $\left|B_{k, i}\right| \sim \frac{\left|\lambda_{k, i}\right|^{p}}{2^{k p}}$ and $\mu_{M, k}:=\left(\sum_{i=M+1}^{\infty}\left|B_{k, i}\right|\right)^{1 / p}$, it follows that, for any $M \in \mathbb{N}$, (3.38) holds true, which, together with (3.36), implies that (3.34) also holds true in this case. This finishes the proof of (3.34) and hence of (3.30) for Case 1).

Case 2) $i \in\{1, \cdots, I\}$ with some $I \in \mathbb{N}$. For this case, repeating the proof of (3.30) for Case 1) with some slight modifications, it is easy to see that (3.30) also holds true for Case 2), which completes the proof of (3.30).

Step 3) By (3.30), the mutual disjointness of $\left\{Q_{i}^{k}\right\}_{i}$ for any fixed $k \in \mathbb{Z}$, Lemma 3.3(iv) and (3.2), we know that

$$
\begin{aligned}
\|f\|_{H_{A}^{p, q}\left(\mathbb{R}^{n}\right)} & \lesssim\left[\sum_{k \in \mathbb{Z}}\left(\sum_{i}\left|\lambda_{i}^{k}\right|^{p}\right)^{q / p}\right]^{1 / q} \sim\left[\sum_{k \in \mathbb{Z}}\left(\sum_{i} 2^{k p}\left|B_{i}^{k}\right|\right)^{q / p}\right]^{1 / q} \\
& \sim\left[\sum_{k \in \mathbb{Z}} 2^{k q}\left(\sum_{i}\left|Q_{i}^{k}\right|\right)^{q / p}\right]^{1 / q} \lesssim\left(\sum_{k \in \mathbb{Z}} 2^{k q}\left|\Omega_{k}\right|^{q / p}\right)^{1 / q} \sim\left\|S_{\psi}(f)\right\|_{L^{p, q}\left(\mathbb{R}^{n}\right)}
\end{aligned}
$$

with the usual modification made when $q=\infty$, which implies that $f \in H_{A}^{p, q}\left(\mathbb{R}^{n}\right)$ and (3.16) holds true. This finishes the proof of the sufficiency of Theorem 3.4(i).

Remark 3.3. Let all symbols be the same as those used in the proof of the sufficiency of Theorem 3.4(i). Observe that, in the original proof of the sufficiency of Theorem 3.4(i) (namely, [79, Theorem 2.7]), the authors used the following two equalities: for any $f \in$ $\mathcal{S}_{0}^{\prime}\left(\mathbb{R}^{n}\right)$ such that $S(f) \in L^{p, q}\left(\mathbb{R}^{n}\right)$, and any $x \in \mathbb{R}^{n}$,

$$
\int_{\mathbb{R}_{+}^{n+1}} f * \psi_{t}(y) * \theta_{t}(x-y) d y d m(t)=\sum_{k \in \mathbb{Z}} \sum_{i} \int_{B_{k, i}} f * \psi_{t}(y) * \theta_{t}(x-y) d y d m(t)
$$

in $\mathcal{S}^{\prime}\left(\mathbb{R}^{n}\right)$ (see [79, p. 15]) and, for each $k \in \mathbb{Z}, i$ and $x \in \mathbb{R}^{n}$,

$$
\int_{B_{k, i}} f * \psi_{t}(y) * \theta_{t}(x-y) d y d m(t)=\sum_{Q \subset Q_{i}^{k}, Q \in \mathfrak{Q}_{k}} \int_{\widehat{Q}} f * \psi_{t}(y) * \theta_{t}(x-y) d y d m(t)
$$

in $\mathcal{S}^{\prime}\left(\mathbb{R}^{n}\right)$ (see $[79,(3.22)]$ ); however, the authors therein did not prove these two equalities. In the present article, we present the proofs of both (3.39) and (3.40) and hence seal these gaps existing in the original proof of the sufficiency of [79, Theorem 2.7]; see, respectively, the proofs of (3.31) and (3.22) above for the details. 


\subsection{Real interpolation of $H_{A}^{p, q}\left(\mathbb{R}^{n}\right)$}

In this subsection, as an application of the atomic decomposition of the anisotropic Hardy-Lorentz space $H_{A}^{p, q}\left(\mathbb{R}^{n}\right)$, we give the real interpolation properties on $H_{A}^{p, q}\left(\mathbb{R}^{n}\right)$; see [77, Subsection 6.1].

We first recall some basic notions about the real interpolation (see $[6,102])$. Assume that $\left(X_{1}, X_{2}\right)$ is a compatible couple of quasi-normed spaces, namely, $X_{1}$ and $X_{2}$ are two quasi-normed linear spaces which are continuously embedded in some larger topological vector space. Let

$$
X_{1}+X_{2}:=\left\{f_{1}+f_{2}: f_{1} \in X_{1}, f_{2} \in X_{2}\right\} .
$$

For any $t \in(0, \infty]$, the Peetre $K$-functional on $X_{1}+X_{2}$ is defined by setting, for any $f \in X_{1}+X_{2}$,

$$
K\left(t, f ; X_{1}, X_{2}\right):=\inf \left\{\left\|f_{1}\right\|_{X_{1}}+t\left\|f_{2}\right\|_{X_{2}}: f=f_{1}+f_{2}, f_{1} \in X_{1} \text { and } f_{2} \in X_{2}\right\} .
$$

Moreover, for any $\theta \in(0,1)$ and $q \in(0, \infty]$, the real interpolation space $\left(X_{1}, X_{2}\right)_{\theta, q}$ is defined as

$$
\left(X_{1}, X_{2}\right)_{\theta, q}:=\left\{f \in X_{1}+X_{2}:\|f\|_{\theta, q}:=\left[\int_{0}^{\infty}\left\{t^{-\theta} K\left(t, f ; X_{1}, X_{2}\right)\right\}^{q} \frac{d t}{t}\right]^{1 / q}<\infty\right\}
$$

with the usual modification made when $q=\infty$.

The following Theorem 3.6 is just [77, Theorem 6.1].

Theorem 3.6. Let $p \in(0, \infty)$ and $q_{1}, q, q_{2} \in(0, \infty]$.

(i) If $p_{1}, p_{2} \in(0, \infty)$ satisfy $p_{1} \neq p_{2}$ and $1 / p=(1-\theta) / p_{1}+\theta / p_{2}$ with $\theta \in(0,1)$, then

$$
\left(H_{A}^{p_{1}, q_{1}}\left(\mathbb{R}^{n}\right), H_{A}^{p_{2}, q_{2}}\left(\mathbb{R}^{n}\right)\right)_{\theta, q}=H_{A}^{p, q}\left(\mathbb{R}^{n}\right) .
$$

(ii) If $1 / q=(1-\theta) / q_{1}+\theta / q_{2}$, where $\theta \in(0,1)$, then

$$
\left(H_{A}^{p, q_{1}}\left(\mathbb{R}^{n}\right), H_{A}^{p, q_{2}}\left(\mathbb{R}^{n}\right)\right)_{\theta, q}=H_{A}^{p, q}\left(\mathbb{R}^{n}\right) .
$$

As an immediate consequence of Theorem 3.6(i), we easily obtain the following conclusion; see also [77, Corollary 6.5].

Corollary 3.1. Let $q \in(0, \infty]$. Assume that $p, p_{1}, p_{2} \in(0, \infty)$ satisfy $p_{1} \neq p_{2}$ and $1 / p=$ $(1-\theta) / p_{1}+\theta / p_{2}$ with $\theta \in(0,1)$. Then

$$
\left(H_{A}^{p_{1}}\left(\mathbb{R}^{n}\right), H_{A}^{p_{2}}\left(\mathbb{R}^{n}\right)\right)_{\theta, q}=H_{A}^{p, q}\left(\mathbb{R}^{n}\right),
$$

where both $H_{A}^{p_{1}}\left(\mathbb{R}^{n}\right)$ and $H_{A}^{p_{2}}\left(\mathbb{R}^{n}\right)$ denote the anisotropic Hardy spaces of Bownik [12]. 
Remark 3.4. (i) If $A:=d \mathrm{I}_{n \times n}$ for some $d \in \mathbb{R}$ with $|d| \in(1, \infty)$, here and thereafter, $\mathrm{I}_{n \times n}$ denotes the $n \times n$ unit matrix, then $H_{A}^{p_{i}, q_{i}}\left(\mathbb{R}^{n}\right), H_{A}^{p, q_{i}}\left(\mathbb{R}^{n}\right), i \in\{1,2\}$, and $H_{A}^{p, q}\left(\mathbb{R}^{n}\right)$ in Theorem 3.6 become the classical isotropic Hardy-Lorentz spaces. In this case, by Theorem 3.6(i), we know that

$$
\left(H^{p_{1}, q_{1}}\left(\mathbb{R}^{n}\right), H^{p_{2}, q_{2}}\left(\mathbb{R}^{n}\right)\right)_{\theta, q}=H^{p, q}\left(\mathbb{R}^{n}\right)
$$

with all indexes as in Theorem 3.6(i), which is a well-known real interpolation result for classical isotropic Hardy-Lorentz spaces (see [37, p. 75, (2)]). In addition, by Theorem 3.6(ii), we have

$$
\left(H^{p, q_{1}}\left(\mathbb{R}^{n}\right), H^{p, q_{2}}\left(\mathbb{R}^{n}\right)\right)_{\theta, q}=H^{p, q}\left(\mathbb{R}^{n}\right)
$$

with all indexes as in Theorem 3.6(ii), which generalizes [1, Theorem 2.5].

(ii) If $A$ is as in (i) of this remark, then $H_{A}^{p_{1}}\left(\mathbb{R}^{n}\right), H_{A}^{p_{2}}\left(\mathbb{R}^{n}\right)$ and $H_{A}^{p, q}\left(\mathbb{R}^{n}\right)$ in Corollary 3.1 become, respectively, the classical isotropic Hardy and Hardy-Lorentz spaces. In this case, by Corollary 3.1, we have

$$
\left(H^{p_{1}}\left(\mathbb{R}^{n}\right), H^{p_{2}}\left(\mathbb{R}^{n}\right)\right)_{\theta, q}=H^{p, q}\left(\mathbb{R}^{n}\right) \quad \text { with all indexes as in Corollary 3.1. }
$$

In particular, $\left(H^{p_{1}}\left(\mathbb{R}^{n}\right), H^{p_{2}}\left(\mathbb{R}^{n}\right)\right)_{\theta, p}=H^{p}\left(\mathbb{R}^{n}\right)$, provided that $1 / p=(1-\theta) / p_{1}+$ $\theta / p_{2}, \theta \in(0,1)$.

(iii) For any given $p \in(1, \infty)$ and $q \in(0, \infty]$, from Corollary 3.1 and [84, Theorem 3], we deduce that

$$
H_{A}^{p, q}\left(\mathbb{R}^{n}\right)=\left(H_{A}^{p_{1}}\left(\mathbb{R}^{n}\right), H_{A}^{p_{2}}\left(\mathbb{R}^{n}\right)\right)_{\theta, q}=\left(L^{p_{1}}\left(\mathbb{R}^{n}\right), L^{p_{2}}\left(\mathbb{R}^{n}\right)\right)_{\theta, q}=L^{p, q}\left(\mathbb{R}^{n}\right),
$$

where $p_{1}, p_{2} \in(1, \infty)$ with $p_{1} \neq p_{2}$ and $\theta \in(0,1)$ such that $1 / p=(1-\theta) / p_{1}+$ $\theta / p_{2}$.

\subsection{Applications to the boundedness of Calderón-Zygmund operators}

As another application, in this subsection, we present the boundedness of anisotropic Calderón-Zygmund operators. To this end, we first recall the notion of anisotropic Calderón-Zygmund operators from [12] as follows.

Definition 3.8. Let $\delta \in\left(0, \frac{\ln \lambda_{-}}{\ln b}\right], \tau$ be as in (2.2) and $T$ a linear bounded operator on $L^{2}\left(\mathbb{R}^{n}\right)$. Then

(i) $T$ is called an anisotropic convolutional $\delta$-type Calderón-Zygmund operator if its kernel $k \in \mathcal{S}^{\prime}\left(\mathbb{R}^{n}\right)$ coincides with a locally integrable function on $\mathbb{R}^{n} \backslash\left\{\overrightarrow{0}_{n}\right\}$ and 
satisfies that there exists a positive constant $C$ such that, for any $x, y \in \mathbb{R}^{n}$ with $\rho(x)>b^{2 \tau} \rho(y)$,

$$
|k(x-y)-k(x)| \leq C \frac{[\rho(y)]^{\delta}}{[\rho(x)]^{1+\delta}}
$$

and, for any $f \in L^{2}\left(\mathbb{R}^{n}\right), T(f)(x):=$ p.v. $k * f(x)$.

(ii) $T$ is called an anisotropic non-convolutional $\delta$-type Calderón-Zygmund operator if it satisfies that, for any $f \in L^{2}\left(\mathbb{R}^{n}\right)$ with compact support and $x \notin \operatorname{supp} f$,

$$
T(f)(x)=\int_{\operatorname{supp} f} \mathcal{K}(x, y) f(y) d y,
$$

where $\mathcal{K}$ denotes a standard kernel on $\left(\mathbb{R}^{n} \times \mathbb{R}^{n}\right) \backslash\left\{(x, x): x \in \mathbb{R}^{n}\right\}$ in the following sense: there exists a positive constant $C$ such that, for any $x, y, \widetilde{x}, \widetilde{y} \in \mathbb{R}^{n}$,

$$
\begin{array}{ll}
|\mathcal{K}(x, y)| \leq \frac{C}{\rho(x-y)}, & \text { when } x \neq y, \\
|\mathcal{K}(x, y)-\mathcal{K}(x, \widetilde{y})| \leq C \frac{[\rho(y-\widetilde{y})]^{\delta}}{[\rho(x-y)]^{1+\delta}}, & \text { when } \rho(x-y) \geq b^{2 \tau} \rho(y-\widetilde{y}), \\
|\mathcal{K}(x, y)-\mathcal{K}(\widetilde{x}, y)| \leq C \frac{[\rho(x-\widetilde{x})]^{\delta}}{[\rho(x-y)]^{1+\delta}}, & \text { when } \rho(x-y) \geq b^{2 \tau} \rho(x-\widetilde{x}) .
\end{array}
$$

In [77, Theorem 6.8 and Remark 6.10], the authors obtained the boundedness of anisotropic $\delta$-type Calderón-Zygmund operators from $H_{A}^{p}\left(\mathbb{R}^{n}\right)$ to $L^{p, \infty}\left(\mathbb{R}^{n}\right)$ (or to $\left.H_{A}^{p, \infty}\left(\mathbb{R}^{n}\right)\right)$ in the critical case.

Theorem 3.7. Let $\delta \in\left(0, \frac{\ln \lambda_{-}}{\ln b}\right]$ and $p=\frac{1}{1+\delta}$.

(i) If $T$ is an anisotropic convolutional (or non-convolutional) $\delta$-type Calderón-Zygmund operator, then there exists a positive constant $C$ such that, for any $f \in H_{A}^{p}\left(\mathbb{R}^{n}\right)$,

$$
\|T(f)\|_{L^{p, \infty}\left(\mathbb{R}^{n}\right)} \leq C\|f\|_{H_{A}^{p}\left(\mathbb{R}^{n}\right)} .
$$

(ii) If $T$ is either an anisotropic convolutional $\delta$-type Calderón-Zygmund operator or nonconvolutional $\delta$-type Calderón-Zygmund operator satisfying $T^{*} 1=0$ (namely, for any $\widetilde{a} \in L^{1}\left(\mathbb{R}^{n}\right)$ with compact support, if $\int_{\mathbb{R}^{n}} \widetilde{a}(x) d x=0$, then $\left.\int_{\mathbb{R}^{n}} T(\widetilde{a})(x) d x=0\right)$, then there exists a positive constant $\widetilde{C}$ such that, for any $f \in H_{A}^{p}\left(\mathbb{R}^{n}\right)$,

$$
\|T(f)\|_{H_{A}^{p, \infty}\left(\mathbb{R}^{n}\right)} \leq \widetilde{C}\|f\|_{H_{A}^{p}\left(\mathbb{R}^{n}\right)} .
$$


Remark 3.5. (i) If $A$ is as in Remark 3.4(i), then $\frac{\ln \lambda_{-}}{\ln b}=\frac{1}{n}$, and $H_{A}^{p}\left(\mathbb{R}^{n}\right)$ and $H_{A}^{p, \infty}\left(\mathbb{R}^{n}\right)$ become, respectively, the classical isotropic Hardy and weak Hardy spaces. In this case, by Theorem 3.7(ii), we know that, if $\delta \in(0,1], p=\frac{n}{n+\delta}$ and $T$ is the CalderónZygmund operator satisfying all conditions of Theorem 3.7(ii) with (3.42) replaced by

$$
|k(x-y)-k(x)| \lesssim \frac{|y|^{\delta}}{|x|^{n+\delta}}, \quad|x| \geq 2|y|,
$$

where the implicit positive constant is independent of $x$ and $y$, then $T$ is bounded from $H^{\frac{n}{n+\delta}}\left(\mathbb{R}^{n}\right)$ to $H^{\frac{n}{n+\delta}, \infty}\left(\mathbb{R}^{n}\right)$, which is just [72, Theorem 1]. Here $\frac{n}{n+\delta}$ is called the critical index. In this sense, Theorem 3.7(i) also establishes the boundedness of Calderón-Zygmund operators from $H_{A}^{p}\left(\mathbb{R}^{n}\right)$ to $L^{p, \infty}\left(\mathbb{R}^{n}\right)$ in the critical case under the present anisotropic setting.

(ii) Let $\delta \in\left(0, \frac{\ln \lambda_{-}}{\ln b}\right]$ and $p \in\left(\frac{1}{1+\delta}, 1\right]$. For the boundedness of anisotropic $\delta$-type Calderón-Zygmund operators from $H_{A}^{p}\left(\mathbb{R}^{n}\right)$ to itself (or to $L^{p}\left(\mathbb{R}^{n}\right)$ ), we refer the reader to [12, p. 68, Theorem 9.8 and p. 69, Theorem 9.9].

The following boundedness of $\delta$-type Calderón-Zygmund operators from $H_{A}^{p, q}\left(\mathbb{R}^{n}\right)$ to itself (or to $L^{p, q}\left(\mathbb{R}^{n}\right)$ ) was presented in [77, Theorem 6.16].

Theorem 3.8. Let $\delta \in\left(0, \frac{\ln \lambda_{-}}{\ln b}\right], p \in\left(\frac{1}{1+\delta}, 1\right]$ and $q \in(0, \infty]$.

(i) If $T$ is as in Theorem 3.7(i), then there exists a positive constant $C$ such that, for any $f \in H_{A}^{p, q}\left(\mathbb{R}^{n}\right)$,

$$
\|T(f)\|_{L^{p, q}\left(\mathbb{R}^{n}\right)} \leq C\|f\|_{H_{A}^{p, q}\left(\mathbb{R}^{n}\right)} \cdot
$$

(ii) If $T$ is as in Theorem 3.7(ii), then there exists a positive constant $\widetilde{C}$ such that, for any $f \in H_{A}^{p, q}\left(\mathbb{R}^{n}\right)$,

$$
\|T(f)\|_{H_{A}^{p, q}\left(\mathbb{R}^{n}\right)} \leq \widetilde{C}\|f\|_{H_{A}^{p, q}\left(\mathbb{R}^{n}\right)} .
$$

Remark 3.6. If $A$ is as in Remark 3.4(i), then $\frac{\ln \lambda_{-}}{\ln b}=\frac{1}{n}$ and $T$ becomes the classical $\delta$-type Calderón-Zygmund operator. In this case, we know that, if $\delta \in(0,1], p \in\left(\frac{n}{n+\delta}, 1\right]$ and $q \in(0, \infty]$, then Theorem 3.8 implies that $T$ is bounded from the classical Hardy-Lorentz space $H^{p, q}\left(\mathbb{R}^{n}\right)$ to itself (or to $L^{p, q}\left(\mathbb{R}^{n}\right)$ ). Moreover, when $p=q$, (i) and (ii) of Theorem 3.8 imply the boundedness of classical $\delta$-type Calderón-Zygmund operators from $H^{p}\left(\mathbb{R}^{n}\right)$ to $L^{p}\left(\mathbb{R}^{n}\right)$, respectively, from $H^{p}\left(\mathbb{R}^{n}\right)$ to $H^{p}\left(\mathbb{R}^{n}\right)$ for $\delta \in(0,1]$ and $p \in\left(\frac{n}{n+\delta}, 1\right]$, which is a well-known result (see, for instance, $[3,96])$.

Theorem 3.9. Let $p \in(0,1], q \in(p, \infty], r \in(1, \infty)$ and $k$ be the kernel of some CalderónZygmund operator T. Moreover, assume that $T$ is bounded from $L^{r}\left(\mathbb{R}^{n}\right)$ to $L^{r, \infty}\left(\mathbb{R}^{n}\right)$ and $\omega_{p}$ satisfies a Dini-type condition of order $q /(q-p)$, namely,

$$
A_{(p, q)}:=\left\{\int_{0}^{1}\left[\omega_{p}(\delta)\right]^{q /(q-p)} \frac{d \delta}{\delta}\right\}^{(q-p) / q}<\infty
$$


where, for any $\delta \in(0,1]$,

$$
\begin{gathered}
\omega_{p}(\delta):=\sup _{B} \frac{1}{|B|} \int_{\rho\left(x-y_{B}\right)>\frac{b^{2} \tau}{\delta}|B|}\left[\int_{B}\left|k(x, y)-\sum_{|\beta| \leq N}\left(y-y_{B}\right)^{\beta} k_{\beta}\left(x, y_{B}\right)\right| d y\right]^{p} d x, \\
N:=\left\lfloor(1 / p-1) \frac{\ln b}{\ln \lambda_{-}}\right\rfloor, \beta:=\left(\beta_{1}, \cdots, \beta_{n}\right) \in \mathbb{Z}_{+}^{n}, \\
k_{\beta}\left(x, y_{B}\right):=\left.\frac{1}{\beta !} \partial^{\beta} k(x, y)\right|_{y=y_{B}}
\end{gathered}
$$

and the supremum is taken over all dilated balls $B \in \mathfrak{B}$ centered at $y_{B}$. Then there exists a positive constant $C$ such that, for any $f \in H_{A}^{p, q}\left(\mathbb{R}^{n}\right)$,

$$
\|T(f)\|_{L^{p, \infty}\left(\mathbb{R}^{n}\right)} \leq C\left[A_{(p, q)}\right]^{1 / p}\|f\|_{H_{A}^{p, q}\left(\mathbb{R}^{n}\right)} .
$$

Remark 3.7. (i) If $A$ is as in Remark 3.4(i), then $\frac{\ln b}{\ln \lambda_{-}}=n, N=\lfloor n(1 / p-1)\rfloor$ and $H_{A}^{p, q}\left(\mathbb{R}^{n}\right)$ becomes the classical Hardy-Lorentz space. In this case, Theorem 3.9 is just [1, Theorem 2.2].

(ii) It is well known that the Hörmander condition implies the boundedness of the Calderón-Zygmund operator $T$ from $H_{A}^{1}\left(\mathbb{R}^{n}\right)$ to $L^{1}\left(\mathbb{R}^{n}\right)$. Observe that $H_{A}^{1}\left(\mathbb{R}^{n}\right) \varsubsetneqq$ $H_{A}^{1, q}\left(\mathbb{R}^{n}\right)$ with $q \in(1, \infty]$. Thus, to define $T$ on $H_{A}^{1, q}\left(\mathbb{R}^{n}\right)$ with $q \in(1, \infty]$, it is natural to require $T$ to satisfy some conditions stronger than the usual Hörmander condition. This was accomplished by the variable dilations (the Dini-type condition (3.43)) of Fefferman and Soria [39] (see also [1]). Moreover, recall that we consider $p=\frac{1}{1+\delta}$ or $p \in\left(\frac{1}{1+\delta}, 1\right]$ with $\delta \in\left(0, \frac{\ln \lambda_{-}}{\ln b}\right]$ in Theorem 3.7 and Remark 3.5(ii), which implies

$$
N=\left\lfloor\frac{\ln b}{\ln \lambda_{-}}\left(\frac{1}{p}-1\right)\right\rfloor \leq 1
$$

While, in Theorem 3.9, we consider $p \in(0,1]$. If $p$ becomes smaller, then $N$ becomes larger. Thus, more regularity of the kernel of $T$ is needed. This justifies the definition of $\omega_{p}(\delta)$ in Theorem 3.9.

(iii) We point out that both the dual spaces and the wavelet characterizations of anisotropic Hardy-Lorentz spaces $H_{A}^{p, q}\left(\mathbb{R}^{n}\right)$ are still unknown. In addition, one can also consider the corresponding multiplier theorem on the space $H_{A}^{p, q}\left(\mathbb{R}^{n}\right)$, similar to that studied by Wang in [110] on anisotropic Hardy spaces $H_{A}^{p}\left(\mathbb{R}^{n}\right)$.

\section{Anisotropic variable Hardy spaces}

In this section, we first recall the definition of anisotropic variable Hardy spaces $H_{A}^{p(\cdot)}\left(\mathbb{R}^{n}\right)$ and then give the real-variable theory of these spaces; see [75]. 


\subsection{Definition of anisotropic variable Hardy spaces}

For any measurable function $p(\cdot): \mathbb{R}^{n} \rightarrow(0, \infty]$, let

$$
p_{-}:=\underset{x \in \mathbb{R}^{n}}{\operatorname{essinf}} p(x), \quad p_{+}:=\underset{x \in \mathbb{R}^{n}}{\operatorname{ess} \sup } p(x) \quad \text { and } \quad \underline{p}:=\min \left\{p_{-}, 1\right\} .
$$

Denote by $\mathcal{P}\left(\mathbb{R}^{n}\right)$ the set of all measurable functions $p(\cdot)$ satisfying $0<p_{-} \leq p_{+}<\infty$.

For any $p(\cdot) \in \mathcal{P}\left(\mathbb{R}^{n}\right)$, the variable Lebesgue space $L^{p(\cdot)}\left(\mathbb{R}^{n}\right)$ is defined to be the set of all measurable functions $f$ such that $\varrho_{p(\cdot)}(f)<\infty$, equipped with the quasi-norm $\|f\|_{L^{p(\cdot)}\left(\mathbb{R}^{n}\right)}$, where, for any measurable function $f$, the modular functional $\varrho_{p(\cdot)}(f)$ and the Luxembourg (also called Luxembourg-Nakano) quasi-norm $\|f\|_{L^{p(\cdot)}\left(\mathbb{R}^{n}\right)}$ of $f$ are defined, respectively, by setting

$$
\begin{aligned}
& \varrho_{p(\cdot)}(f):=\int_{\mathbb{R}^{n}}|f(x)|^{p(x)} d x, \\
& \|f\|_{L^{p(\cdot)}\left(\mathbb{R}^{n}\right)}:=\inf \left\{\lambda \in(0, \infty): \varrho_{p(\cdot)}(f / \lambda) \leq 1\right\},
\end{aligned}
$$

see, for instance, $[22,26]$.

Let $C^{\log }\left(\mathbb{R}^{n}\right)$ be the set of all functions $p(\cdot) \in \mathcal{P}\left(\mathbb{R}^{n}\right)$ satisfying the globally log-Hölder continuity condition, namely, there exist $C_{\log }(p), C_{\infty} \in(0, \infty)$ and $p_{\infty} \in \mathbb{R}$ such that, for any $x, y \in \mathbb{R}^{n}$,

$$
|p(x)-p(y)| \leq \frac{C_{\log }(p)}{\log (e+1 / \rho(x-y))}
$$

and

$$
\left|p(x)-p_{\infty}\right| \leq \frac{C_{\infty}}{\log (e+\rho(x))} .
$$

The following anisotropic variable Hardy space was originally introduced in [75, Definition 2.4]

Definition 4.1. Let $p(\cdot) \in C^{\log }\left(\mathbb{R}^{n}\right)$ and

$$
N \in \mathbb{N} \cap\left[\left\lfloor\left(\frac{1}{\underline{p}}-1\right) \frac{\ln b}{\ln \lambda_{-}}\right\rfloor+2, \infty\right),
$$

where $\underline{p}$ is as in (4.1). The anisotropic variable Hardy space $H_{A}^{p(\cdot)}\left(\mathbb{R}^{n}\right)$ is defined by setting

$$
H_{A}^{p(\cdot)}\left(\mathbb{R}^{n}\right):=\left\{f \in \mathcal{S}^{\prime}\left(\mathbb{R}^{n}\right): M_{N}(f) \in L^{p(\cdot)}\left(\mathbb{R}^{n}\right)\right\}
$$

and, for any $f \in H_{A}^{p(\cdot)}\left(\mathbb{R}^{n}\right)$, let $\|f\|_{H_{A}^{p(\cdot)}\left(\mathbb{R}^{n}\right)}:=\left\|M_{N}(f)\right\|_{L^{p(\cdot)}\left(\mathbb{R}^{n}\right)}$, where $M_{N}(f)$ denotes the non-tangential grand maximal function of $f$ as in (3.4). 
Remark 4.1. (i) The quasi-norm of $H_{A}^{p(\cdot)}\left(\mathbb{R}^{n}\right)$ in Definition 4.1 depends on $N$, however, by Theorem 4.1 below, we know that the space $H_{A}^{p(\cdot)}\left(\mathbb{R}^{n}\right)$ is independent of the choice of $N$ as long as $N$ is as in (4.4). In addition, when $p(\cdot) \equiv p \in(0, \infty)$, the space $H_{A}^{p(\cdot)}\left(\mathbb{R}^{n}\right)$ becomes the anisotropic Hardy space $H_{A}^{p}\left(\mathbb{R}^{n}\right)$ from [12] and, when $A:=d \mathrm{I}_{n \times n}$ for some $d \in \mathbb{R}$ with $|d| \in(1, \infty)$, the space $H_{A}^{p(\cdot)}\left(\mathbb{R}^{n}\right)$ goes back to the classical variable Hardy space (see $[23,87])$.

(ii) Recall that, in [51], Huang et al. established various real-variable characterizations of anisotropic mixed-norm Hardy spaces $H_{A}^{\vec{p}}\left(\mathbb{R}^{n}\right)$. We point out that the integrable exponent of the Hardy space $H_{A}^{\vec{p}}\left(\mathbb{R}^{n}\right)$ from [51] is a vector $\vec{p} \in(0, \infty)^{n}$, whose associated basic function space is the mixed-norm Lebesgue space $L^{\vec{p}}\left(\mathbb{R}^{n}\right)$, which has different orders of integrability in different variables; however, the integrable exponent of the anisotropic variable Hardy space $H_{A}^{p(\cdot)}\left(\mathbb{R}^{n}\right)$ is a variable exponent function,

$$
p(\cdot): \mathbb{R}^{n} \rightarrow(0, \infty]
$$

satisfying the so-called globally log-Hölder continuity condition, whose associated basic function space is the variable Lebesgue space $L^{p(\cdot)}\left(\mathbb{R}^{n}\right)$. Obviously, $L^{\vec{p}}\left(\mathbb{R}^{n}\right)$ and $L^{p(\cdot)}\left(\mathbb{R}^{n}\right)$ cannot cover each other, so do the anisotropic mixed-norm Hardy space $H_{A}^{\vec{p}}\left(\mathbb{R}^{n}\right)$ of [51] and the Hardy space $H_{A}^{p(\cdot)}\left(\mathbb{R}^{n}\right)$ in Definition 4.1.

(iii) Let $H^{p(\cdot), q(\cdot)}\left(\mathbb{R}^{n}, A\right)$ be the anisotropic variable Hardy-Lorentz space defined via the variable Lorentz space $\mathcal{L}^{p(\cdot), q(\cdot)}\left(\mathbb{R}^{n}\right)$ (see $[2,31]$ ), where

$$
p(\cdot), q(\cdot):(0, \infty) \rightarrow(0, \infty)
$$

are two measurable functions (see also Remark 3.1(iii)). As was mentioned in [59, Remark 2.6], the space $\mathcal{L}^{p(\cdot), q(\cdot)}\left(\mathbb{R}^{n}\right)$ in [31] never goes back to the space $L^{p(\cdot)}\left(\mathbb{R}^{n}\right)$, because the variable exponent $p(\cdot)$ in $\mathcal{L}^{p(\cdot), q(\cdot)}\left(\mathbb{R}^{n}\right)$ is only defined on $(0, \infty)$ while not on $\mathbb{R}^{n}$. Thus, it is easy to see that the space $H_{A}^{p(\cdot)}\left(\mathbb{R}^{n}\right)$, in this article, is not covered by the space $H^{p(\cdot), q(\cdot)}\left(\mathbb{R}^{n}, A\right)$ in [2].

\subsection{Equivalent characterizations of $H_{A}^{p(\cdot)}\left(\mathbb{R}^{n}\right)$}

In [75, Theorem 3.10], the authors established the radial or the non-tangential maximal function characterizations of $H_{A}^{p(\cdot)}\left(\mathbb{R}^{n}\right)$ as follows.

Theorem 4.1. Let $p(\cdot) \in C^{\log }\left(\mathbb{R}^{n}\right)$ and $\phi \in \mathcal{S}\left(\mathbb{R}^{n}\right)$ satisfying $\int_{\mathbb{R}^{n}} \phi(x) d x \neq 0$. Then, for any $f \in \mathcal{S}^{\prime}\left(\mathbb{R}^{n}\right)$, the following statements are mutually equivalent:

(i) $f \in H_{A}^{p(\cdot)}\left(\mathbb{R}^{n}\right)$; 
(ii) $M_{\phi}(f) \in L^{p(\cdot)}\left(\mathbb{R}^{n}\right)$;

(iii) $M_{\phi}^{0}(f) \in L^{p(\cdot)}\left(\mathbb{R}^{n}\right)$,

where $M_{\phi}(f)$ and $M_{\phi}^{0}(f)$ are, respectively, as in (3.3) and (3.6). Moreover, there exist two positive constants $C$ and $\widetilde{C}$, independent of $f$, such that

$$
\|f\|_{H_{A}^{p(\cdot)}\left(\mathbb{R}^{n}\right)} \leq C\left\|M_{\phi}^{0}(f)\right\|_{L^{p(\cdot)}\left(\mathbb{R}^{n}\right)} \leq C\left\|M_{\phi}(f)\right\|_{L^{p(\cdot)}\left(\mathbb{R}^{n}\right)} \leq \widetilde{C}\|f\|_{H_{A}^{p(\cdot)}\left(\mathbb{R}^{n}\right)^{n}} .
$$

We now recall the notions of anisotropic variable atomic Hardy spaces and finite atomic Hardy spaces from [75].

Definition 4.2. Let $p(\cdot) \in C^{\log }\left(\mathbb{R}^{n}\right), q \in(1, \infty]$ and

$$
s \in\left[\left\lfloor\left(\frac{1}{p_{-}}-1\right) \frac{\ln b}{\ln \lambda_{-}}\right\rfloor, \infty\right) \cap \mathbb{Z}_{+} .
$$

(i) An anisotropic $(p(\cdot), q, s)$-atom (shortly, a $(p(\cdot), q, s)$-atom) is a measurable function $a$ on $\mathbb{R}^{n}$ satisfying

(i) $)_{1} \operatorname{supp} a:=\left\{x \in \mathbb{R}^{n}: a(x) \neq 0\right\} \subset B$, where $B \in \mathfrak{B}$ and $\mathfrak{B}$ is as in (2.1);

(i) $2\|a\|_{L^{q}\left(\mathbb{R}^{n}\right)} \leq \frac{|B|^{1 / q}}{\left\|\mathbf{1}_{B}\right\|_{L^{p(\cdot)}\left(\mathbb{R}^{n}\right)}} ;$

(i) $)_{3}$ for any $\gamma \in \mathbb{Z}_{+}^{n}$ with $|\gamma| \leq s, \int_{\mathbb{R}^{n}} a(x) x^{\gamma} d x=0$.

(ii) The anisotropic variable atomic Hardy space $H_{A}^{p(\cdot), q, s}\left(\mathbb{R}^{n}\right)$ is defined to be the set of all $f \in \mathcal{S}^{\prime}\left(\mathbb{R}^{n}\right)$ satisfying that there exist $\left\{\lambda_{i}\right\}_{i \in \mathbb{N}} \subset \mathbb{C}$ and a sequence of $(p(\cdot), q, s)$ atoms, $\left\{a_{i}\right\}_{i \in \mathbb{N}}$, supported, respectively, in $\left\{B^{(i)}\right\}_{i \in \mathbb{N}} \subset \mathfrak{B}$ such that

$$
f=\sum_{i \in \mathbb{N}} \lambda_{i} a_{i} \text { in } \mathcal{S}^{\prime}\left(\mathbb{R}^{n}\right)
$$

Moreover, for any $f \in H_{A}^{p(\cdot), q, s}\left(\mathbb{R}^{n}\right)$, let

$$
\|f\|_{H_{A}^{p(\cdot), q, s}\left(\mathbb{R}^{n}\right)}:=\inf \left\|\left\{\sum_{i \in \mathbb{N}}\left[\frac{\left|\lambda_{i}\right| \mathbf{1}_{B^{(i)}}}{\left\|\mathbf{1}_{B^{(i)}}\right\|_{L^{p(\cdot)}\left(\mathbb{R}^{n}\right)}}\right]^{\underline{p}}\right\}^{1 / \underline{p}}\right\|_{L^{p(\cdot)}\left(\mathbb{R}^{n}\right)},
$$

where the infimum is taken over all the decompositions of $f$ as above.

(iii) The anisotropic variable finite atomic Hardy space $H_{A, f i n}^{p(\cdot), q, s}\left(\mathbb{R}^{n}\right)$ is defined to be the set of all $f \in \mathcal{S}^{\prime}\left(\mathbb{R}^{n}\right)$ satisfying that there exist $I \in \mathbb{N},\left\{\lambda_{i}\right\}_{i \in[1, I] \cap \mathbb{N}} \subset \mathbb{C}$ 
and a finite sequence of $(p(\cdot), q, s)$-atoms, $\left\{a_{i}\right\}_{i \in[1, I] \cap \mathbb{N}}$, supported, respectively, in $\left\{B^{(i)}\right\}_{i \in[1, I] \cap \mathbb{N}} \subset \mathfrak{B}$ such that

$$
f=\sum_{i=1}^{I} \lambda_{i} a_{i} \quad \text { in } \mathcal{S}^{\prime}\left(\mathbb{R}^{n}\right) .
$$

Moreover, for any $f \in H_{A, \text { fin }}^{p(\cdot), s}\left(\mathbb{R}^{n}\right)$, let

$$
\|f\|_{H_{A, \text { fin }}^{p(\cdot),\left(\mathbb{R}^{n}\right)}}:=\inf \left\|\left\{\sum_{i=1}^{I}\left[\frac{\left|\lambda_{i}\right| \mathbf{1}_{B^{(i)}}}{\left\|\mathbf{1}_{B^{(i)}}\right\|_{L^{p(\cdot)}\left(\mathbb{R}^{n}\right)}}\right]^{\underline{p}}\right\}^{1 / \underline{p}}\right\|_{L^{p(\cdot)}\left(\mathbb{R}^{n}\right)},
$$

where the infimum is taken over all the decompositions of $f$ as above.

The following atomic and finite atomic characterizations of $H_{A}^{p(\cdot)}\left(\mathbb{R}^{n}\right)$ were just [75, Theorems 4.8 and 5.4].

Theorem 4.2. Let $p(\cdot) \in C^{\log }\left(\mathbb{R}^{n}\right), q \in\left(\max \left\{p_{+}, 1\right\}, \infty\right]$ with $p_{+}$as in $(4.1)$, s be as in (4.5) and $N$ as in (4.4). Then

(i) $H_{A}^{p(\cdot)}\left(\mathbb{R}^{n}\right)=H_{A}^{p(\cdot), q, s}\left(\mathbb{R}^{n}\right)$ with equivalent quasi-norms;

(ii) $\|\cdot\|_{H_{A, \text { fin }}^{p(\cdot), s}\left(\mathbb{R}^{n}\right)}$ and $\|\cdot\|_{H_{A}^{p(\cdot)}\left(\mathbb{R}^{n}\right)}$ are equivalent quasi-norms on $H_{A, \text { fin }}^{p(\cdot), q, s}\left(\mathbb{R}^{n}\right)$ for each $q \in$ $\left(\max \left\{p_{+}, 1\right\}, \infty\right)$;

(iii) $\|\cdot\|_{H_{A, \text { fin }}^{p(\cdot), s}\left(\mathbb{R}^{n}\right)}$ and $\|\cdot\|_{H_{A}^{p(\cdot)}\left(\mathbb{R}^{n}\right)}$ are equivalent quasi-norms on $H_{A, \mathrm{fin}}^{p(\cdot), \infty, s}\left(\mathbb{R}^{n}\right) \cap C\left(\mathbb{R}^{n}\right)$.

\subsection{Littlewood-Paley function characterizations of $H_{A}^{p(\cdot)}\left(\mathbb{R}^{n}\right)$}

In this subsection, we present the characterizations of $H_{A}^{p(\cdot)}\left(\mathbb{R}^{n}\right)$ in terms of the anisotropic Lusin area function, the anisotropic Littlewood-Paley $g$-function or $g_{\lambda}^{*}$ function; see [75]. Meanwhile, we also correct some errors existing in the proof of the sufficiency of [75, Theorem 6.1], namely, the Lusin area function characterizations of $H_{A}^{p(\cdot)}\left(\mathbb{R}^{n}\right)$.

First, recall that the following Fefferman-Stein vector-valued inequality of the maximal operator $M_{\mathrm{HL}}$ on the variable Lebesgue space $L^{p(\cdot)}\left(\mathbb{R}^{n}\right)$ was established in [78, Lemma 4.3].

Lemma 4.1. Let $r \in(1, \infty]$. Assume that $p(\cdot) \in C^{\log }\left(\mathbb{R}^{n}\right)$ satisfies $1<p_{-} \leq p_{+}<\infty$. Then there exists a positive constant $C$ such that, for any sequence $\left\{f_{k}\right\}_{k \in \mathbb{N}}$ of measurable functions,

$$
\left\|\left\{\sum_{k \in \mathbb{N}}\left[M_{\mathrm{HL}}\left(f_{k}\right)\right]^{r}\right\}^{1 / r}\right\|_{L^{p(\cdot)}\left(\mathbb{R}^{n}\right)} \leq C\left\|\left(\sum_{k \in \mathbb{N}}\left|f_{k}\right|^{r}\right)^{1 / r}\right\|_{L^{p(\cdot)}\left(\mathbb{R}^{n}\right)}
$$


with the usual modification made when $r=\infty$, where $M_{\mathrm{HL}}$ denotes the Hardy-Littlewood maximal operator as in (3.9).

Via Lemmas 4.1 and 3.4, following the proof of Theorem 3.5 with some slight modifications, we easily obtain the following conclusion; the details are omitted.

Theorem 4.3. Let $p(\cdot) \in C^{\log }\left(\mathbb{R}^{n}\right)$ and $\theta, \psi$ be as in Lemma 3.1 with $s$ as in (4.5). Then there exists a positive constant $C$ such that, for any $f \in \mathcal{S}_{0}^{\prime}\left(\mathbb{R}^{n}\right)$,

$$
\frac{1}{C}\left\|S_{\theta}(f)\right\|_{L^{p(\cdot)}\left(\mathbb{R}^{n}\right)} \leq\left\|S_{\psi}(f)\right\|_{L^{p(\cdot)}\left(\mathbb{R}^{n}\right)} \leq C\left\|S_{\theta}(f)\right\|_{L^{p(\cdot)}\left(\mathbb{R}^{n}\right)},
$$

where $S_{\theta}(f)$ and $S_{\psi}(f)$ are the anisotropic Lusin area functions as in (3.8) defined, respectively, via $\theta$ and $\psi$.

In [75, Theorems 6.1 through 6.3], the authors characterized the space $H_{A}^{p(\cdot)}\left(\mathbb{R}^{n}\right)$, respectively, in terms of the anisotropic Lusin area function, the anisotropic LittlewoodPaley $g$-function or $g_{\lambda}^{*}$-function as follows.

Theorem 4.4. Let $p(\cdot) \in C^{\log }\left(\mathbb{R}^{n}\right)$. Then

(i) $f \in H_{A}^{p(\cdot)}\left(\mathbb{R}^{n}\right)$ if and only if $f \in \mathcal{S}_{0}^{\prime}\left(\mathbb{R}^{n}\right)$ and $S(f) \in L^{p(\cdot)}\left(\mathbb{R}^{n}\right)$. Moreover, there exists a positive constant $C$ such that, for any $f \in H_{A}^{p(\cdot)}\left(\mathbb{R}^{n}\right)$,

$$
\frac{1}{C}\|S(f)\|_{L^{p(\cdot)}\left(\mathbb{R}^{n}\right)} \leq\|f\|_{H_{A}^{p(\cdot)}\left(\mathbb{R}^{n}\right)} \leq C\|S(f)\|_{L^{p(\cdot)}\left(\mathbb{R}^{n}\right)} .
$$

(ii) The conclusion as in (i) remains true if $S(f)$ is replaced, respectively, by $g(f)$ or $g_{\lambda}^{*}(f)$ with $\lambda \in\left(1+\frac{2}{\min \left\{p_{-,}\right\}}, \infty\right)$.

To show the sufficiency of Theorem 4.4(i), we need the following lemma, whose proof is similar to that of [52, Lemma 4.7] (see also the proof of [91, Lemma 4.1]); we omit the details here.

Lemma 4.2. Let $p(\cdot) \in C^{\log }\left(\mathbb{R}^{n}\right), k_{0} \in \mathbb{Z}, \varepsilon \in(0, \infty)$ and $r \in[1, \infty] \cap\left(p_{+}, \infty\right]$ with $p_{+}$as in (4.1). Assume that $\left\{\lambda_{i}\right\}_{i \in \mathbb{N}} \subset \mathbb{C},\left\{B^{(i)}\right\}_{i \in \mathbb{N}} \subset \mathfrak{B}$ and $\left\{m_{i}^{(\varepsilon)}\right\}_{i \in \mathbb{N}} \subset L^{r}\left(\mathbb{R}^{n}\right)$ satisfy that, for any $\varepsilon \in(0, \infty)$ and $i \in \mathbb{N}$,

$$
\begin{aligned}
& \operatorname{supp} m_{i}^{(\varepsilon)}:=\left\{x \in \mathbb{R}^{n}: m_{i}^{(\varepsilon)}(x) \neq 0\right\} \subset A^{k_{0}} B^{(i)}, \\
& \left\|m_{i}^{(\varepsilon)}\right\|_{L^{r}\left(\mathbb{R}^{n}\right)} \leq \frac{\left|B^{(i)}\right|^{1 / r}}{\left\|\mathbf{1}_{B^{(i)}}\right\|_{L^{p(\cdot)}\left(\mathbb{R}^{n}\right)}}, \\
& \left\|\left\{\sum_{i \in \mathbb{N}}\left[\frac{\left|\lambda_{i}\right| \mathbf{1}_{B^{(i)}}}{\left\|\mathbf{1}_{B^{(i)}}\right\|_{L^{p(\cdot)}\left(\mathbb{R}^{n}\right)}}\right]^{\underline{p}}\right\}^{1 / \underline{p}}\right\|_{L^{p(\cdot)}\left(\mathbb{R}^{n}\right)}<\infty,
\end{aligned}
$$


with $\underline{p}$ as in (4.1). Then

$$
\left\|\liminf _{\varepsilon \rightarrow 0^{+}}\left[\sum_{i \in \mathbb{N}}\left|\lambda_{i} m_{i}^{(\varepsilon)}\right|^{\underline{p}}\right]^{1 / \underline{p}}\right\|_{L^{p(\cdot)}\left(\mathbb{R}^{n}\right)} \leq C\left\|\left\{\sum_{i \in \mathbb{N}}\left[\frac{\left|\lambda_{i}\right| \mathbf{1}_{B^{(i)}}}{\left\|\mathbf{1}_{B^{(i)}}\right\|_{L^{p(\cdot)}\left(\mathbb{R}^{n}\right)}}\right]^{\underline{p}}\right\}^{1 / \underline{p}}\right\|_{L^{p(\cdot)}\left(\mathbb{R}^{n}\right)},
$$

where $C$ is a positive constant independent of $\lambda_{i}, B^{(i)}, m_{i}^{(\varepsilon)}$ and $\varepsilon$.

Now we prove the sufficiency of Theorem 4.4(i).

Proof of the sufficiency of Theorem 4.4(i). Let $\psi$ be as in Lemma 3.1, $f \in \mathcal{S}_{0}^{\prime}\left(\mathbb{R}^{n}\right)$ and $S(f) \in$ $L^{p(\cdot)}\left(\mathbb{R}^{n}\right)$. Then, by Theorem 4.3 , we know that $S_{\psi}(f) \in L^{p(\cdot)}\left(\mathbb{R}^{n}\right)$. Thus, to show the sufficiency of Theorem 4.4(i), we need to prove that $f \in H_{A}^{p(\cdot)}\left(\mathbb{R}^{n}\right)$ and

$$
\|f\|_{H_{A}^{p(\cdot)}\left(\mathbb{R}^{n}\right)} \lesssim\left\|S_{\psi}(f)\right\|_{L^{p(\cdot)}\left(\mathbb{R}^{n}\right)} .
$$

For this purpose, for any $k \in \mathbb{Z}$, let $\Omega_{k}:=\left\{x \in \mathbb{R}^{n}: S_{\psi}(f)(x)>2^{k}\right\}$ and

$$
\mathfrak{Q}_{k}:=\left\{Q \in \mathfrak{Q}:\left|Q \cap \Omega_{k}\right|>\frac{|Q|}{2} \text { and }\left|Q \cap \Omega_{k+1}\right| \leq \frac{|Q|}{2}\right\} .
$$

Clearly, for any $Q \in \mathfrak{Q}$, there exists a unique $k \in \mathbb{Z}$ such that $Q \in \mathfrak{Q}_{k}$. Let $\left\{Q_{i}^{k}\right\}_{i}$ be the set of all maximal dyadic cubes in $\mathfrak{Q}_{k}$, namely, there exists no $Q \in \mathfrak{Q}_{k}$ such that $Q_{i}^{k} \varsubsetneqq Q$ for any $i$.

For any $Q \in \mathfrak{Q}$, let

$$
\widehat{Q}:=\left\{(y, t) \in \mathbb{R}_{+}^{n+1}:=\mathbb{R}^{n} \times(0, \infty): y \in Q, t \sim b^{v \ell(Q)+u}\right\},
$$

where $t \sim b^{v \ell(Q)+u}$ means the same as in (3.18). Obviously, $\{\widehat{Q}\}_{Q \in \mathfrak{Q}}$ are mutually disjoint and

$$
\mathbb{R}_{+}^{n+1}=\bigcup_{k \in \mathbb{Z}} \bigcup_{i} B_{k, i}
$$

where, for any $k \in \mathbb{Z}$ and $i, B_{k, i}:=\bigcup_{Q \subset Q_{i}^{k}, Q \in \mathfrak{Q}_{k}} \widehat{Q}$. Then, by Lemma 3.3(ii), we easily find that $\left\{B_{k, i}\right\}_{k \in \mathbb{Z}, i}$ are also mutually disjoint.

Let $\psi$ and $\theta$ be as in Lemma 3.1 with $s$ as in (4.5). Then $\theta$ has the vanishing moments up to order $s$ and, for any $f \in \mathcal{S}_{0}^{\prime}\left(\mathbb{R}^{n}\right)$ such that $S_{\psi}(f) \in L^{p(\cdot)}\left(\mathbb{R}^{n}\right)$, and for any $x \in \mathbb{R}^{n}$, similarly to (3.20), we have

$$
f(x)=\sum_{k \in \mathbb{Z}} f * \psi_{k} * \theta_{k}(x)=\int_{\mathbb{R}_{+}^{n+1}} f * \psi_{t}(y) * \theta_{t}(x-y) d y d m(t)
$$


in $\mathcal{S}^{\prime}\left(\mathbb{R}^{n}\right)$, where $m(t)$ denotes the counting measure on $\mathbb{R}$ as in (3.20). For each $k \in \mathbb{Z}, i$ and $x \in \mathbb{R}^{n}$, let

$$
h_{i}^{k}(x):=\int_{B_{k, i}} f * \psi_{t}(y) * \theta_{t}(x-y) d y d m(t) .
$$

Next we prove the sufficiency of Theorem 4.4(i) in three steps.

Step 1) The target of this step is to show that

$$
\sum_{k \in \mathbb{Z}} \sum_{i} h_{i}^{k} \quad \text { converges in } \mathcal{S}^{\prime}\left(\mathbb{R}^{n}\right)
$$

To this end, following the proofs of assertions (i) and (ii) in the proof of the sufficiency of Theorem 3.4(i) with some slight modifications, we conclude that, for any given $r \in$ $\left(\max \left\{p_{+}, 1\right\}, \infty\right)$,

(i) for any $k \in \mathbb{Z}$, $i$ and $x \in \mathbb{R}^{n}$,

$$
h_{i}^{k}(x)=\sum_{Q \subset Q_{i}^{k}, Q \in \mathfrak{Q}_{k}} \int_{\widehat{Q}} f * \psi_{t}(y) \theta_{t}(x-y) d y d m(t)
$$

holds true in $L^{r}\left(\mathbb{R}^{n}\right)$ and hence also in $\mathcal{S}^{\prime}\left(\mathbb{R}^{n}\right)$;

(ii) for any $k \in \mathbb{Z}$ and $i, h_{i}^{k}=\lambda_{i}^{k} a_{i}^{k}$ is a multiple of a $(p(\cdot), r, s)$-atom, where, for any $k \in$ $\mathbb{Z}$ and $i, \lambda_{i}^{k} \sim 2^{k}\left\|\mathbf{1}_{B_{i}^{k}}\right\|_{L^{p(\cdot)}\left(\mathbb{R}^{n}\right)}$ with the positive equivalence constants independent of $k$ and $i$, and $a_{i}^{k}$ is a $(p(\cdot), r, s)$-atom satisfying, for any $r \in\left(\max \left\{p_{+}, 1\right\}, \infty\right), k \in \mathbb{Z}$, $i$ and $\gamma \in \mathbb{Z}_{+}^{n}$ as in Definition 4.2(i) $)_{3}$,

$$
\begin{aligned}
& \operatorname{supp} a_{i}^{k} \subset B_{i}^{k}:=x_{Q_{i}^{k}}+B_{v\left[\ell\left(Q_{i}^{k}\right)-1\right]+u+3 \tau} \text { with } v \text { and } u \text { as in Lemma 3.3(iv), } \\
& \left\|a_{i}^{k}\right\|_{L^{r}\left(\mathbb{R}^{n}\right)} \leq\left\|\mathbf{1}_{B_{i}^{k}}\right\|_{L^{p(\cdot)}\left(\mathbb{R}^{n}\right)}^{-1}\left|B_{i}^{k}\right|^{1 / r} \text { and } \int_{\mathbb{R}^{n}} a_{i}^{k}(x) x^{\gamma} d x=0 .
\end{aligned}
$$

To show (4.10), we next consider two cases: $i \in \mathbb{N}$ and $i \in\{1, \cdots, I\}$ with some $I \in \mathbb{N}$.

Case 1) $i \in \mathbb{N}$. In this case, by [75, Lemma 4.3], to prove (4.10), it suffices to show that

$$
\lim _{l \rightarrow \infty}\left\|\sum_{l \leq|k| \leq m} \sum_{l \leq i \leq m} \lambda_{i}^{k} a_{i}^{k}\right\|_{H_{A}^{p \cdot \cdot(}\left(\mathbb{R}^{n}\right)}=0
$$

Indeed, for any $k \in \mathbb{Z}$ and $i \in \mathbb{N}$, by the fact that $\left|Q_{i}^{k} \cap \Omega_{k}\right| \geq \frac{\left|Q_{i}^{k}\right|}{2}$, we find that, for any $x \in \mathbb{R}^{n}$,

$$
M_{\mathrm{HL}}\left(\mathbf{1}_{Q_{i}^{k} \cap \Omega_{k}}\right)(x) \gtrsim \frac{1}{\left|Q_{i}^{k}\right|} \int_{Q_{i}^{k}} \mathbf{1}_{Q_{i}^{k} \cap \Omega_{k}}(y) d y \sim \frac{\left|Q_{i}^{k} \cap \Omega_{k}\right|}{\left|Q_{i}^{k}\right|} \gtrsim \frac{1}{2}
$$


where $M_{\mathrm{HL}}$ denotes the Hardy-Littlewood maximal operator as in (3.9). This, together with [75, Lemma 3.4] and Lemma 4.1, implies that, for any $l, m \in \mathbb{N}$,

$$
\begin{aligned}
& \left\|\sum_{l \leq|k| \leq m} \sum_{l \leq i \leq m}\left(2^{k} \mathbf{1}_{B_{i}^{k}}\right)^{\underline{p}}\right\|_{L^{p(\cdot) / \underline{p}}\left(\mathbb{R}^{n}\right)}^{1 / \underline{p}} \\
= & \left\|\left[\sum_{l \leq|k| \leq m} \sum_{l \leq i \leq m} 2^{k \underline{p}}\left(\mathbf{1}_{B_{i}^{k}}\right)^{2}\right]^{1 / 2}\right\|_{L^{2 p(\cdot) / \underline{p}}\left(\mathbb{R}^{n}\right)}^{2 / \underline{p}} \\
\lesssim & \left\|\left\{\sum_{l \leq|k| \leq m} \sum_{l \leq i \leq m} 2^{k \underline{p}}\left[M_{\mathrm{HL}}\left(\mathbf{1}_{Q_{i}^{k} \cap \Omega_{k}}\right)\right]^{2}\right\}^{1 / 2}\right\|_{L^{2 p(\cdot) / \underline{p}}\left(\mathbb{R}^{n}\right)}^{2 / \underline{p}} \\
\lesssim & \left\|\sum_{l \leq|k| \leq m} \sum_{l \leq i \leq m}\left(2^{k} \mathbf{1}_{Q_{i}^{k} \cap \Omega_{k}}\right)^{\underline{p}}\right\|_{L^{p(\cdot) / \underline{p}}\left(\mathbb{R}^{n}\right)}^{1 / \underline{p}},
\end{aligned}
$$

where $\underline{p}$ is defined in (4.1). In addition, from the fact that, for any $l, m \in \mathbb{N}$, $\sum_{l \leq|k| \leq m} \sum_{l \leq i \leq m} \lambda_{i}^{k} a_{i}^{k} \in H_{A}^{p(\cdot)}\left(\mathbb{R}^{n}\right)$, Theorem 4.2(i) and [75, Lemma 3.4] again, we deduce that

$$
\begin{aligned}
& \left\|\sum_{l \leq|k| \leq m} \sum_{l \leq i \leq m} \lambda_{i}^{k} a_{i}^{k}\right\|_{H_{A}^{p(\cdot)}\left(\mathbb{R}^{n}\right)} \\
& \lesssim\left\|\left\{\sum_{l \leq|k| \leq m} \sum_{l \leq i \leq m}\left[\frac{\lambda_{i}^{k} \mathbf{1}_{B_{i}^{k}}}{\left\|\mathbf{1}_{B_{i}^{k}}\right\|_{L^{p(\cdot)}\left(\mathbb{R}^{n}\right)}}\right]^{\underline{p}}\right\}^{1 / \underline{p}}\right\|_{L^{p(\cdot)\left(\mathbb{R}^{n}\right)}} \\
\sim & \left\|\left[\sum_{l \leq|k| \leq m} \sum_{l \leq i \leq m}\left(2^{k} \mathbf{1}_{B_{i}^{k}}\right)^{\underline{p}}\right]^{1 / \underline{p}}\right\|_{L^{p(\cdot)}\left(\mathbb{R}^{n}\right)} \\
\sim & \left\|\sum_{l \leq|k| \leq m} \sum_{l \leq i \leq m}\left(2^{k} \mathbf{1}_{B_{i}^{k}}\right)^{\underline{p}}\right\|_{L^{p(\cdot) / \underline{p}}\left(\mathbb{R}^{n}\right)}^{1 / \underline{p}},
\end{aligned}
$$

where $p$ is as in (4.1). On another hand, from [75, Lemma 3.4], it follows that, for any $l$, $m \in \mathbb{N}^{-}$

$$
\begin{aligned}
& \left\|\left[\sum_{l \leq|k| \leq m}\left(2^{k} \mathbf{1}_{\Omega_{k}}\right)^{\underline{p}}\right]^{1 / \underline{p}}\right\|_{L^{p(\cdot)}\left(\mathbb{R}^{n}\right)}^{\underline{p}} \\
= & \left\|\left[\sum_{l \leq|k| \leq m}\left(2^{k} \mathbf{1}_{\Omega_{k} \backslash \Omega_{k+1}}+2^{k} \mathbf{1}_{\Omega_{k+1}}\right)^{\underline{p}}\right]^{1 / \underline{p}}\right\|_{L^{p(\cdot)}\left(\mathbb{R}^{n}\right)}^{\underline{p}}
\end{aligned}
$$




$$
\lesssim\left\|\left[\sum_{l \leq|k| \leq m}\left(2^{k} \mathbf{1}_{\Omega_{k} \backslash \Omega_{k+1}}\right)^{\underline{p}}\right]^{1 / \underline{p}}\right\|_{L^{p(\cdot)}\left(\mathbb{R}^{n}\right)}^{\underline{p}}+\left(\frac{1}{2}\right)^{\underline{p}}\left\|\left[\sum_{l \leq|k| \leq m}\left(2^{k+1} \mathbf{1}_{\Omega_{k+1}}\right)^{\underline{p}}\right]^{1 / \underline{p}}\right\|_{L^{p(\cdot)}\left(\mathbb{R}^{n}\right)}^{\underline{p}} .
$$

Therefore, as $l \rightarrow \infty$, we have

$$
\left\|\left[\sum_{l \leq|k| \leq m}\left(2^{k} \mathbf{1}_{\Omega_{k}}\right)^{\underline{p}}\right]^{1 / \underline{p}}\right\|_{L^{p(\cdot)}\left(\mathbb{R}^{n}\right)} \sim\left\|\left[\sum_{l \leq|k| \leq m}\left(2^{k} \mathbf{1}_{\Omega_{k} \backslash \Omega_{k+1}}\right)^{\underline{p}}\right]^{1 / \underline{p}}\right\|_{L^{p(\cdot)}\left(\mathbb{R}^{n}\right)} .
$$

This, combined with (4.14) and (4.13), further implies that, as $l \rightarrow \infty$,

$$
\begin{aligned}
& \left\|\sum_{l \leq|k| \leq m} \sum_{l \leq i \leq m} \lambda_{i}^{k} a_{i}^{k}\right\|_{H_{A}^{p(\cdot)}\left(\mathbb{R}^{n}\right)} \\
& \sim\left\|\sum_{l \leq|k| \leq m} \sum_{l \leq i \leq m}\left(2^{k} \mathbf{1}_{Q_{i}^{k} \cap \Omega_{k}}\right)^{\underline{p}}\right\|_{L^{p(\cdot) / \underline{p}\left(\mathbb{R}^{n}\right)}}^{1 / \underline{p}} \lesssim\left\|\left[\sum_{l \leq|k| \leq m}\left(2^{k} \mathbf{1}_{\Omega_{k}}\right)^{\underline{p}}\right]^{1 / \underline{p}}\right\|_{L^{p(\cdot)}\left(\mathbb{R}^{n}\right)} \\
& \sim\left\|\left[\sum_{l \leq|k| \leq m}\left(2^{k} \mathbf{1}_{\Omega_{k} \backslash \Omega_{k+1}}\right)^{\underline{p}}\right]^{1 / \underline{p}}\right\|_{L^{p(\cdot)\left(\mathbb{R}^{n}\right)}} \sim\left\|S_{\psi}(f)\left(\sum_{l \leq|k| \leq m} \mathbf{1}_{\Omega_{k} \backslash \Omega_{k+1}}\right)^{1 / \underline{p}}\right\|_{L^{p(\cdot)}\left(\mathbb{R}^{n}\right)} \rightarrow 0 .
\end{aligned}
$$

Thus, (4.12) holds true and so does (4.10) in Case 1).

Case 2) $i \in\{1, \cdots, I\}$ with some $I \in \mathbb{N}$. In this case, to show (4.10), it suffices to prove that

$$
\lim _{l \rightarrow \infty}\left\|\sum_{l \leq|k| \leq m} \sum_{i=1}^{I} \lambda_{i}^{k} a_{i}^{k}\right\|_{H_{A}^{p(\cdot)}\left(\mathbb{R}^{n}\right)}=0 .
$$

Indeed, by a proof similar to that of (4.12), it is easy to see that (4.16) also holds true. This finishes the proof of (4.10) in Case 2) and hence of (4.10).

Step 2) In this step, we prove that

$$
f=\sum_{k \in \mathbb{Z}} \sum_{i} \lambda_{i}^{k} a_{i}^{k} \quad \text { in } \mathcal{S}^{\prime}\left(\mathbb{R}^{n}\right) .
$$

To this end, for any $x \in \mathbb{R}^{n}$, let

$$
\widetilde{f}(x):=\sum_{k \in \mathbb{Z}} \sum_{i} h_{i}^{k}(x)=\sum_{k \in \mathbb{Z}} \sum_{i} \int_{B_{k, i}} f * \psi_{t}(y) \theta_{t}(x-y) d y d m(t)
$$

in $\mathcal{S}^{\prime}\left(\mathbb{R}^{n}\right)$, where, for any $k \in \mathbb{Z}$ and $i, B_{k, i}$ is as in (4.8). Then, to show (4.17), it suffices to prove that

$$
f=\widetilde{f} \text { in } \mathcal{S}^{\prime}\left(\mathbb{R}^{n}\right)
$$


For this purpose, by the above assertion (i) and (4.7), we know that, for any given $r \in$ $\left(\max \left\{p_{+}, 1\right\}, \infty\right)$ and for any $k \in \mathbb{Z}, i$ and $x \in \mathbb{R}^{n}$,

$$
\begin{aligned}
& h_{i}^{k}(x)=\lim _{N \rightarrow \infty} \int_{0}^{\infty} \int_{\mathbb{R}^{n}} f * \psi_{t}(y) \theta_{t}(x-y) \mathbf{1}_{\substack{Q \subset Q_{i}^{k}, \in \in \mathfrak{Q}_{k} \\
|\ell(Q)| \leq N}} \widehat{Q}(y, t) d y d m(t) \\
& =\lim _{N \rightarrow \infty} \int_{\gamma(N)}^{\eta(N)} \int_{\mathbb{R}^{n}} f * \psi_{t}(y) \theta_{t}(x-y) \mathbf{1}_{B_{k, i}}(y, t) d y d m(t)
\end{aligned}
$$

holds true in $L^{r}\left(\mathbb{R}^{n}\right)$ and also in $\mathcal{S}^{\prime}\left(\mathbb{R}^{n}\right)$, where, for any $N \in \mathbb{N}, \gamma(N)$ and $\eta(N)$ are as in (3.32). For the convenience of symbols, we rewrite $\widetilde{f}$ as, for any $x \in \mathbb{R}^{n}$,

$$
\widetilde{f}(x)=\sum_{\ell \in \mathbb{N}} \int_{R^{(\ell)}} f * \psi_{t}(y) \theta_{t}(x-y) d y d m(t),
$$

where $\left\{R^{(\ell)}\right\}_{\ell \in \mathbb{N}}$ is an arbitrary permutation of $\left\{B_{k, i}\right\}_{k \in \mathbb{Z}, i}$. For any $L \in \mathbb{N}$ and $x \in \mathbb{R}^{n}$, let

$$
\widetilde{f}_{L}(x):=f(x)-\sum_{\ell=1}^{L} \int_{R^{(\ell)}} f * \psi_{t}(y) \theta_{t}(x-y) d y d m(t)
$$

Then, from (4.8), (4.9) and (4.19), it follows that, for any $L \in \mathbb{N}$ and $x \in \mathbb{R}^{n}$,

$$
\begin{aligned}
\widetilde{f}_{L}(x)= & \lim _{N \rightarrow \infty} \int_{\gamma(N)}^{\eta(N)} \int_{\mathbb{R}^{n}} f * \psi_{t}(y) \theta_{t}(x-y) \mathbf{1}_{\cup_{\ell=1}^{\infty} R^{(\ell)}}(y, t) d y d m(t) \\
& \quad-\lim _{N \rightarrow \infty} \int_{\gamma(N)}^{\eta(N)} \int_{\mathbb{R}^{n}} f * \psi_{t}(y) \theta_{t}(x-y) \mathbf{1}_{\cup_{\ell=1}^{L} R^{(\ell)}}(y, t) d y d m(t) \\
= & \lim _{N \rightarrow \infty} \int_{\gamma(N)}^{\eta(N)} \int_{\mathbb{R}^{n}} f * \psi_{t}(y) \theta_{t}(x-y) \mathbf{1}_{\cup_{\ell=L+1}^{\infty} R^{(\ell)}}(y, t) d y d m(t)
\end{aligned}
$$

holds true in $\mathcal{S}^{\prime}\left(\mathbb{R}^{n}\right)$.

Note that $H_{A}^{p(\cdot)}\left(\mathbb{R}^{n}\right)$ is continuously embedded into $\mathcal{S}^{\prime}\left(\mathbb{R}^{n}\right)$ (see [75, Lemma 4.3]). Thus, to prove (4.18), we only need to show that

$$
\left\|\widetilde{f}_{L}\right\|_{H_{A}^{p(\cdot)}\left(\mathbb{R}^{n}\right)} \rightarrow 0 \quad \text { as } L \rightarrow \infty
$$

To do this, we borrow some ideas from the proof of the atomic characterizations of $H_{A}^{p(\cdot)}\left(\mathbb{R}^{n}\right)$ (see [75, Theorem 4.8]). Indeed, for any $\varepsilon \in(0,1), L \in \mathbb{N}$ and $x \in \mathbb{R}^{n}$, let

$$
\widetilde{f}_{L}^{(\varepsilon)}(x):=\int_{\varepsilon}^{\alpha / \varepsilon} \int_{\mathbb{R}^{n}} f * \psi_{t}(y) \theta_{t}(x-y) \mathbf{1}_{\cup_{\ell=L+1}^{\infty} R^{(\ell)}}(y, t) d y d m(t)
$$


where $\alpha:=b^{-v+2(u+1)}$ with $v$ and $u$ as in Lemma 3.3(iv). Then, by the Lebesgue dominated convergence theorem, we find that, for any $\varepsilon \in(0,1), L \in \mathbb{N}$ and $x \in \mathbb{R}^{n}$,

$$
\widetilde{f}_{L}^{(\varepsilon)}(x)=\sum_{\ell=L+1}^{\infty} \int_{\mathcal{\varepsilon}}^{\alpha / \varepsilon} \int_{\mathbb{R}^{n}} f * \psi_{t}(y) \theta_{t}(x-y) \mathbf{1}_{R^{(\ell)}}(y, t) d y d m(t)=: \sum_{\ell=L+1}^{\infty} h_{\ell}^{(\varepsilon)}(x)
$$

in $\mathcal{S}^{\prime}\left(\mathbb{R}^{n}\right)$. Moreover, by some arguments similar to those used in the proofs of assertions (i) and (ii) in the proof of the sufficiency of Theorem 3.4(i) with some slight modifications, we conclude that, for any $\varepsilon \in(0,1), r \in\left(\max \left\{p_{+}, 1\right\}, \infty\right), L \in \mathbb{N}$ and $\ell \in \mathbb{N} \cap[L+1, \infty), h_{\ell}^{(\varepsilon)}$ is a multiple of a $(p(\cdot), r, s)$-atom, namely, there exist $\left\{\lambda_{\ell}\right\}_{\ell \in \mathbb{N} \cap[L+1, \infty)} \subset \mathbb{C}$ and a sequence of $(p(\cdot), r, s)$-atoms, $\left\{a_{\ell}^{(\varepsilon)}\right\}_{\ell \in \mathbb{N} \cap[L+1, \infty)}$, supported, respectively, in $\left\{B^{(\ell)}\right\}_{\ell \in \mathbb{N} \cap[L+1, \infty)} \subset \mathfrak{B}$ such that, for any $\ell \in \mathbb{N} \cap[L+1, \infty), h_{\ell}^{(\varepsilon)}=\lambda_{\ell} a_{\ell}^{(\varepsilon)}$, where, for any $\ell \in \mathbb{N} \cap[L+1, \infty), \lambda_{\ell}$ and $B^{(\ell)}$ are independent of $\varepsilon$. Therefore, for any $\varepsilon \in(0,1), L \in \mathbb{N}$ and $x \in \mathbb{R}^{n}$,

$$
\widetilde{f}_{L}^{(\varepsilon)}(x)=\sum_{\ell=L+1}^{\infty} \lambda_{\ell} a_{\ell}^{(\varepsilon)}(x) \text { in } \mathcal{S}^{\prime}\left(\mathbb{R}^{n}\right)
$$

and

$$
\left\|\left\{\sum_{\ell=L+1}^{\infty}\left[\frac{\left|\lambda_{\ell}\right| \mathbf{1}_{B^{(\ell)}}}{\left\|\mathbf{1}_{B^{(\ell)}}\right\|_{L^{p(\cdot)}\left(\mathbb{R}^{n}\right)}}\right]^{\underline{p}}\right\}^{1 / \underline{p}}\right\|_{L^{p(\cdot)}\left(\mathbb{R}^{n}\right)}<\infty
$$

On another hand, for any given

$$
N_{0} \in \mathbb{N} \cap\left[\left\lfloor\left(\frac{1}{\underline{p}}-1\right) \frac{\ln b}{\ln \lambda_{-}}\right\rfloor+2, \infty\right)
$$

with $p$ as in (4.1), let $M_{N_{0}}^{0}$ denote the radial grand maximal function as in (3.15) with $N$ replaced by $N_{0}$. Then, by the fact that, for any $\varepsilon \in(0,1)$ and $L \in \mathbb{N},\left\{a_{\ell}^{(\varepsilon)}\right\}_{\ell \in \mathbb{N} \cap[L+1, \infty)}$ is a sequence of $(p(\cdot), r, s)$-atoms and $[78,(4.8)]$, we know that, for any $\ell \in \mathbb{N} \cap[L+1, \infty)$ and $x \in \mathbb{R}^{n}$,

$$
M_{N_{0}}^{0}\left(a_{\ell}^{(\varepsilon)}\right)(x) \lesssim M_{N_{0}}^{0}\left(a_{\ell}^{(\varepsilon)}\right)(x) \mathbf{1}_{A^{\tau} B^{(\ell)}}(x)+\frac{1}{\left\|\mathbf{1}_{B^{(\ell)}}\right\|_{L^{p(\cdot)}\left(\mathbb{R}^{n}\right)}}\left[M_{\mathrm{HL}}\left(\mathbf{1}_{B^{(\ell)}}\right)(x)\right]^{\beta},
$$

where $\tau$ is as in (2.2) and

$$
\beta:=\left(\frac{\ln b}{\ln \lambda_{-}}+s+1\right) \frac{\ln \lambda_{-}}{\ln b}>\frac{1}{\underline{p}} .
$$


Moreover, since $r>1$, then, from the boundedness of $M_{\mathrm{HL}}$ on $L^{r}\left(\mathbb{R}^{n}\right)$ (see [78, Lemma 3.3(ii)]), we deduce that, for any $\varepsilon \in(0,1), L \in \mathbb{N}$ and $\ell \in \mathbb{N} \cap[L+1, \infty)$,

$$
\left\|M_{N_{0}}^{0}\left(a_{\ell}^{(\varepsilon)}\right) \mathbf{1}_{A^{\tau} B^{(\ell)}}\right\|_{L^{r}\left(\mathbb{R}^{n}\right)} \lesssim\left\|M_{\mathrm{HL}}\left(a_{\ell}^{(\varepsilon)}\right) \mathbf{1}_{A^{\tau} B^{(\ell)}}\right\|_{L^{r}\left(\mathbb{R}^{n}\right)} \lesssim \frac{\left|B^{(\ell)}\right|^{1 / r}}{\left\|\mathbf{1}_{B^{(\ell)}}\right\|_{L^{p(\cdot)}\left(\mathbb{R}^{n}\right)}},
$$

which, combined with Lemma 4.2, further implies that

$$
\begin{gathered}
\left\|\liminf _{\varepsilon \rightarrow 0^{+}}\left\{\sum_{\ell=L+1}^{\infty}\left[\left|\lambda_{\ell}\right| M_{N_{0}}^{0}\left(a_{\ell}^{(\varepsilon)}\right) \mathbf{1}_{A^{\tau} B^{(\ell)}}\right]^{\underline{p}}\right\}^{1 / \underline{p}}\right\|_{L^{p(\cdot)\left(\mathbb{R}^{n}\right)}} \\
\lesssim\left\|\left\{\sum_{\ell=L+1}^{\infty}\left[\frac{\left|\lambda_{\ell}\right| \mathbf{1}_{B^{(\ell)}}}{\left\|\mathbf{1}_{B^{(\ell)}}\right\|_{L^{p(\cdot)}\left(\mathbb{R}^{n}\right)}}\right]^{\underline{p}}\right\}^{1 / \underline{p}}\right\|_{L^{p(\cdot)\left(\mathbb{R}^{n}\right)}} \cdot
\end{gathered}
$$

In addition, let $\varepsilon:=\gamma(N)$ with $N \in \mathbb{N} \cap\left[\left\lfloor\frac{-u-1}{v}\right\rfloor+1, \infty\right)$. Then, by (4.20), we find that

$$
M_{N_{0}}^{0}\left(\widetilde{f}_{L}\right)=M_{N_{0}}^{0}\left(\lim _{N \rightarrow \infty} \widetilde{f}_{L}^{(\gamma(N))}\right) \leq \liminf _{N \rightarrow \infty} M_{N_{0}}^{0}\left(\widetilde{f}_{L}^{(\gamma(N))}\right) .
$$

From this, [12, p. 17, Proposition 3.10], (4.22) and (4.24), it follows that, for any $L \in \mathbb{N}$,

$$
\begin{aligned}
\left\|\widetilde{f}_{L}\right\|_{H_{A}^{p(\cdot)}\left(\mathbb{R}^{n}\right)} \leq & \left\|\liminf _{N \rightarrow \infty} M_{N_{0}}^{0}\left(\widetilde{f}_{L}^{(\gamma(N))}\right)\right\|_{L^{p(\cdot)}\left(\mathbb{R}^{n}\right)} \\
\leq & \left\|\liminf _{N \rightarrow \infty} \sum_{\ell=L+1}^{\infty}\left|\lambda_{\ell}\right| M_{N_{0}}^{0}\left(a_{\ell}^{(\gamma(N))}\right)\right\|_{L^{p(\cdot)}\left(\mathbb{R}^{n}\right)} \\
& \lesssim\left\|\liminf _{N \rightarrow \infty} \sum_{\ell=L+1}^{\infty}\left|\lambda_{\ell}\right| M_{N_{0}}^{0}\left(a_{\ell}^{(\gamma(N))}\right) \mathbf{1}_{A^{\tau} B^{(\ell)}}\right\|_{L^{p(\cdot)}\left(\mathbb{R}^{n}\right)} \\
& +\| \sum_{\ell=L+1}^{\infty} \frac{\left|\lambda_{\ell}\right|}{\left\|\mathbf{1}_{A^{\tau} B^{(\ell)}}\right\|_{L^{p(\cdot)}\left(\mathbb{R}^{n}\right)}}\left[M_{\mathrm{HL}}\left(\mathbf{1}_{\left.B^{(\ell)}\right)}\right]^{\beta} \|_{L^{p(\cdot)}\left(\mathbb{R}^{n}\right)} .\right.
\end{aligned}
$$

This, together with (4.26), [75, Lemma 3.4], Lemma 4.1, (4.25), further implies that, for any $L \in \mathbb{N}$,

$$
\begin{aligned}
\left\|\widetilde{f}_{L}\right\|_{H_{A}^{p(\cdot)}\left(\mathbb{R}^{n}\right)} \lesssim\left\|\liminf _{N \rightarrow \infty}\left\{\sum_{\ell=L+1}^{\infty}\left[\left|\lambda_{\ell}\right| M_{N_{0}}^{0}\left(a_{\ell}^{(\gamma(N))}\right) \mathbf{1}_{A^{\tau} B^{(\ell)}}\right]^{\underline{p}}\right\}^{1 / \underline{p}}\right\|_{L^{p(\cdot)\left(\mathbb{R}^{n}\right)}} \\
+\|\left\{\sum_{\ell=L+1}^{\infty} \frac{\left|\lambda_{\ell}\right|}{\left\|\mathbf{1}_{B^{(\ell)}}\right\|_{L^{p(\cdot)}\left(\mathbb{R}^{n}\right)}}\left[M_{\mathrm{HL}}\left(\mathbf{1}_{\left.B^{(\ell)}\right)}\right]^{\beta}\right\}^{1 / \beta} \|_{L^{\beta p(\cdot)}\left(\mathbb{R}^{n}\right)}^{\beta}\right. \\
\lesssim\left\|\left\{\sum_{\ell=L+1}^{\infty}\left[\frac{\left|\lambda_{\ell}\right| \mathbf{1}_{B^{(\ell)}}}{\left\|\mathbf{1}_{B^{(\ell)}}\right\|_{L^{p(\cdot)}\left(\mathbb{R}^{n}\right)}}\right]^{\underline{p}}\right\}^{1 / \underline{p}}\right\|_{L^{p(\cdot)}\left(\mathbb{R}^{n}\right)} \cdot
\end{aligned}
$$


By this and (4.23), we know that (4.21) holds true, which completes the proof of (4.17).

Step 3) By (4.17), Theorem 4.2(i) and some arguments similar to those used in the estimations of both (4.13) and (4.15), we conclude that

$$
\begin{aligned}
\|f\|_{H_{A}^{p(\cdot)}\left(\mathbb{R}^{n}\right)} & \sim\left\|\left\{\sum_{k \in \mathbb{Z}} \sum_{i}\left[\frac{\lambda_{i}^{k} \mathbf{1}_{B_{i}^{k}}}{\left\|\mathbf{1}_{B_{i}^{k}}\right\|_{L^{p(\cdot)}\left(\mathbb{R}^{n}\right)}}\right]^{\underline{p}}\right\}^{1 / \underline{p}}\right\| \\
& \sim\left\|\left[\sum_{k \in \mathbb{Z}} \sum_{i}\left(2^{k} \mathbf{1}_{B_{i}^{k}}\right)^{\underline{p}}\right]^{1 / \underline{p}}\right\|_{L^{p(\cdot)}\left(\mathbb{R}^{n}\right)} \lesssim\left\|\sum_{L^{p(\cdot)}\left(\mathbb{R}^{n}\right)} \sum_{i}\left(2^{k} \mathbf{1}_{Q_{i}^{k} \cap \Omega_{k}}\right)^{\underline{p}}\right\|_{L^{p(\cdot) / \underline{p}}\left(\mathbb{R}^{n}\right)}^{1 / \underline{p}} \\
& \left.\lesssim\left\|\left[\sum_{k \in \mathbb{Z}}\left(2^{k} \mathbf{1}_{\Omega_{k}}\right)^{\underline{p}}\right]^{1 / \underline{p}}\right\|_{L^{p(\cdot)}\left(\mathbb{R}^{n}\right)} \sim \| \sum_{k \in \mathbb{Z}}\left(2^{k} \mathbf{1}_{\Omega_{k} \backslash \Omega_{k+1}}\right)^{\underline{p}}\right]^{1 / \underline{p}} \|_{L^{p(\cdot)}\left(\mathbb{R}^{n}\right)} \\
& \lesssim\left\|S_{\psi}(f)\left[\sum_{k \in \mathbb{Z}} \mathbf{1}_{\Omega_{k} \backslash \Omega_{k+1}}\right]^{1 / \underline{p}}\right\|_{L^{p(\cdot)}\left(\mathbb{R}^{n}\right)} \sim\left\|S_{\psi}(f)\right\|_{L^{p(\cdot)}\left(\mathbb{R}^{n}\right)}
\end{aligned}
$$

which implies that $f \in H_{A}^{p(\cdot)}\left(\mathbb{R}^{n}\right)$ and (4.6) holds true. This finishes the proof of the sufficiency of Theorem 4.4(i).

Remark 4.2. Let us point out that, in the original proof of the sufficiency of Theorem 4.4(i) (namely, [75, Theorem 6.1]), the method used therein is the same as that used in the proof of the sufficiency of [79, Theorem 2.7]; see [75, p. 1199]. Thus,

(i) similarly to Remark 3.2, the following fact is needed: for any $f \in \mathcal{S}_{0}^{\prime}\left(\mathbb{R}^{n}\right)$, the $L^{p(\cdot)}\left(\mathbb{R}^{n}\right)$ quasi-norms of the anisotropic Lusin area function $S(f)$ are independent of the choices of $\theta$ and $\psi$ as in Lemma 3.1. However, in the original proof of the sufficiency of [75, Theorem 6.1]), the authors did not give the proof of this necessary fact. In the present article, we seal this gap in Theorem 4.3 above;

(ii) similarly to Remark 3.3, both the equalities (3.39) and (3.40) are also used in the original proof of the sufficiency of Theorem 4.4(i) (namely, [75, Theorem 6.1]). However, the authors therein did not prove these two equalities. In the present article, we also seal these gaps existing in the original proof of the sufficiency of [75, Theorem 6.1]; see, respectively, the proofs of (4.18) and (4.11) above for the details.

\subsection{Some applications}

In this subsection, we give some applications for the anisotropic summability of Fourier transforms introduced in [76]. 
Let $f \in L^{p}\left(\mathbb{R}^{n}\right)$ for some $p \in[1,2]$. Recall that the Fourier inversion formula, namely, for any $x \in \mathbb{R}^{n}$,

$$
f(x):=\int_{\mathbb{R}^{n}} \widehat{f}(t) e^{2 \pi \imath x \cdot t} d t
$$

holds true if $\widehat{f} \in L^{1}\left(\mathbb{R}^{n}\right)$, where $\widehat{f}$ denotes the Fourier transform of $f$ as in (3.7). This motivates the following definition of summability. We always assume that

$$
\theta \in C_{0}(\mathbb{R}), \quad \theta(|\cdot|) \in L^{1}\left(\mathbb{R}^{n}\right), \quad \theta(0)=1 \text { and } \theta \text { is even, }
$$

where $C_{0}(\mathbb{R})$ denotes the set of all continuous functions $f$ on $\mathbb{R}$ satisfying that $\lim _{|x| \rightarrow \infty} f(x)=0$. Let $A^{*}$ be the adjoint matrix of $A$. The $m$-th anisotropic $\theta$-mean of the function $f \in L^{p}\left(\mathbb{R}^{n}\right)$, with $p \in[1,2]$, is defined by setting, for any $m \in \mathbb{Z}$ and $x \in \mathbb{R}^{n}$,

$$
\sigma_{m}^{\theta} f(x):=\int_{\mathbb{R}^{n}} \theta\left(\left|\left(A^{*}\right)^{-m} u\right|\right) \widehat{f}(u) e^{2 \pi i x \cdot u} d u .
$$

Let $\theta_{0}(x):=\theta(|x|)$ for any $x \in \mathbb{R}^{n}$ and assume that

$$
\widehat{\theta_{0}} \in L^{1}\left(\mathbb{R}^{n}\right) .
$$

It was proved in [76] that, for any $m \in \mathbb{Z}, f \in L^{1}\left(\mathbb{R}^{n}\right)$ and $x \in \mathbb{R}^{n}$, we can rewrite $\sigma_{m}^{\theta} f$ as

$$
\sigma_{m}^{\theta} f(x)=b^{m} \int_{\mathbb{R}^{n}} f(t) \widehat{\theta_{0}}\left(A^{m}(x-t)\right) d t .
$$

Moreover, we can extend the definition of the anisotropic $\theta$-means to any $f \in L^{p(\cdot)}\left(\mathbb{R}^{n}\right)$ with $p_{-} \in[1, \infty)$ by setting, for any $x \in \mathbb{R}^{n}$,

$$
\sigma_{m}^{\theta} f(x):=b^{m} \int_{\mathbb{R}^{n}} f(x-t) \widehat{\theta_{0}}\left(A^{m} t\right) d t .
$$

Then we define the maximal $\theta$-operator $\sigma_{*}^{\theta}$ by setting, for any $f \in L^{p(\cdot)}\left(\mathbb{R}^{n}\right)$ with $p_{-} \in$ $[1, \infty)$,

$$
\sigma_{*}^{\theta} f:=\sup _{m \in \mathbb{Z}}\left|\sigma_{m}^{\theta} f\right|
$$

The following boundedness of maximal $\theta$-operators from $H_{A}^{p(\cdot)}\left(\mathbb{R}^{n}\right)$ to $L^{p(\cdot)}\left(\mathbb{R}^{n}\right)$ is just [75, Theorem 7.4].

Theorem 4.5. Let $\theta$ and $\theta_{0}$ be, respectively, as in (4.27) and (4.28) satisfying that there exists a constant $\beta \in(1, \infty)$ such that, for any $\alpha \in \mathbb{Z}_{+}^{n}$ and $x \in \mathbb{R}^{n} \backslash\left\{\overrightarrow{0}_{n}\right\}$,

$$
\left|\partial^{\alpha} \widehat{\theta_{0}}(x)\right| \leq C_{(\alpha, \beta)}|x|^{-\beta},
$$


where $C_{(\alpha, \beta)}$ is a positive constant independent of $x$. If $p(\cdot) \in C^{\log }\left(\mathbb{R}^{n}\right)$,

$$
\beta \in\left(\frac{\ln b}{\ln \lambda_{-}}, \infty\right) \text { and } p_{-} \in\left(\frac{\ln b}{\beta \ln \lambda_{-}}, \infty\right),
$$

then there exists a positive constant $C_{\left(p_{-}, p_{+}\right)}$, with $p_{-}$and $p_{+}$as in (4.1), such that, for any $f \in H_{A}^{p(\cdot)}\left(\mathbb{R}^{n}\right)$,

$$
\left\|\sigma_{*}^{\theta} f\right\|_{L^{p(\cdot)\left(\mathbb{R}^{n}\right)}} \leq C_{\left(p_{-,} p_{+}\right)}\|f\|_{H_{A}^{p(\cdot)}\left(\mathbb{R}^{n}\right)} .
$$

Remark 4.3. If $A:=d \mathrm{I}_{n \times n}$ for some $d \in \mathbb{R}$ with $|d| \in(1, \infty)$, then $\frac{\ln b}{\ln \lambda_{-}}=n$ and Theorem 4.5 goes back to the classical result with $\beta \in(n, \infty)$ and $p \in(n / \beta, \infty)$ (see Weisz [111]). The classical result was proved in a special case, namely, for the Bochner-Riesz means, in Stein et al. [97]. For the same case, a counterexample was also given in [97] to show that the same conclusion is not true for $p \in(0, n / \beta]$.

Corollary 4.1. Let all the assumptions be the same as in Theorem 4.5.

(i) If $f \in H_{A}^{p(\cdot)}\left(\mathbb{R}^{n}\right)$, then $\sigma_{m}^{\theta} f$ converges pointwisely almost everywhere as well as in the $L^{p(\cdot)}\left(\mathbb{R}^{n}\right)$ quasi-norm as $m \rightarrow \infty$;

(ii) If $f \in H_{A}^{p(\cdot)}\left(\mathbb{R}^{n}\right)$ and there exists a subset $I \subset \mathbb{R}^{n}$ such that the restriction $\left.f\right|_{I} \in L^{r(\cdot)}(I)$ with $r_{-} \in[1, \infty)$, then

$$
\lim _{m \rightarrow \infty} \sigma_{m}^{\theta} f(x)=f(x)
$$

pointwisely for almost every $x \in I$ as well as in the $L^{p(\cdot)}(I)$ quasi-norm;

(iii) If $f \in L^{p(\cdot)}\left(\mathbb{R}^{n}\right)$ with $p_{-} \in(1, \infty)$, then

$$
\lim _{m \rightarrow \infty} \sigma_{m}^{\theta} f(x)=f(x)
$$

pointwisely for almost every $x \in \mathbb{R}^{n}$ as well as in the $L^{p(\cdot)}\left(\mathbb{R}^{n}\right)$ norm.

Remark 4.4. Corollary 4.1(iii) for the Bochner-Riesz means in the classical case (namely, when $p(\cdot)=$ a constant $\in(0, \infty)$ and $A:=d \mathrm{I}_{n \times n}$ for some $d \in \mathbb{R}$ with $\left.|d| \in(1, \infty)\right)$ can be found in Stein et al. [97] and Weisz [111].

Let $\alpha \in(0, \infty)$ and $\gamma \in \mathbb{N}$. Recall also that the Bochner-Riesz summation and the Weierstrass summation are, respectively, defined by setting, for any $t \in \mathbb{R}^{n}$,

$$
\theta_{0}(t):= \begin{cases}\left(1-|t|^{\gamma}\right)^{\alpha}, & \text { when }|t| \in[0,1) \\ 0, & \text { when }|t| \in[1, \infty)\end{cases}
$$

and

$$
\theta_{0}(t):=e^{-|t|^{2} / 2}
$$

As special cases of Theorem 4.5, we further obtain the following conclusions (see [75, Theorems 7.10 and 7.11]). 
Theorem 4.6. Let $p(\cdot) \in C^{\log }\left(\mathbb{R}^{n}\right)$.

(i) If $\theta_{0}$ is as in (4.30) with

$$
\alpha \in\left(\max \left\{\frac{n-1}{2}, \frac{\ln b}{\ln \lambda_{-}}-\frac{n+1}{2}\right\}, \infty\right) \text { and } p_{-} \in\left(\frac{\ln b}{\ln \lambda_{-}(n / 2+\alpha+1 / 2)}, \infty\right),
$$

then there exists a positive constant $C_{\left(p_{-}, p_{+}\right)}$, with $p_{-}$and $p_{+}$as in (4.1), such that, for any $f \in H_{A}^{p(\cdot)}\left(\mathbb{R}^{n}\right)$,

$$
\left\|\sigma_{*}^{\theta} f\right\|_{L^{p(\cdot)}\left(\mathbb{R}^{n}\right)} \leq C_{\left(p_{-,} p_{+}\right)}\|f\|_{H_{A}^{p(\cdot)}\left(\mathbb{R}^{n}\right)^{\prime}}
$$

(ii) If $\theta_{0}$ is as in (4.31), then (4.32) also holds true for any $f \in H_{A}^{p(\cdot)}\left(\mathbb{R}^{n}\right)$ with $p_{-} \in(0, \infty)$.

Remark 4.5. (i) Let $\theta_{0}$ be as in (4.30) or (4.31). Then the corresponding conclusions in Corollaries 4.1 hold true as well.

(ii) We point out that the dual spaces and the characterizations, respectively, in terms of wavelets and molecules, of the anisotropic variable Hardy space $H_{A}^{p(\cdot)}\left(\mathbb{R}^{n}\right)$ are still unknown. In addition, it is an interesting question to consider the boundedness of Calderón-Zygmund operators on $H_{A}^{p(\cdot)}\left(\mathbb{R}^{n}\right)$.

(iii) Recall that Wang [110] obtained a multiplier theorem on anisotropic Hardy spaces $H_{A}^{p}\left(\mathbb{R}^{n}\right)$; however, the corresponding conclusion on the space $H_{A}^{p(\cdot)}\left(\mathbb{R}^{n}\right)$ is still unclear.

\section{Anisotropic variable Hardy-Lorentz spaces}

In this section, we present the real-variable theory of anisotropic variable Hardy-Lorentz spaces from $[76,78]$. To do so, we first recall the following notion of variable Lorentz spaces $L^{p(\cdot), q}\left(\mathbb{R}^{n}\right)$, which are known as special cases of the variable Lorentz spaces $L^{p(\cdot), q(\cdot)}\left(\mathbb{R}^{n}\right)$ investigated by Kempka and Vybíral in [59].

Definition 5.1. Let $p(\cdot) \in \mathcal{P}\left(\mathbb{R}^{n}\right)$. The variable Lorentz space $L^{p(\cdot), q}\left(\mathbb{R}^{n}\right)$ is defined to be the set of all measurable functions $f$ such that

$$
\|f\|_{L^{p(\cdot), q\left(\mathbb{R}^{n}\right)}}:= \begin{cases}{\left[\int_{0}^{\infty} \lambda^{q}\left\|\mathbf{1}_{\left\{x \in \mathbb{R}^{n}:|f(x)|>\lambda\right\}}\right\|_{L^{p(\cdot)\left(\mathbb{R}^{n}\right)}}^{q} \frac{d \lambda}{\lambda}\right]^{1 / q},} & \text { when } q \in(0, \infty), \\ \sup _{\lambda \in(0, \infty)}\left[\lambda\left\|\mathbf{1}_{\left\{x \in \mathbb{R}^{n}:|f(x)|>\lambda\right\}}\right\|_{L^{p(\cdot)}\left(\mathbb{R}^{n}\right)}\right], & \text { when } q=\infty,\end{cases}
$$

is finite. 
Moreover, in [78, Definition 2.10], the authors introduced anisotropic variable HardyLorentz spaces as follows.

Definition 5.2. Let $p(\cdot) \in C^{\log }\left(\mathbb{R}^{n}\right), q \in(0, \infty]$ and $N$ be as in (4.4). The anisotropic variable Hardy-Lorentz space $H_{A}^{p(\cdot), q}\left(\mathbb{R}^{n}\right)$ is defined by setting

$$
H_{A}^{p(\cdot), q}\left(\mathbb{R}^{n}\right):=\left\{f \in \mathcal{S}^{\prime}\left(\mathbb{R}^{n}\right): M_{N}^{0}(f) \in L^{p(\cdot), q}\left(\mathbb{R}^{n}\right)\right\}
$$

and, for any $f \in H_{A}^{p(\cdot), q}\left(\mathbb{R}^{n}\right)$, let

$$
\|f\|_{H_{A}^{p(\cdot), q}\left(\mathbb{R}^{n}\right)}:=\left\|M_{N}^{0}(f)\right\|_{L^{p(\cdot), q\left(\mathbb{R}^{n}\right)}}
$$

where $M_{N}^{0}(f)$ denotes the radial grand maximal function as in (3.15).

Remark 5.1. (i) Even though the quasi-norm of $H_{A}^{p(\cdot), q}\left(\mathbb{R}^{n}\right)$ in Definition 5.2 depends on $N$, it follows from Theorem 5.1 below that the space $H_{A}^{p(\cdot), q}\left(\mathbb{R}^{n}\right)$ is independent of the choice of $N$ as long as $N$ is as in (4.4). If $p(\cdot) \equiv p \in(0, \infty)$, then the space $H_{A}^{p(\cdot), q}\left(\mathbb{R}^{n}\right)$ is just the anisotropic Hardy-Lorentz space $H_{A}^{p, q}\left(\mathbb{R}^{n}\right)$ investigated by Liu et al. in [77] (see also Definition 3.2) and, if $A:=d \mathrm{I}_{n \times n}$ for some $d \in \mathbb{R}$ with $|d| \in(1, \infty)$ and $q=\infty$, then the space $H_{A}^{p(\cdot), \infty}\left(\mathbb{R}^{n}\right)$ becomes the variable weak Hardy space introduced by Yan et al. in [114].

(ii) Very recently, via the variable Lorentz spaces $\mathcal{L}^{p(\cdot), q(\cdot)}\left(\mathbb{R}^{n}\right)$ in [31], where

$$
p(\cdot), q(\cdot):(0, \infty) \rightarrow(0, \infty)
$$

are bounded measurable functions, Almeida et al. [2] investigated another sort of anisotropic variable Hardy-Lorentz spaces $H^{p(\cdot), q(\cdot)}\left(\mathbb{R}^{n}, A\right)$. As was mentioned in [59, Remark 2.6], the space $\mathcal{L}^{p(\cdot), q(\cdot)}\left(\mathbb{R}^{n}\right)$ in [31] never coincides with the space $L^{p(\cdot)}\left(\mathbb{R}^{n}\right)$, because the variable exponent $p(\cdot)$ in $\mathcal{L}^{p(\cdot), q(\cdot)}\left(\mathbb{R}^{n}\right)$ is only defined on $(0, \infty)$ while not on $\mathbb{R}^{n}$. On another hand, the space $H_{A}^{p(\cdot), q}\left(\mathbb{R}^{n}\right)$, in Definition 5.2, is defined via the variable Lorentz space $L^{p(\cdot), q(\cdot)}\left(\mathbb{R}^{n}\right)$ (with $q(\cdot) \equiv$ a constant $\epsilon$ $(0, \infty])$ from [59], which is not covered by the space $H^{p(\cdot), q(\cdot)}\left(\mathbb{R}^{n}, A\right)$ in [2]. Moreover, as was pointed out in [2, p. 6], the key tool of [2] is the fact that the set $L_{\text {loc }}^{1}\left(\mathbb{R}^{n}\right) \cap H^{p(\cdot), q(\cdot)}\left(\mathbb{R}^{n}, A\right)$ is dense in $H^{p(\cdot), q(\cdot)}\left(\mathbb{R}^{n}, A\right)$. Therefore, the method used in [2] does not work for $H_{A}^{p(\cdot), q}\left(\mathbb{R}^{n}\right)$ in [78], due to the lack of a dense function subspace of $H_{A}^{p(\cdot), \infty}\left(\mathbb{R}^{n}\right)$ even when $p(\cdot) \equiv$ a constant $\in(0, \infty)$ and $A:=d \mathrm{I}_{n \times n}$ for some $d \in \mathbb{R}$ with $|d| \in(1, \infty)$.

\subsection{Several equivalent characterizations of $H_{A}^{p(\cdot), q}\left(\mathbb{R}^{n}\right)$}

The following radial or non-tangential maximal function characterizations of $H_{A}^{p(\cdot), q}\left(\mathbb{R}^{n}\right)$ are established in [78, Theorem 3.8]. 
Theorem 5.1. Suppose that $p(\cdot) \in C^{\log }\left(\mathbb{R}^{n}\right)$ and $\phi \in \mathcal{S}\left(\mathbb{R}^{n}\right)$ satisfying $\int_{\mathbb{R}^{n}} \phi(x) d x \neq 0$. Then, for any $f \in \mathcal{S}^{\prime}\left(\mathbb{R}^{n}\right)$, the following statements are mutually equivalent:

(i) $f \in H_{A}^{p(\cdot), q}\left(\mathbb{R}^{n}\right)$;

(ii) $M_{\phi}(f) \in L^{p(\cdot), q}\left(\mathbb{R}^{n}\right)$;

(iii) $M_{\phi}^{0}(f) \in L^{p(\cdot), q}\left(\mathbb{R}^{n}\right)$,

where $M_{\phi}(f)$ and $M_{\phi}^{0}(f)$ are, respectively, as in (3.3) and (3.6). Moreover, there exist two positive constants $C$ and $\widetilde{C}$, independent of $f$, such that

$$
\|f\|_{H_{A}^{p(\cdot), q}\left(\mathbb{R}^{n}\right)} \leq C\left\|M_{\phi}^{0}(f)\right\|_{L^{p(\cdot), q\left(\mathbb{R}^{n}\right)}} \leq C\left\|M_{\phi}(f)\right\|_{L^{p(\cdot), q\left(\mathbb{R}^{n}\right)}} \leq \widetilde{C}\|f\|_{H_{A}^{p(\cdot), q}\left(\mathbb{R}^{n}\right)} .
$$

Definition 5.3. Let $p(\cdot) \in C^{\log }\left(\mathbb{R}^{n}\right), q \in(0, \infty], r \in(1, \infty]$ and $s$ be as in (4.5).

(i) The anisotropic variable atomic Hardy-Lorentz space $H_{A}^{p(\cdot), r, s, q}\left(\mathbb{R}^{n}\right)$ is defined to be the set of all $f \in \mathcal{S}^{\prime}\left(\mathbb{R}^{n}\right)$ satisfying that there exist a sequence of $(p(\cdot), r, s)$-atoms,

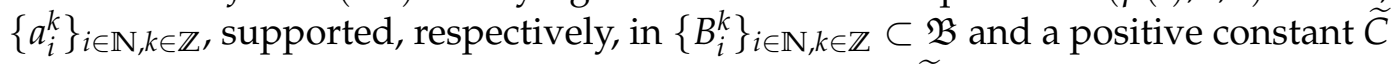
such that, for any $x \in \mathbb{R}^{n}$ and $k \in \mathbb{Z}, \sum_{i \in \mathbb{N}} \mathbf{1}_{A^{j_{0}} B_{i}^{k}}(x) \leq \widetilde{C}$ with some $j_{0} \in \mathbb{Z} \backslash \mathbb{N}$, and

$$
f=\sum_{k \in \mathbb{Z}} \sum_{i \in \mathbb{N}} \lambda_{i}^{k} a_{i}^{k} \quad \text { in } \mathcal{S}^{\prime}\left(\mathbb{R}^{n}\right),
$$

where $\lambda_{i}^{k} \sim 2^{k}\left\|\mathbf{1}_{B_{i}^{k}}\right\|_{L^{p(\cdot)}\left(\mathbb{R}^{n}\right)}$ for any $k \in \mathbb{Z}$ and $i \in \mathbb{N}$ with the positive equivalence constants independent of $k$ and $i$. Moreover, for any $f \in H_{A}^{p(\cdot), r, s, q}\left(\mathbb{R}^{n}\right)$, let

$$
\|f\|_{H_{A}^{p(\cdot), r, q, q}\left(\mathbb{R}^{n}\right)}:=\inf \left[\sum_{k \in \mathbb{Z}}\left\|\left\{\sum_{i \in \mathbb{N}}\left[\frac{\lambda_{i}^{k} \mathbf{1}_{B_{i}^{k}}}{\left\|\mathbf{1}_{B_{i}^{k}}\right\|_{L^{p(\cdot)}\left(\mathbb{R}^{n}\right)}}\right]^{\underline{p}}\right\}^{1 / \underline{p}}\right\|_{L^{p(\cdot)}\left(\mathbb{R}^{n)}\right.}^{q}\right]^{1 / q}
$$

with the usual modification made when $q=\infty$, where the infimum is taken over all the decompositions of $f$ as above.

(ii) The anisotropic variable finite atomic Hardy-Lorentz space $H_{A, f i n}^{p(\cdot), r, q}\left(\mathbb{R}^{n}\right)$ is defined to be the set of all $f \in \mathcal{S}^{\prime}\left(\mathbb{R}^{n}\right)$ satisfying that there exist $K, I \in \mathbb{N}$, a finite sequence of $(p(\cdot), r, s)$-atoms, $\left\{a_{i}^{k}\right\}_{i \in[1, I] \cap \mathbb{N}, k \in[1, K] \cap \mathbb{N}}$, supported, respectively, in $\left\{B_{i}^{k}\right\}_{i \in[1, I] \cap \mathbb{N}, k \in[1, K] \cap \mathbb{N}} \subset \mathfrak{B}$ and a positive constant $\widetilde{C}$, independent of $I$ and $K$, such that $\sum_{i=1}^{I} \mathbf{1}_{A^{j} B_{i}^{k}}(x) \leq \widetilde{C}$ for any $x \in \mathbb{R}^{n}$ and $k \in[1, K] \cap \mathbb{N}$, with some $j_{0} \in \mathbb{Z} \backslash \mathbb{N}$, and

$$
f=\sum_{k=1}^{K} \sum_{i=1}^{I} \lambda_{i}^{k} a_{i}^{k} \quad \text { in } \mathcal{S}^{\prime}\left(\mathbb{R}^{n}\right)
$$


where, for any $k \in[1, K] \cap \mathbb{N}$ and $i \in[1, I] \cap \mathbb{N}, \lambda_{i}^{k} \sim 2^{k}\left\|\mathbf{1}_{B_{i}^{k}}\right\|_{L^{p(\cdot)}\left(\mathbb{R}^{n}\right)}$ with the positive equivalence constants independent of $k, K, i$ and $I$. Moreover, for any $f \in H_{A, \text { fin }}^{p(\cdot), s, q}\left(\mathbb{R}^{n}\right)$, let

$$
\|f\|_{H_{A, \text { fin }}^{p(\cdot, r, q, q}\left(\mathbb{R}^{n}\right)}:=\inf \left[\sum_{k=1}^{K}\left\|\left\{\sum_{i=1}^{I}\left[\frac{\lambda_{i}^{k} \mathbf{1}_{B_{i}^{k}}}{\left\|\mathbf{1}_{B_{i}^{k}}\right\|_{L^{p(\cdot)}\left(\mathbb{R}^{n}\right)}}\right]^{\underline{p}}\right\}^{1 / \underline{p}}\right\|_{L^{p(\cdot)}\left(\mathbb{R}^{n}\right)}^{q}\right]^{\frac{1}{q}}
$$

with the usual modification made when $q=\infty$, where the infimum is taken over all the decompositions of $f$ as above.

The following conclusions are just [78, Theorem 4.8] and [76, Theorem 2.14].

Theorem 5.2. Let $p(\cdot) \in C^{\log }\left(\mathbb{R}^{n}\right), q \in(0, \infty]$ and $s$ be as in (4.5) and $r \in\left(\max \left\{p_{+}, 1\right\}, \infty\right]$ with $p_{+}$as in (4.1). Then

(i) $H_{A}^{p(\cdot), q}\left(\mathbb{R}^{n}\right)=H_{A}^{p(\cdot), r, s, q}\left(\mathbb{R}^{n}\right)$ with equivalent quasi-norms;

(ii) $\|\cdot\|_{H_{A, \text { fin }}^{p(\cdot, r, q}\left(\mathbb{R}^{n}\right)}$ and $\|\cdot\|_{H_{A}^{p(\cdot), q}\left(\mathbb{R}^{n}\right)}$ are equivalent quasi-norms on $H_{A, \text { fin }}^{p(\cdot), r, q, q}\left(\mathbb{R}^{n}\right)$ for each $r \in\left(\max \left\{p_{+}, 1\right\}, \infty\right)$;

(iii) $\|\cdot\|_{H_{A, \text { fin }}^{p(\cdot, \infty, q, q}\left(\mathbb{R}^{n}\right)}$ and $\|\cdot\|_{H_{A}^{p(\cdot), q}\left(\mathbb{R}^{n}\right)}$ are equivalent quasi-norms on $H_{A, \text { fin }}^{p(\cdot), s, s, q}\left(\mathbb{R}^{n}\right) \cap C\left(\mathbb{R}^{n}\right)$.

\subsection{Littlewood-Paley function characterizations of $H_{A}^{p(\cdot), q}\left(\mathbb{R}^{n}\right)$}

In this subsection, we display the characterizations of $H_{A}^{p(\cdot), q}\left(\mathbb{R}^{n}\right)$ in terms of the anisotropic Lusin area function, the anisotropic Littlewood-Paley $g$-function or $g_{\lambda}^{*}$ function; see [76,78]. Meanwhile, we also correct some errors existing in the proof of the sufficiency of [78, Theorem 5.2], namely, the Lusin area function characterizations of $H_{A}^{p(\cdot), q}\left(\mathbb{R}^{n}\right)$.

The following Fefferman-Stein vector-valued inequality of the maximal operator $M_{\mathrm{HL}}$ on the variable Lorentz space $L^{p(\cdot), q}\left(\mathbb{R}^{n}\right)$ is just [76, Lemma 3.5].

Lemma 5.1. Let $r \in(1, \infty]$. Assume that $p(\cdot) \in C^{\log }\left(\mathbb{R}^{n}\right)$ satisfies $1<p_{-} \leq p_{+}<\infty$. Then there exists a positive constant $C$ such that, for any sequence $\left\{f_{k}\right\}_{k \in \mathbb{N}}$ of measurable functions,

$$
\left\|\left\{\sum_{k \in \mathbb{N}}\left[M_{\mathrm{HL}}\left(f_{k}\right)\right]^{r}\right\}^{1 / r}\right\|_{L^{p(\cdot), q\left(\mathbb{R}^{n}\right)}} \leq C\left\|\left(\sum_{k \in \mathbb{N}}\left|f_{k}\right|^{r}\right)^{1 / r}\right\|_{L^{p(\cdot), q\left(\mathbb{R}^{n}\right)}}
$$

with the usual modification made when $r=\infty$, where $M_{\mathrm{HL}}$ denotes the Hardy-Littlewood maximal operator as in (3.9). 
Via Lemmas 5.1 and 3.4 as well as a proof similar to that of Theorem 3.5, we have the following conclusion; the details are omitted.

Theorem 5.3. Let $p(\cdot) \in C^{\log }\left(\mathbb{R}^{n}\right)$ and $\theta, \psi$ be as in Lemma 3.1 with $s$ as in (4.5). Then there exists a positive constant $C$ such that, for any $f \in \mathcal{S}_{0}^{\prime}\left(\mathbb{R}^{n}\right)$,

$$
\frac{1}{C}\left\|S_{\theta}(f)\right\|_{L^{p(\cdot), q\left(\mathbb{R}^{n}\right)}} \leq\left\|S_{\psi}(f)\right\|_{L^{p(\cdot), q\left(\mathbb{R}^{n}\right)}} \leq C\left\|S_{\theta}(f)\right\|_{L^{p(\cdot), q\left(\mathbb{R}^{n}\right)}},
$$

where $S_{\theta}(f)$ and $S_{\psi}(f)$ are the anisotropic Lusin area functions as in (3.8) defined, respectively, via $\theta$ and $\psi$.

In [78, Theorem 5.2] and [76, Theorems 2.9 and 2.10], the authors established the characterizations of the space $H_{A}^{p(\cdot), q}\left(\mathbb{R}^{n}\right)$, respectively, in terms of the anisotropic Lusin area function, the anisotropic Littlewood-Paley $g$-function or $g_{\lambda}^{*}$-function as follows.

Theorem 5.4. Let $p(\cdot) \in C^{\log }\left(\mathbb{R}^{n}\right)$. Then

(i) $f \in H_{A}^{p(\cdot), q}\left(\mathbb{R}^{n}\right)$ if and only if $f \in \mathcal{S}_{0}^{\prime}\left(\mathbb{R}^{n}\right)$ and $S(f) \in L^{p(\cdot), q}\left(\mathbb{R}^{n}\right)$. Moreover, there exists a positive constant $C$ such that, for any $f \in H_{A}^{p(\cdot), q}\left(\mathbb{R}^{n}\right)$,

$$
\frac{1}{C}\|S(f)\|_{L^{p(\cdot), q\left(\mathbb{R}^{n}\right)}} \leq\|f\|_{H_{A}^{p(\cdot), q}\left(\mathbb{R}^{n}\right)} \leq C\|S(f)\|_{L^{p(\cdot), q\left(\mathbb{R}^{n}\right)}} .
$$

(ii) The conclusion as in (i) remains true if $S(f)$ is replaced, respectively, by $g(f)$ or $g_{\lambda}^{*}(f)$ with $\lambda \in\left(1+\frac{2}{\min \left\{p_{-,}, 2\right\}}, \infty\right)$.

Remark 5.2. We point out that, in the original proof of the sufficiency of Theorem 5.4(i) (namely, [78, Theorem 5.2]), the method used therein is the same as that used in the proof of the sufficiency of [79, Theorem 2.7]; see [78, p. 385]. Thus,

(i) similarly to Remark 3.2, the following fact is needed: for any $f \in \mathcal{S}_{0}^{\prime}\left(\mathbb{R}^{n}\right)$, the $L^{p(\cdot), q}\left(\mathbb{R}^{n}\right)$ quasi-norms of the anisotropic Lusin area function $S(f)$ are independent of the choices of $\theta$ and $\psi$ as in Lemma 3.1. However, in the original proof of the sufficiency of [78, Theorem 5.2], the authors did not give the proof of this necessary fact. In the present article, we seal this gap in Theorem 5.3 above;

(ii) similarly to Remark 3.3, both the equalities (3.39) and (3.40) are also used in the original proof of the sufficiency of [78, Theorem 5.2]. However, the authors therein did not prove these two equalities. Indeed, by a proof similar to that of the sufficiency of Theorem 3.4(i) (or the sufficiency of Theorem 4.4(i)]), we can also seal these gaps existing in the original proof of the sufficiency of [78, Theorem 5.2]; we omit the details. 


\subsection{Real interpolation between $H_{A}^{p(\cdot)}\left(\mathbb{R}^{n}\right)$ and $L^{\infty}\left(\mathbb{R}^{n}\right)$}

For any given compatible couple of quasi-normed spaces $\left(X_{1}, X_{2}\right)$, their real interpolation space $\left(X_{1}, X_{2}\right)_{\theta, q}$, with $\theta \in(0,1)$ and $q \in(0, \infty]$, is defined as in (3.41).

In [78, Theorem 6.2], the authors obtained the following real interpolation result.

Theorem 5.5. Let $p(\cdot) \in C^{\log }\left(\mathbb{R}^{n}\right), q \in(0, \infty]$ and $\theta \in(0,1)$. Then it holds true that

$$
\left(H_{A}^{p(\cdot)}\left(\mathbb{R}^{n}\right), L^{\infty}\left(\mathbb{R}^{n}\right)\right)_{\theta, q}=H_{A}^{\widetilde{p}(\cdot), q}\left(\mathbb{R}^{n}\right),
$$

where $\frac{1}{\tilde{p}(\cdot)}=\frac{1-\theta}{p(\cdot)}$.

As a consequence of Theorem 5.5, [119, Corollary 4.20] and [59, Remark 4.2(ii)], we immediately obtain the following conclusion.

Corollary 5.1. Let $p(\cdot) \in C^{\log }\left(\mathbb{R}^{n}\right)$. If $p_{-} \in(1, \infty)$ and $q \in(0, \infty]$, then

$$
H_{A}^{p(\cdot), q}\left(\mathbb{R}^{n}\right)=L^{p(\cdot), q}\left(\mathbb{R}^{n}\right)
$$

with equivalent quasi-norms.

Remark 5.3. (i) When $p(\cdot) \equiv p \in(0,1]$, Theorem 5.5 goes back to [77, Lemma 6.3], which states that

$$
\left(H_{A}^{p}\left(\mathbb{R}^{n}\right), L^{\infty}\left(\mathbb{R}^{n}\right)\right)_{\theta, q}=H_{A}^{p /(1-\theta), q}\left(\mathbb{R}^{n}\right), \quad \theta \in(0,1) \quad \text { and } \quad q \in(0, \infty]
$$

(ii) When $p(\cdot) \equiv p \in(1, \infty)$, Theorem 5.5 coincides with [77, Remark 6.7] (see also [89, Theorem 7]), namely,

$$
\left(L^{p}\left(\mathbb{R}^{n}\right), L^{\infty}\left(\mathbb{R}^{n}\right)\right)_{\theta, q}=L^{p /(1-\theta), q}\left(\mathbb{R}^{n}\right), \quad \theta \in(0,1) \quad \text { and } \quad q \in(0, \infty] .
$$

(iii) Let $A:=d \mathrm{I}_{n \times n}$ for some $d \in \mathbb{R}$ with $|d| \in(1, \infty)$. Then $H_{A}^{p}\left(\mathbb{R}^{n}\right)$ and $H_{A}^{p /(1-\theta), q}\left(\mathbb{R}^{n}\right)$ in (i) of this remark become, respectively, the classical isotropic Hardy and HardyLorentz spaces. In this case, the result in (i) of this remark is just [37, Theorem 1]. In addition, $H_{A}^{p(\cdot)}\left(\mathbb{R}^{n}\right)$ and $H_{A}^{\widetilde{p}(\cdot), q}\left(\mathbb{R}^{n}\right)$ in Theorem 5.5 become, respectively, the classical isotropic variable Hardy and Hardy-Lorentz spaces. In this case, Theorem 5.5 includes the result in [120, Theorem 1.5] as a special case, and Theorem 5.5 with $p_{-} \in(1, \infty)$ coincides with [59, Remark 4.2(ii)]. 


\subsection{Some applications}

The following boundedness of the maximal $\theta$-operator from $H_{A}^{p(\cdot), q}\left(\mathbb{R}^{n}\right)$ to $L^{p(\cdot), q}\left(\mathbb{R}^{n}\right)$ is just [76, Theorem 2.17].

Theorem 5.6. Let $\theta$ and $\theta_{0}$ be, respectively, as in (4.27) and (4.28) satisfying that there exists some constant $\beta \in(1, \infty)$ such that, for any $\alpha \in \mathbb{Z}_{+}^{n}$ and $x \in \mathbb{R}^{n} \backslash\left\{\overrightarrow{0}_{n}\right\}$,

$$
\left|\partial^{\alpha} \widehat{\theta_{0}}(x)\right| \leq C_{(\alpha, \beta)}|x|^{-\beta},
$$

where the positive constant $C_{(\alpha, \beta)}$ is independent of $x$. If $p(\cdot) \in C^{\log }\left(\mathbb{R}^{n}\right), q \in(0, \infty]$,

$$
\beta \in\left(\frac{\ln b}{\ln \lambda_{-}}, \infty\right) \text { and } p_{-} \in\left(\frac{\ln b}{\beta \ln \lambda_{-}}, \infty\right)
$$

then there exists a positive constant $C_{\left(p_{-}, p_{+}, q\right)}$, with $p_{-}$and $p_{+}$as in (4.1), such that, for any $f \in H_{A}^{p(\cdot), q}\left(\mathbb{R}^{n}\right)$,

$$
\left\|\sigma_{*}^{\theta} f\right\|_{L^{p(\cdot), q\left(\mathbb{R}^{n}\right)}} \leq C_{\left(p_{-}, p_{+}, q\right)}\|f\|_{H_{A}^{p(\cdot), q}\left(\mathbb{R}^{n}\right)^{\prime}}
$$

where $\sigma_{*}^{\theta} f$ denotes the maximal $\theta$-operator of $f$ as in (4.29).

Remark 5.4. A comment similar to Remark 4.3 also holds true for Theorem 5.6.

Corollary 5.2. Let all the assumptions be the same as in Theorem 5.6.

(i) If $q \in(0, \infty)$ and $f \in H_{A}^{p(\cdot), q}\left(\mathbb{R}^{n}\right)$, then $\sigma_{m}^{\theta} f$ converges pointwisely almost everywhere as well as in the $L^{p(\cdot), q}\left(\mathbb{R}^{n}\right)$ quasi-norm as $m \rightarrow \infty$;

(ii) If $q \in(0, \infty)$ and $f \in H_{A}^{p(\cdot), q}\left(\mathbb{R}^{n}\right)$ satisfy that there exists a subset $I \subset \mathbb{R}^{n}$ such that the restriction $\left.f\right|_{I} \in L^{r(\cdot), s}(I)$ with $r_{-} \in[1, \infty)$ and $s \in[1, \infty]$, then

$$
\lim _{m \rightarrow \infty} \sigma_{m}^{\theta} f(x)=f(x)
$$

pointwisely for almost every $x \in I$ as well as in the $L^{p(\cdot), q}(I)$ quasi-norm;

(iii) If $p_{-} \in(1, \infty), q \in[1, \infty)$ and $f \in L^{p(\cdot), q}\left(\mathbb{R}^{n}\right)$, then

$$
\lim _{m \rightarrow \infty} \sigma_{m}^{\theta} f(x)=f(x)
$$

pointwisely for almost every $x \in \mathbb{R}^{n}$ as well as in the $L^{p(\cdot), q}\left(\mathbb{R}^{n}\right)$ norm.

As special cases of Theorem 5.6, we further obtained the following conclusions (see [76, Theorems 2.25 and 2.28]).

Theorem 5.7. Let $p(\cdot) \in C^{\log }\left(\mathbb{R}^{n}\right)$ and $q \in(0, \infty]$. 
(i) If $\theta_{0}$ is as in (4.30), with

$\alpha \in\left(\max \left\{\frac{n-1}{2}, \frac{\ln b}{\ln \lambda_{-}}-\frac{n+1}{2}\right\}, \infty\right)$ and $p_{-} \in\left(\frac{\ln b}{\ln \lambda_{-}(n / 2+\alpha+1 / 2)}, \infty\right)$,

then there exists a positive constant $C_{\left(p_{-}, p_{+}, q\right)}$, with $p_{-}$and $p_{+}$as in (4.1), such that, for any $f \in H_{A}^{p(\cdot), q}\left(\mathbb{R}^{n}\right)$,

$$
\left\|\sigma_{*}^{\theta} f\right\|_{L^{p(\cdot), q\left(\mathbb{R}^{n}\right)}} \leq C_{\left(p_{-,} p_{+}, q\right)}\|f\|_{H_{A}^{p(\cdot), q}\left(\mathbb{R}^{n}\right)} .
$$

(ii) If $\theta_{0}$ is as in (4.31), then (5.1) also holds true for any $f \in H_{A}^{p(\cdot), q}\left(\mathbb{R}^{n}\right)$ with $p_{-} \in(0, \infty)$.

Remark 5.5. (i) Let $\theta_{0}$ be as in (4.30) or (4.31). Then the corresponding conclusions in Corollary 5.2 hold true as well.

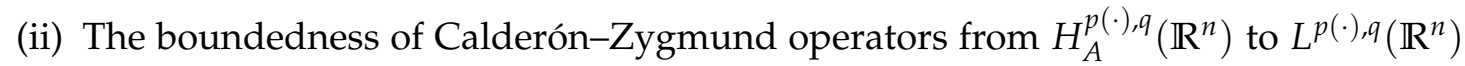
(or to itself) was obtained by Liu et al. in [82].

(iii) We point out that the dual spaces and the characterizations, respectively, in terms of wavelets and molecules, of the anisotropic variable Hardy-Lorentz space $H_{A}^{p(\cdot), q}\left(\mathbb{R}^{n}\right)$ are still unknown.

(iv) A remark similar to Remark 4.5(iii) should be also made for the space $H_{A}^{p(\cdot), q}\left(\mathbb{R}^{n}\right)$.

(v) Moreover, observe that the exponent $q \in(0, \infty]$ in $H_{A}^{p(\cdot), q}\left(\mathbb{R}^{n}\right)$ is only a constant. If the exponent $q$ is replaced by a variable exponent function,

$$
q(\cdot): \mathbb{R}^{n} \rightarrow(0, \infty]
$$

satisfying some reasonable conditions (for instance, the so-called globally logHölder continuity condition), then an interesting question is how to develop a real-variable theory of this sort of anisotropic variable Hardy-Lorentz spaces $H_{A}^{p(\cdot), q(\cdot)}\left(\mathbb{R}^{n}\right)$.

\section{Anisotropic Musielak-Orlicz Hardy spaces}

In this section, we first recall the notion of anisotropic Musielak-Orlicz Hardy spaces $H_{A}^{\varphi}\left(\mathbb{R}^{n}\right)$ introduced in [68] and then give their dual spaces as well as characterizations via molecules and wavelets, established recently in $[73,74]$. 


\subsection{Definition of anisotropic Musielak-Orlicz Hardy spaces}

We begin with recalling the classes of uniform anisotropic Muckenhoupt weights associated with $A$ from [68] as follows.

Definition 6.1. Let $q \in[1, \infty)$. The class of uniform anisotropic Muckenhoupt weights, $\mathcal{A}_{q}(A):=\mathcal{A}_{q}\left(\mathbb{R}^{n} ; A\right)$, is defined to be the set of all measurable functions $\varphi: \mathbb{R}^{n} \times$ $[0, \infty) \rightarrow[0, \infty)$ satisfying, when $q \in(1, \infty)$,

$$
\sup _{t \in(0, \infty)} \sup _{B \in \mathfrak{B}}\left\{\frac{1}{|B|} \int_{B} \varphi(y, t) d y\right\}\left\{\frac{1}{|B|} \int_{B}[\varphi(y, t)]^{-\frac{1}{q-1}} d y\right\}^{q-1}<\infty
$$

and, when $q=1$,

$$
\sup _{t \in(0, \infty)} \sup _{B \in \mathfrak{B}}\left\{\frac{1}{|B|} \int_{B} \varphi(y, t) d y\right\}\left\{\underset{y \in B}{\operatorname{ess} \sup }[\varphi(y, t)]^{-1}\right\}<\infty,
$$

where $\mathfrak{B}$ is as in (2.1). Moreover, let

$$
\mathcal{A}_{\infty}(A):=\bigcup_{q \in[1, \infty)} \mathcal{A}_{q}(A) .
$$

Recall also that a function $\Phi:[0, \infty) \rightarrow[0, \infty)$ is called an Orlicz function if $\Phi$ is non-decreasing, $\Phi(0)=0, \lim _{t \rightarrow \infty} \Phi(t)=\infty$ and, for any $t \in(0, \infty), \Phi(t) \in(0, \infty)$ (see, for instance, [60]). For a given function $\varphi: \mathbb{R}^{n} \times[0, \infty) \rightarrow[0, \infty)$ satisfying, for any given $x \in \mathbb{R}^{n}, \varphi(x, \cdot)$ is an Orlicz function, $\varphi$ is said to be of uniformly upper (resp. lower) type $p$ for some $p \in(-\infty, \infty)$ if there exists a positive constant $C$ such that, for almost every $x \in \mathbb{R}^{n}, s \in[1, \infty)$ (resp. $\left.s \in(0,1)\right)$ and $t \in[0, \infty), \varphi(x, s t) \leq C_{s}^{p} \varphi(x, t)$. Denote by $i(\varphi)$ the critical uniformly lower type index of $\varphi$, namely,

$$
i(\varphi):=\sup \{p \in(-\infty, \infty): \varphi \text { is of uniformly lower type } p\} .
$$

The following notion of anisotropic growth functions is just [68, Definition 3].

Definition 6.2. A function $\varphi: \mathbb{R}^{n} \times[0, \infty) \rightarrow[0, \infty)$ is called an anisotropic growth function if $\varphi$ satisfies the following conditions:

(i) $\varphi$ is a Musielak-Orlicz function, namely,

(i) $)_{1}$ for each given $x \in \mathbb{R}^{n}, \varphi(x, \cdot):[0, \infty) \rightarrow[0, \infty)$ is an Orlicz function;

(i) $)_{2}$ for each given $t \in[0, \infty), \varphi(\cdot, t)$ is a Lebesgue measurable function on $\mathbb{R}^{n}$.

(ii) $\varphi \in \mathcal{A}_{\infty}(A)$.

(iii) $\varphi$ is of uniformly lower type $p$ for some $p \in(0,1]$ and of uniformly upper type 1 . 
For any $\varphi \in \mathcal{A}_{\infty}(A)$, let

$$
q(\varphi):=\inf \left\{q \in[1, \infty): \varphi \in \mathcal{A}_{q}(A)\right\} .
$$

For any $\varphi$ as in Definition 6.2(i), the Musielak-Orlicz space $L^{\varphi}\left(\mathbb{R}^{n}\right)$ is defined to be the set of all measurable functions $f$ with their quasi-norms

$$
\|f\|_{L^{\varphi}\left(\mathbb{R}^{n}\right)}:=\inf \left\{\lambda \in(0, \infty): \int_{\mathbb{R}^{n}} \varphi(x,|f(x)| / \lambda) d x \leq 1\right\}<\infty .
$$

Moreover, in [68, Definition 5], the authors introduced the anisotropic MusielakOrlicz Hardy spaces as follows.

Definition 6.3. Let $N \in \mathbb{N}$ and $\varphi$ be an anisotropic growth function as in Definition 6.2. The anisotropic Musielak-Orlicz Hardy space $H_{N, A}^{\varphi}\left(\mathbb{R}^{n}\right)$ is defined by setting

$$
H_{N, A}^{\varphi}\left(\mathbb{R}^{n}\right):=\left\{f \in \mathcal{S}^{\prime}\left(\mathbb{R}^{n}\right): M_{N}(f) \in L^{\varphi}\left(\mathbb{R}^{n}\right)\right\}
$$

and, for any $f \in H_{N, A}^{\varphi}\left(\mathbb{R}^{n}\right)$, let $\|f\|_{H_{N, A}^{\varphi}\left(\mathbb{R}^{n}\right)}:=\left\|M_{N}(f)\right\|_{L^{\varphi}\left(\mathbb{R}^{n}\right)}$, where $M_{N}(f)$ denotes the non-tangential grand maximal function of $f \in \mathcal{S}^{\prime}\left(\mathbb{R}^{n}\right)$ as in (3.4).

Remark 6.1. (i) By [68, Theorem 33], we know that the space $H_{N, A}^{\varphi}\left(\mathbb{R}^{n}\right)$ is independent of the choice of $N$ as long as $N \in \mathbb{N} \cap[m(\varphi), \infty)$, here and thereafter, for any given $\varphi$ as in Definition 6.2,

$$
m(\varphi):=\left\lfloor\left[\frac{q(\varphi)}{i(\varphi)}-1\right] \frac{\ln b}{\ln \lambda_{-}}\right\rfloor
$$

with $q(\varphi)$ and $i(\varphi)$, respectively, as in (6.2) and (6.1). Thus, throughout this article, we always denote simply by $H_{A}^{\varphi}\left(\mathbb{R}^{n}\right)$ the anisotropic Musielak-Orlicz Hardy space.

(ii) Recall that, in Definition 4.1 (see also [75, Definition 2.4]), we present the anisotropic variable Hardy space $H_{A}^{p(\cdot)}\left(\mathbb{R}^{n}\right)$ with $p(\cdot) \in C^{\log }\left(\mathbb{R}^{n}\right)$ (see (4.2) and (4.3)). Similarly to [114, Remark 2.8], we know that, if

$$
\varphi(x, t):=t^{p(x)} \quad \text { for any } x \in \mathbb{R}^{n} \text { and } t \in(0, \infty),
$$

then $H_{A}^{\varphi}\left(\mathbb{R}^{n}\right)=H_{A}^{p(\cdot)}\left(\mathbb{R}^{n}\right)$. However, a general Musielak-Orlicz growth function $\varphi$ as in Definition 6.2 may not have the form as in (6.4). On another hand, as was proved in [114, Remark 2.14(iii)], there exists a variable exponent function $p(\cdot)$ satisfying (4.2) and (4.3) which were required in Definition 4.1 , but $t^{p(\cdot)}$ is not a uniform Muckenhoupt weight which was required in Definitions 6.2 and 6.3. Thus, the anisotropic Musielak-Orlicz Hardy space $H_{A}^{\varphi}\left(\mathbb{R}^{n}\right)$ and the anisotropic variable Hardy space $H_{A}^{p(\cdot)}\left(\mathbb{R}^{n}\right)$ cannot cover each other. 
(iii) When $A:=d \mathrm{I}_{n \times n}$ for some $d \in \mathbb{R}$ with $|d| \in(1, \infty)$, the space $H_{A}^{\varphi}\left(\mathbb{R}^{n}\right)$ becomes the Musielak-Orlicz Hardy space $H^{\varphi}\left(\mathbb{R}^{n}\right)$ (see [60]), which includes the classical Hardy space of Fefferman and Stein [38] and the classical weighted Hardy space of García-Cuerva [43] as well as the classical Orlicz-Hardy space of Janson [54] as special cases. In addition, if, for any $p \in(0,1], x \in \mathbb{R}^{n}$ and $t \in(0, \infty)$,

$$
\varphi(x, t):=w(x) t^{p}
$$

with $w \in \mathbb{A}_{\infty}(A)$ being an anisotropic $\mathbb{A}_{\infty}$ Muckenhoupt weight (see, for instance, [16]), then the space $H_{A}^{\varphi}\left(\mathbb{R}^{n}\right)$ coincides with the weighted anisotropic Hardy space $H_{w}^{p}\left(\mathbb{R}^{n} ; A\right)$ (see [16]), which includes the anisotropic Hardy space $H_{A}^{p}\left(\mathbb{R}^{n}\right)$ of Bownik [12] as a special case.

(iv) We should point out that Li et al. [68] also characterized $H_{A}^{\varphi}\left(\mathbb{R}^{n}\right)$, respectively, in terms of the radial or the non-tangential maximal function and the atom; see, respectively, [68, Theorems 9 and 40] for more details.

\subsection{Molecular characterizations of $H_{A}^{\varphi}\left(\mathbb{R}^{n}\right)$ and their applications}

Let $\varphi$ be as in Definition 6.2. For any measurable subset $E \subset \mathbb{R}^{n}$, the space $L_{\varphi}^{q}(E)$ with $q \in[1, \infty]$ is defined to be the set of all measurable functions $f$ on $E$ satisfying

$$
\|f\|_{L_{\varphi}^{q}(E)}:= \begin{cases}\sup _{t \in(0, \infty)}\left[\frac{1}{\varphi(E, t)} \int_{E}|f(x)|^{q} \varphi(x, t) d x\right]^{1 / q}<\infty, & \text { when } q \in[1, \infty), \\ \|f\|_{L^{\infty}(E)}<\infty, & \text { when } q=\infty,\end{cases}
$$

here and thereafter, for any $t \in[0, \infty), \varphi(E, t):=\int_{E} \varphi(x, t) d x$ (see [60]).

The following notion of anisotropic Musielak-Orlicz molecules is just [71, Definition 2.8], which is an anisotropic version of [50, Definition 4.4].

Definition 6.4. Let $\varphi$ be as in Definition 6.2, $q \in(q(\varphi), \infty]$ with $q(\varphi)$ as in (6.2), $s \in$ $\mathbb{Z}_{+}$and $\varepsilon \in(0, \infty)$. A measurable function $m$ is called an anisotropic Musielak-Orlicz $(\varphi, q, s, \varepsilon)$-molecule (shortly, a $(\varphi, q, s, \varepsilon)$-molecule), associated to some dilated ball $B:=$ $x_{0}+B_{i_{0}} \in \mathfrak{B}$ with $x_{0} \in \mathbb{R}^{n}, i_{0} \in \mathbb{Z}$ and $\mathfrak{B}$ as in (2.1), if

(i) for each $j \in \mathbb{Z}_{+}$,

$$
\|m\|_{L_{\varphi}^{q}\left(U_{j}(B)\right)} \leq b^{-j \varepsilon}\left\|\mathbf{1}_{B}\right\|_{L^{\varphi}\left(\mathbb{R}^{n}\right)^{\prime}}^{-1}
$$

where $U_{0}(B):=B$ and, for any $j \in \mathbb{N}$,

$$
U_{j}(B)=U_{j}\left(x_{0}+B_{i_{0}}\right):=x_{0}+\left[\left(A^{j} B_{i_{0}}\right) \backslash\left(A^{j-1} B_{i_{0}}\right)\right] ;
$$

(ii) for any multi-index $\alpha \in \mathbb{Z}_{+}^{n}$ with $|\alpha| \leq s, \int_{\mathbb{R}^{n}} m(x) x^{\alpha} d x=0$. 
By using $(\varphi, q, s, \varepsilon)$-molecules, in [73, Definition 3.3], the authors originally introduced the anisotropic Musielak-Orlicz molecular Hardy space $H_{A, \text { mol }}^{\varphi, q, s}\left(\mathbb{R}^{n}\right)$ as follows.

Definition 6.5. Let $\varphi, q, s$ and $\varepsilon$ be as in Definition 6.4. The anisotropic Musielak-Orlicz molecular Hardy space $H_{A, \text { mol }}^{\varphi, q, \mathcal{\varepsilon}}\left(\mathbb{R}^{n}\right)$ is defined to be the set of all $f \in \mathcal{S}^{\prime}\left(\mathbb{R}^{n}\right)$ satisfying that there exist $\left\{\lambda_{i}\right\}_{i \in \mathbb{N}} \subset \mathbb{C}$ and a sequence of $(\varphi, q, s, \varepsilon)$-molecules, $\left\{m_{i}\right\}_{i \in \mathbb{N}}$, associated, respectively, to $\left\{B^{(i)}\right\}_{i \in \mathbb{N}} \subset \mathfrak{B}$ such that $f=\sum_{i \in \mathbb{N}} \lambda_{i} m_{i}$ in $\mathcal{S}^{\prime}\left(\mathbb{R}^{n}\right)$ and

$$
\sum_{i \in \mathbb{N}} \varphi\left(B^{(i)},\left|\lambda_{i}\right|\left\|m_{i}\right\|_{L_{\varphi}^{q}\left(B^{(i)}\right)}\right)<\infty .
$$

Moreover, for any $f \in H_{A, \text { mol }}^{\varphi, q, s}\left(\mathbb{R}^{n}\right)$, let

$$
\|f\|_{H_{A, \text { mol }}^{\varphi, q, s, \varepsilon}\left(\mathbb{R}^{n}\right)}:=\inf \left\{\Lambda\left(\left\{\lambda_{i} m_{i}\right\}_{i \in \mathbb{N}}\right)\right\}
$$

with the infimum being taken over all the decompositions of $f$ as above, where

$$
\Lambda\left(\left\{\lambda_{i} m_{i}\right\}_{i \in \mathbb{N}}\right):=\inf \left\{\lambda \in(0, \infty): \sum_{i \in \mathbb{N}} \varphi\left(B^{(i)}, \frac{\left|\lambda_{i}\right|}{\lambda\left\|\mathbf{1}_{B^{(i)}}\right\|_{L^{\varphi}\left(\mathbb{R}^{n}\right)}}\right) \leq 1\right\} .
$$

The following molecular characterizations of $H_{A}^{\varphi}\left(\mathbb{R}^{n}\right)$ were established in [73, Theorem 3.12].

Theorem 6.1. Let $\varphi$ and $q$ be as in Definition 6.2, $s \in \mathbb{Z}_{+} \cap[m(\varphi), \infty)$ with $m(\varphi)$ as in (6.3), $N \in \mathbb{N} \cap[m(\varphi)+2, \infty)$ and

$$
\varepsilon \in\left(\max \left\{1,(s+1) \log _{b}\left(\lambda_{+} / \lambda_{-}\right)\right\}, \infty\right) .
$$

Then $H_{A}^{\varphi}\left(\mathbb{R}^{n}\right)=H_{A, \text { mol }}^{\varphi, q, s, \varepsilon}\left(\mathbb{R}^{n}\right)$ with equivalent quasi-norms.

Remark 6.2. (i) When $A$ is as in Remark 3.4(i), $H_{A}^{\varphi}\left(\mathbb{R}^{n}\right)$ and $H_{A, \text { mol }}^{\varphi, q, s, \varepsilon}\left(\mathbb{R}^{n}\right)$ become, respectively, the classical isotropic Musielak-Orlicz Hardy space (see [60]) and Musielak-Orlicz molecular Hardy space (see [50]. In this case, Theorem 6.1 coincides with [50, Theorem 4.13].

(ii) When $A$ is a diagonal expansive matrix (see [71]), Theorem 6.1 goes back to [71, Theorem 2.11]. In addition, $\mathrm{Li}$ et al. recently also obtained a kind of molecular characterizations for the space $H_{A}^{\varphi}\left(\mathbb{R}^{n}\right)$ (see [66, Theorem 2.10]), where the size condition of the $(\varphi, q, s, \varepsilon)$-molecule $m$ associated to some dilated ball $B$, namely,

$$
\|m\|_{L_{\varphi}^{q}(B)} \leq\left\|\mathbf{1}_{B}\right\|_{L^{\varphi}\left(\mathbb{R}^{n}\right)}^{-1}
$$

and, for any $j \in \mathbb{N}$ and $x \in U_{j}(B)$ with $U_{j}(B)$ as in Definition 6.2,

$$
|m(x)| \leq b^{-j \varepsilon}\left\|\mathbf{1}_{B}\right\|_{L^{\varphi}\left(\mathbb{R}^{n}\right)^{\prime}}^{-1}
$$

is much stronger than that used in Definition 6.4(i). In this sense, Theorem 6.1 extends the corresponding result obtained in [66, Theorem 2.10]. 
(iii) We should also point out that both [71, Theorem 2.11] and [66, Theorem 2.10] require the index $\varepsilon$ to belong to

$$
\left(\max \left\{q(\varphi) / i(\varphi), s \log _{b}\left(\lambda_{+}\right)+1\right\}, \infty\right)
$$

with $q(\varphi)$ and $i(\varphi)$, respectively, as in (6.2) and (6.1), which is just a proper subset of

$$
\left(\max \left\{1,(s+1) \log _{b}\left(\lambda_{+} / \lambda_{-}\right)\right\}, \infty\right)
$$

from (6.6). In this sense, the conclusion obtained in Theorem 6.1 is also stronger than that obtained in [71, Theorem 2.11] or [66, Theorem 2.10].

We next give some applications of Theorem 6.1 to the boundedness of linear operators. For this purpose, we need to recall the definition of anisotropic Musielak-Orlicz $(\varphi, q, s)$-atoms from [68, Definition 30].

Definition 6.6. Let $\varphi$ be as in Definition 6.2 and $q(\varphi)$ as in (6.2).

(i) An anisotropic triplet $(\varphi, q, s)$ is said to be admissible if $q \in(q(\varphi), \infty]$ and $s \in$ $\mathbb{Z}_{+} \cap[m(\varphi), \infty)$ with $m(\varphi)$ as in (6.3).

(ii) For any given anisotropic admissible triplet $(\varphi, q, s)$, a measurable function $a$ on $\mathbb{R}^{n}$ is called an anisotropic Musielak-Orlicz $(\varphi, q, s)$-atom (shortly, a $(\varphi, q, s)$-atom) if

(ii) ${ }_{1} \operatorname{supp} a:=\left\{x \in \mathbb{R}^{n}: a(x) \neq 0\right\} \subset B$, where $B \in \mathfrak{B}$ and $\mathfrak{B}$ is as in (2.1);

(ii) $)_{2}\|a\|_{L_{\varphi}^{q}\left(\mathbb{R}^{n}\right)} \leq\left\|\mathbf{1}_{B}\right\|_{L^{\varphi}\left(\mathbb{R}^{n}\right)}^{-1}$;

(ii) $)_{3}$ for any $\gamma \in \mathbb{Z}_{+}^{n}$ with $|\gamma| \leq s, \int_{\mathbb{R}^{n}} a(x) x^{\gamma} d x=0$.

In [73, Theorem 4.5], the authors established a criterion on the boundedness of linear operators on $H_{A}^{\varphi}\left(\mathbb{R}^{n}\right)$ as follows.

Theorem 6.2. Assume that $T$ is a linear operator defined on the set of all measurable functions. Let $(\varphi, q, \widetilde{s})$ be an anisotropic admissible triplet (see Definition 6.6(i)). If there exist some $j_{0} \in$ $\mathbb{Z}$ and a positive constant $C$ such that, for any $(\varphi, q, \widetilde{s})$-atom $\widetilde{a}$ supported in some dilated ball $x_{0}+B_{i_{0}} \in \mathfrak{B}$ with $x_{0} \in \mathbb{R}^{n}, i_{0} \in \mathbb{Z}$ and $\mathfrak{B}$ as in $(2.1), \frac{1}{C} T(\widetilde{a})$ is $a(\varphi, q, s, \varepsilon)$-molecule associated to $x_{0}+B_{i_{0}+j_{0}}$, where $s$ and $\varepsilon$ are as in Theorem 6.1, then $T$ has a unique bounded linear extension on $H_{A}^{\varphi}\left(\mathbb{R}^{n}\right)$.

In what follows, for any $r \in \mathbb{N}$, denote by $C^{r}\left(\mathbb{R}^{n}\right)$ the set of all functions on $\mathbb{R}^{n}$ whose derivatives with order not greater than $r$ are continuous. Recall that Liao et al. [71] introduced the following notion of integral anisotropic Calderón-Zygmund operators.

Definition 6.7. Let $r \in \mathbb{N}, q \in(1, \infty)$ and $\varphi \in \mathcal{A}_{q}(A)$. An anisotropic CalderónZygmund operator $T$ (see Definition 3.8(ii)) is called an integral anisotropic CalderónZygmund operator of order $r$ if its kernel $\mathcal{K}$ is a $C^{r}\left(\mathbb{R}^{n}\right)$ function with respect to 
the second variable $y$ and there exists a positive constant $C$ such that, for any dilated ball $B:=x_{0}+B_{i_{0}} \in \mathfrak{B}$ with $x_{0} \in \mathbb{R}^{n}, i_{0} \in \mathbb{Z}$ and $\mathfrak{B}$ as in (2.1), $m \in \mathbb{N}$, $x \in x_{0}+\left(B_{i_{0}+m+\tau+1} \backslash B_{i_{0}+m+\tau}\right)$ and $\alpha \in \mathbb{Z}_{+}^{n}$ with $1 \leq|\alpha| \leq r$,

$$
\left\|\int_{0}^{1}(1-t)^{|\alpha|-1} \partial_{y}^{\alpha} \widetilde{\mathcal{K}}\left(x, t A^{-i_{0}-m} \cdot\right) d t\right\|_{L_{\widetilde{\varphi}}^{q^{\prime}(B)}} \leq C b^{-i_{0}-m},
$$

where $\widetilde{\varphi}:=\varphi^{1 /(1-q)}, L_{\widetilde{\varphi}}^{q^{\prime}}(B)$ is as in (6.5) with $\varphi, E$ and $q$ replaced, respectively, by $\widetilde{\varphi}, B$ and $q^{\prime}$ and, for any $x, y \in \mathbb{R}^{n}$ satisfying $x \neq A^{i_{0}+m} y, \widetilde{\mathcal{K}}(x, y):=\mathcal{K}\left(x, A^{i_{0}+m} y\right)$.

Remark 6.3. (i) For any $r \in \mathbb{N}$, the anisotropic Calderón-Zygmund operator of order $r$ in [66, Definition 4.3], which originates from [12, p. 61, Definition 9.2], is an integral anisotropic Calderón-Zygmund operator of order $r$ as in Definition 6.7; see [71, Remark 4.4(i)] or [73, Remark 4.8(i)] for more details.

(ii) By [71, Remark 4.4(ii)], we know that, for any $r \in \mathbb{N}$, the classical isotropic Calderón-Zygmund operator of order $r$ (see [96, p. 289]) is also an operator as in Definition 6.7 in the case when $A:=d \mathrm{I}_{n \times n}$ for some $d \in \mathbb{R}$ with $|d| \in(1, \infty)$.

The following vanishing moment condition is just [66, Definition 4.5], which originates from [12, p. 64, Definition 9.4].

Definition 6.8. Let $\varphi$ be as in Definition 6.2, $r \in \mathbb{N}$ and

$$
\frac{q(\varphi)}{i(\varphi)}-1<\frac{\left(\ln \lambda_{-}\right)^{2}}{\ln b \ln \lambda_{+}} r
$$

where $q(\varphi)$ and $i(\varphi)$ are, respectively, as in (6.2) and (6.1). An integral anisotropic Calderón-Zygmund operator $T$ of order $r$ is said to satisfy $T^{*}\left(x^{\alpha}\right)=0$ for any $\alpha \in \mathbb{Z}_{+}^{n}$ with $|\alpha| \leq m(\varphi)$, where $m(\varphi)$ is as in (6.3), if, for any $f \in L^{2}\left(\mathbb{R}^{n}\right)$ with compact support and satisfying that, for any $\gamma \in \mathbb{Z}_{+}^{n}$ with $|\gamma| \leq r, \int_{\mathbb{R}^{n}} f(x) x^{\gamma} d x=0$, it holds true that, for any $\alpha \in \mathbb{Z}_{+}^{n}$ with $|\alpha| \leq m(\varphi), \int_{\mathbb{R}^{n}} T(f)(x) x^{\alpha} d x=0$.

As a further application of the criterion established in Theorem 6.2, we have the following boundedness of integral anisotropic Calderón-Zygmund operators from $H_{A}^{\varphi}\left(\mathbb{R}^{n}\right)$ to itself (or to $L^{\varphi}\left(\mathbb{R}^{n}\right)$ ); see [73, Theorems 4.13 and 4.14].

Theorem 6.3. Let $\varphi, r, q(\varphi), i(\varphi)$ and $m(\varphi)$ be as in Definition 6.8. Assume that $T$ is an integral anisotropic Calderón-Zygmund operator of order $r$ and satisfies $T^{*}\left(x^{\alpha}\right)=0$ for any $\alpha \in \mathbb{Z}_{+}^{n}$ with $|\alpha| \leq m(\varphi)$. Then there exists a positive constant $C$ such that, for any $f \in H_{A}^{\varphi}\left(\mathbb{R}^{n}\right)$,

$$
\|T(f)\|_{H_{A}^{\varphi}\left(\mathbb{R}^{n}\right)} \leq C\|f\|_{H_{A}^{\varphi}\left(\mathbb{R}^{n}\right)} .
$$

Theorem 6.4. Let $\varphi$ be as in Definition 6.2 and $m(\varphi)$ as in (6.3). Assume that $T$ is an integral anisotropic Calderón-Zygmund operator of order $\widetilde{r}$ with $\widetilde{r} \in \mathbb{N} \cap[m(\varphi)+1, \infty)$. Then there exists a positive constant $C$ such that, for any $f \in H_{A}^{\varphi}\left(\mathbb{R}^{n}\right)$,

$$
\|T(f)\|_{L^{\varphi}\left(\mathbb{R}^{n}\right)} \leq C\|f\|_{H_{A}^{\varphi}\left(\mathbb{R}^{n}\right)} .
$$


Remark 6.4. (i) When $A$ is a diagonal expansive dilation (see [71, p. 3]), Theorems 6.3 and 6.4 coincide, respectively, with [71, Theorems 4.7 and 4.6$]$.

(ii) By Remark 6.3(i), we know that Theorems 6.3 and 6.4 extend the corresponding results obtained in [66, Theorems 4.8 and 4.7], respectively.

(iii) Let $r \in \mathbb{N}$ and $p \in(0,1]$ satisfy

$$
\frac{1}{p}-1 \leq \frac{\left(\ln \lambda_{-}\right)^{2}}{\ln b \ln \lambda_{+}} r
$$

If, for any $x \in \mathbb{R}^{n}$ and $t \in(0, \infty), \varphi(x, t):=t^{p}$, then $q(\varphi)=1, i(\varphi)=p$ and $H_{A}^{\varphi}\left(\mathbb{R}^{n}\right)$ and $L^{\varphi}\left(\mathbb{R}^{n}\right)$ become the anisotropic Hardy space $H_{A}^{p}\left(\mathbb{R}^{n}\right)$ of Bownik [12] and the Lebesgue space $L^{p}\left(\mathbb{R}^{n}\right)$, respectively. In this case, by Theorems 6.3 and 6.4 and Remark 6.3(i), we find that, for any $r \in \mathbb{N}$ and $p \in(0,1]$ as in (6.7), the anisotropic Calderón-Zygmund operator of order $r$ (see [12, p. 61, Definition 9.2]) is bounded from $H_{A}^{p}\left(\mathbb{R}^{n}\right)$ to itself (or to $L^{p}\left(\mathbb{R}^{n}\right)$ ) (see $[12$, p. 68, Theorem 9.8 and p. 69, Theorem 9.9]). Moreover, let $A:=d \mathrm{I}_{n \times n}$ for some $d \in \mathbb{R}$ with $|d| \in(1, \infty), r=1$. Then $\frac{\left(\ln \lambda_{-}\right)^{2}}{\ln b \ln \lambda_{+}} r=\frac{1}{n}$ and $H_{A}^{\varphi}\left(\mathbb{R}^{n}\right)$ and $L^{\varphi}\left(\mathbb{R}^{n}\right)$ become the classical isotropic Hardy space $H^{p}\left(\mathbb{R}^{n}\right)$ and the Lebesgue space $L^{p}\left(\mathbb{R}^{n}\right)$, respectively. In this case, by Theorems 6.3 and 6.4, and Remark 6.3(ii), we further conclude that, for any $p \in\left(\frac{n}{n+1}, 1\right]$, the classical Calderón-Zygmund operator is bounded from $H^{p}\left(\mathbb{R}^{n}\right)$ to itself (or to $L^{p}\left(\mathbb{R}^{n}\right)$ ), which is a well-known result (see, for instance, $[3,96]$ ).

(iv) Let $r:=1$ and $p \in\left(\ln b /\left[\ln b+\ln \lambda_{-}\right], 1\right]$. If, for any $x \in \mathbb{R}^{n}$ and $t \in[0, \infty)$, $\varphi(x, t):=w(x) t^{p}$, where $w$ is an anisotropic $\mathbb{A}_{1}$ Muckenhoupt weight (see [16]), then $q(\varphi)=1, i(\varphi)=p$ and $H_{A}^{\varphi}\left(\mathbb{R}^{n}\right)$ becomes the weighted anisotropic Hardy space $H_{w}^{p}\left(\mathbb{R}^{n} ; A\right)$ (see [16]). In this case, by Theorem 6.3, we know that, for any $p \in$ $\left(\ln b /\left[\ln b+\ln \lambda_{-}\right], 1\right]$, the anisotropic Calderón-Zygmund operator (see Definition 3.8(ii)) is bounded on $H_{w}^{p}\left(\mathbb{R}^{n} ; A\right)$, which is just [118, Theorem 3.2].

(v) Recently, Sun et al. [99] established the molecular characterization of the anisotropic weak Musielak-Orlicz Hardy space $W H_{A}^{\varphi}\left(\mathbb{R}^{n}\right)$; as applications, in [99], they also obtained the boundedness of Calderón-Zygmund operators from $H_{A}^{\varphi}\left(\mathbb{R}^{n}\right)$ to $W H_{A}^{\varphi}\left(\mathbb{R}^{n}\right)$ in the critical case.

\subsection{Littlewood-Paley function characterizations of $H_{A}^{\varphi}\left(\mathbb{R}^{n}\right)$}

In this subsection, we present the characterizations of $H_{A}^{\varphi}\left(\mathbb{R}^{n}\right)$ in terms of the anisotropic Lusin area function, the anisotropic Littlewood-Paley $g$-function or $g_{\lambda}^{*}$-function; see [67]. Meanwhile, we also correct some errors existing in the proof of the sufficiency of [67, Theorem 2.8], namely, the Lusin area function characterizations of $H_{A}^{\varphi}\left(\mathbb{R}^{n}\right)$. 
Let $\varphi$ be a Musielak-Orlicz function on $\mathbb{R}^{n}$ (see Definition 6.2(i)). For any $r \in(0, \infty)$, the space $L^{\varphi}\left(\mathbb{R}^{n}, \ell^{r}\right)$ is defined to be the set of all $\left\{f_{i}\right\}_{i \in \mathbb{N}}$ of measurable functions on $\mathbb{R}^{n}$ satisfying

$$
\left(\sum_{i \in \mathbb{N}}\left|f_{j}\right|^{r}\right)^{1 / r} \in L^{\varphi}\left(\mathbb{R}^{n}\right)
$$

and, for any $\left\{f_{i}\right\}_{i \in \mathbb{N}} \in L^{\varphi}\left(\mathbb{R}^{n}, \ell^{r}\right)$, let

$$
\left\|\left\{f_{i}\right\}_{i \in \mathbb{N}}\right\|_{L^{\varphi}\left(\mathbb{R}^{n}, \ell^{r}\right)}:=\left\|\left(\sum_{i \in \mathbb{N}}\left|f_{i}\right|^{r}\right)^{1 / r}\right\|_{L^{\varphi}\left(\mathbb{R}^{n}\right)} .
$$

The following Fefferman-Stein vector-valued inequality on the Musielak-Orlicz space is just [67, Lemma 3.6].

Lemma 6.1. Let $r \in(1, \infty], \varphi$ be a Musielak-Orlicz function with uniformly lower type $p_{\varphi}^{-}$and uniformly upper type $p_{\varphi}^{+}, q \in(1, \infty)$ and $\varphi \in \mathcal{A}_{q}(A)$. If $q(\varphi)<p_{\varphi}^{-} \leq p_{\varphi}^{+}<\infty$ with $q(\varphi)$ as in (6.2), then there exists a positive constant $C$ such that, for any $\left\{f_{i}\right\}_{i \in \mathbb{N}} \in L^{\varphi}\left(\mathbb{R}^{n}, \ell^{r}\right)$,

$$
\int_{\mathbb{R}^{n}} \varphi\left(x,\left\{\sum_{i \in \mathbb{N}}\left[M_{\mathrm{HL}}\left(f_{i}\right)(x)\right]^{r}\right\}^{1 / r}\right) d x \leq C \int_{\mathbb{R}^{n}} \varphi\left(x,\left[\sum_{i \in \mathbb{Z}}\left|f_{i}(x)\right|^{r}\right]^{1 / r}\right) d x,
$$

where $M_{\mathrm{HL}}$ denotes the Hardy-Littlewood maximal operator as in (3.9).

Via Lemmas 6.1 and 3.4, similarly to Theorem 3.5, we obtain the following conclusion; the details are omitted.

Theorem 6.5. Let $\varphi$ be as in Definition 6.2 and $\theta, \psi$ as in Lemma 3.1 with $s \in \mathbb{Z}_{+} \cap[m(\varphi), \infty)$, where $m(\varphi)$ is as in (6.3). Then there exists a positive constant $C$ such that, for any $f \in \mathcal{S}_{0}^{\prime}\left(\mathbb{R}^{n}\right)$,

$$
\frac{1}{C}\left\|S_{\theta}(f)\right\|_{L^{\varphi}\left(\mathbb{R}^{n}\right)} \leq\left\|S_{\psi}(f)\right\|_{L^{\varphi}\left(\mathbb{R}^{n}\right)} \leq C\left\|S_{\theta}(f)\right\|_{L^{\varphi}\left(\mathbb{R}^{n}\right)} ，
$$

where $S_{\theta}(f)$ and $S_{\psi}(f)$ are the anisotropic Lusin area functions as in (3.8) defined, respectively, via $\theta$ and $\psi$.

Let $(\varphi, q, s)$ be an anisotropic admissible triplet as in Definition 6.6(i). Notice that a $(\varphi, q, s)$-atom as in Definition 6.6(ii) is also a $(\varphi, q, s, \varepsilon)$-molecule for any $\varepsilon \in(0, \infty)$ as in Definition 6.4. Thus, by [73, Lemma 3.7], we obtain the following conclusion.

Lemma 6.2. Let $(\varphi, q, s)$ be an anisotropic admissible triplet and $N \in \mathbb{N} \cap[m(\varphi)+2, \infty)$ with $m(\varphi)$ as in (6.3). Then there exists a positive constant $C_{(A, \varphi, q, s)}$, depending on $A, \varphi, q$ and $s$, such that, for any $\lambda \in(0, \infty)$ and $(\varphi, q, s)$-atom $\widetilde{a}$ supported in some dilated ball $B \in \mathfrak{B}$,

$$
\int_{\mathbb{R}^{n}} \varphi\left(x, \lambda M_{N}(\widetilde{a})(x)\right) d x \leq C_{(A, \varphi, q, s)} \varphi\left(B, \lambda\left\|\mathbf{1}_{B}\right\|_{L^{\varphi}\left(\mathbb{R}^{n}\right)}^{-1}\right)
$$

holds true, where $\mathfrak{B}$ is as in (2.1) and $M_{N}$ as in (3.4). 
In [67, Theorems 2.8, 3.1 and 3.9], Li et al. established the characterizations of $H_{A}^{\varphi}\left(\mathbb{R}^{n}\right)$, respectively, in terms of the anisotropic Lusin area function, the anisotropic LittlewoodPaley $g$-function or $g_{\lambda}^{*}$-function as follows.

Theorem 6.6. Let $q \in[1, \infty)$ and $\varphi \in \mathcal{A}_{q}(A)$ be as in Definition 6.2. Then

(i) $f \in H_{A}^{\varphi}\left(\mathbb{R}^{n}\right)$ if and only if $f \in \mathcal{S}_{0}^{\prime}\left(\mathbb{R}^{n}\right)$ and $S(f) \in L^{\varphi}\left(\mathbb{R}^{n}\right)$. Moreover, there exists a positive constant $C$ such that, for any $f \in H_{A}^{\varphi}\left(\mathbb{R}^{n}\right)$,

$$
\frac{1}{C}\|S(f)\|_{L^{\varphi}\left(\mathbb{R}^{n}\right)} \leq\|f\|_{H_{A}^{\varphi}\left(\mathbb{R}^{n}\right)} \leq C\|S(f)\|_{L^{\varphi}\left(\mathbb{R}^{n}\right)} .
$$

(ii) The conclusion as in (i) remains true if $S(f)$ is replaced, respectively, by $g(f)$ or $g_{\lambda}^{*}(f)$ with $\lambda \in(2 q / p, \infty)$.

To show the sufficiency of Theorem 6.6(i), we also need the following two lemmas, which are just, respectively, [71, Lemma 3.5] and [60, Lemma 4.3(i)].

Lemma 6.3. Let $\varphi$ be an anisotropic growth function as in Definition 6.2. Then there exists a positive constant $C$ such that, for any $\left\{\left(x, t_{i}\right)\right\}_{i \in \mathbb{N}} \subset \mathbb{R}^{n} \times[0, \infty)$,

$$
\varphi\left(x, \sum_{i \in \mathbb{N}} t_{i}\right) \leq C \sum_{i \in \mathbb{N}} \varphi\left(x, t_{i}\right) .
$$

Lemma 6.4. Let $\varphi$ be as in Lemma 6.3. Then, for any given positive constant $C$, there exists a positive constant $\widetilde{C}$ such that, for any $\lambda \in(0, \infty)$ and measurable function $f$ on $\mathbb{R}^{n}$,

$$
\int_{\mathbb{R}^{n}} \varphi\left(x, \frac{|f(x)|}{\lambda}\right) d x \leq C \text { implies }\|f\|_{L^{\varphi}\left(\mathbb{R}^{n}\right)} \leq \widetilde{C} \lambda .
$$

Now we prove the sufficiency of Theorem 6.6(i).

Proof of the sufficiency of Theorem 6.6(i). Let $\psi$ be as in Lemma 3.1, $f \in \mathcal{S}_{0}^{\prime}\left(\mathbb{R}^{n}\right)$ and $S(f) \in$ $L^{\varphi}\left(\mathbb{R}^{n}\right)$. Then, by Theorem 6.5, we know that $S_{\psi}(f) \in L^{\varphi}\left(\mathbb{R}^{n}\right)$. Therefore, to finish the proof of the sufficiency of Theorem 6.6(i), we need to prove that $f \in H_{A}^{\varphi}\left(\mathbb{R}^{n}\right)$ and

$$
\|f\|_{H_{A}^{\varphi}\left(\mathbb{R}^{n}\right)} \lesssim\left\|S_{\psi}(f)\right\|_{L^{\varphi}\left(\mathbb{R}^{n}\right)}
$$

To this end, for any $k \in \mathbb{Z}$, let $\Omega_{k}:=\left\{x \in \mathbb{R}^{n}: S_{\psi}(f)(x)>2^{k}\right\}$ and

$$
\mathfrak{Q}_{k}:=\left\{Q \in \mathfrak{Q}:\left|Q \cap \Omega_{k}\right|>\frac{|Q|}{2} \text { and }\left|Q \cap \Omega_{k+1}\right| \leq \frac{|Q|}{2}\right\} .
$$

Clearly, for any $Q \in \mathfrak{Q}$, there exists a unique $k \in \mathbb{Z}$ such that $Q \in \mathfrak{Q}_{k}$. Let $\left\{Q_{i}^{k}\right\}_{i}$ be the set of all maximal dyadic cubes in $\mathfrak{Q}_{k}$, namely, there exists no $Q \in \mathfrak{Q}_{k}$ such that $Q_{i}^{k} \varsubsetneqq Q$ for any $i$. 
For any $Q \in \mathfrak{Q}$, let

$$
\widehat{Q}:=\left\{(y, t) \in \mathbb{R}_{+}^{n+1}:=\mathbb{R}^{n} \times(0, \infty): y \in Q, t \sim b^{v \ell(Q)+u}\right\}
$$

where $t \sim b^{v \ell(Q)+u}$ means the same as in (3.18). Obviously, $\{\widehat{Q}\}_{Q \in \mathfrak{Q}}$ are mutually disjoint and

$$
\mathbb{R}_{+}^{n+1}=\bigcup_{k \in \mathbb{Z}} \bigcup_{i} B_{k, i}
$$

where, for any $k \in \mathbb{Z}$ and $i, B_{k, i}:=\bigcup_{Q \subset Q_{i}^{k}, Q \in \mathfrak{Q}_{k}} \widehat{Q}$. Then, by Lemma 3.3(ii), we easily find that $\left\{B_{k, i}\right\}_{k \in \mathbb{Z}, i}$ are also mutually disjoint.

Let $\psi$ and $\theta$ be as in Lemma 3.1 with $s \in \mathbb{Z}_{+} \cap[m(\varphi), \infty)$, where $m(\varphi)$ is as in (6.3). Then $\theta$ has the vanishing moments up to order $s$ and, for any $f \in \mathcal{S}_{0}^{\prime}\left(\mathbb{R}^{n}\right)$ such that $S_{\psi}(f) \in L^{\varphi}\left(\mathbb{R}^{n}\right)$, and for any $x \in \mathbb{R}^{n}$, similarly to (3.20), we have

$$
f(x)=\sum_{k \in \mathbb{Z}} f * \psi_{k} * \theta_{k}(x)=\int_{\mathbb{R}_{+}^{n+1}} f * \psi_{t}(y) * \theta_{t}(x-y) d y d m(t)
$$

in $\mathcal{S}^{\prime}\left(\mathbb{R}^{n}\right)$, where $m(t)$ denotes the counting measure on $\mathbb{R}$ as in (3.20). For each $k \in \mathbb{Z}, i$ and $x \in \mathbb{R}^{n}$, let

$$
h_{i}^{k}(x):=\int_{B_{k, i}} f * \psi_{t}(y) * \theta_{t}(x-y) d y d m(t) .
$$

Next we prove the sufficiency of Theorem 6.6(i) in three steps.

Step 1) The aim of this step is to show that

$$
\sum_{k \in \mathbb{Z}} \sum_{i} h_{i}^{k} \text { converges in } \mathcal{S}^{\prime}\left(\mathbb{R}^{n}\right)
$$

For this purpose, repeating the proofs of assertions (i) and (ii) in the proof of the sufficiency of Theorem 3.4(i) with some slight modifications, we conclude that, for any given $r \in(q(\varphi), \infty)$ with $q(\varphi)$ as in (6.2),

(i) for any $k \in \mathbb{Z}, i$ and $x \in \mathbb{R}^{n}$,

$$
h_{i}^{k}(x)=\sum_{Q \subset Q_{i}^{k}, Q \in \mathfrak{Q}_{k}} \int_{\widehat{Q}} f * \psi_{t}(y) \theta_{t}(x-y) d y d m(t)
$$

holds true in $\mathcal{S}^{\prime}\left(\mathbb{R}^{n}\right) ;$

(ii) for any $k \in \mathbb{Z}$ and $i, h_{i}^{k}=\lambda_{i}^{k} a_{i}^{k}$ is a multiple of a $(\varphi, r, s)$-atom, where, for any $k \in \mathbb{Z}$ and $i, \lambda_{i}^{k} \sim 2^{k}\left\|\mathbf{1}_{B_{i}^{k}}\right\|_{L^{\varphi}\left(\mathbb{R}^{n}\right)}$ with the positive equivalence constants independent of 
$k$ and $i$, and $a_{i}^{k}$ is a $(\varphi, r, s)$-atom satisfying, for any $r \in(q(\varphi), \infty), k \in \mathbb{Z}, i$ and $\gamma \in \mathbb{Z}_{+}^{n}$ as in Definition 6.6(ii) $)_{3}$

$$
\begin{aligned}
& \operatorname{supp} a_{i}^{k} \subset B_{i}^{k}:=x_{Q_{i}^{k}}+B_{v\left[\ell\left(Q_{i}^{k}\right)-1\right]+u+3 \tau} \text { with } v \text { and } u \text { as in Lemma 3.3(iv), } \\
& \left\|a_{i}^{k}\right\|_{L_{\varphi}^{q}\left(\mathbb{R}^{n}\right)} \leq\left\|\mathbf{1}_{B_{i}^{k}}\right\|_{L^{\varphi}\left(\mathbb{R}^{n}\right)}^{-1} \text { and } \int_{\mathbb{R}^{n}} a_{i}^{k}(x) x^{\gamma} d x=0 .
\end{aligned}
$$

To show (6.12), we next consider two cases: $i \in \mathbb{N}$ and $i \in\{1, \cdots, I\}$ with some $I \in \mathbb{N}$.

Case 1) $i \in \mathbb{N}$. In this case, by [68, Propositions 6 and 7], to prove (6.12), it suffices to show that

$$
\lim _{l \rightarrow \infty}\left\|\sum_{l \leq|k| \leq m} \sum_{l \leq i \leq m} \lambda_{i}^{k} a_{i}^{k}\right\|_{H_{A}^{\varphi}\left(\mathbb{R}^{n}\right)}=0
$$

Indeed, by the facts that $\lambda_{i}^{k} \sim 2^{k}\left\|\mathbf{1}_{B_{i}^{k}}\right\|_{L^{\varphi}\left(\mathbb{R}^{n}\right)},\left|Q_{i}^{k} \cap \Omega_{k}\right| \geq \frac{\left|Q_{i}^{k}\right|}{2}$, [73, Lemma 3.4] and the mutual disjointness of $\left\{Q_{i}^{k}\right\}_{i \in \mathbb{N}}$ for any fixed $k \in \mathbb{Z}$, we find that, for any $l, m \in \mathbb{N}$ and $\lambda \in(0, \infty)$,

$$
\begin{aligned}
& \sum_{l \leq|k| \leq m} \sum_{l \leq i \leq m} \varphi\left(B_{i}^{k}, \frac{\left|\lambda_{i}^{k}\right|}{\lambda\left\|\mathbf{1}_{B_{i}^{k}}\right\|_{L^{\varphi}\left(\mathbb{R}^{n}\right)}}\right) \\
\sim & \sum_{l \leq|k| \leq m} \sum_{l \leq i \leq m} \varphi\left(B_{i}^{k}, \frac{2^{k}}{\lambda}\right) \sim \sum_{l \leq|k| \leq m} \sum_{l \leq i \leq m} \varphi\left(Q_{i}^{k}, \frac{2^{k}}{\lambda}\right) \\
\sim & \sum_{l \leq|k| \leq m} \sum_{l \leq i \leq m} \varphi\left(Q_{i}^{k} \cap \Omega_{k}, \frac{2^{k}}{\lambda}\right) \lesssim \sum_{l \leq|k| \leq m} \varphi\left(\Omega_{k}, \frac{2^{k}}{\lambda}\right) .
\end{aligned}
$$

This, combined with [68, Theorem 40] and the fact that, for any $\lambda \in(0, \infty)$,

$$
\sum_{k \in \mathbb{Z}} \varphi\left(\Omega_{k}, \frac{2^{k}}{\lambda}\right) \lesssim \int_{\mathbb{R}^{n}} \varphi\left(x, \frac{S_{\psi}(f)(x)}{\lambda}\right) d x<\infty,
$$

further implies that (6.14) holds true and hence finishes the proof of (6.12) in Case 1).

Case 2) $i \in\{1, \cdots, I\}$ with some $I \in \mathbb{N}$. In this case, to show (6.12), it suffices to prove that

$$
\lim _{l \rightarrow \infty}\left\|\sum_{l \leq|k| \leq m} \sum_{i=1}^{I} \lambda_{i}^{k} a_{i}^{k}\right\|_{H_{A}^{\varphi}\left(\mathbb{R}^{n}\right)}=0 .
$$

Indeed, by a proof similar to that of (6.14), it is easy to see that (6.16) also holds true, which completes the proof of (6.12) in Case 2) and hence of (6.12). 
Step 2) In this step, we prove that

$$
f=\sum_{k \in \mathbb{Z}} \sum_{i} \lambda_{i}^{k} a_{i}^{k} \quad \text { in } \mathcal{S}^{\prime}\left(\mathbb{R}^{n}\right) .
$$

For this purpose, for any $x \in \mathbb{R}^{n}$, let

$$
\widetilde{f}(x):=\sum_{k \in \mathbb{Z}} \sum_{i} h_{i}^{k}(x)=\sum_{k \in \mathbb{Z}} \sum_{i} \int_{B_{k, i}} f * \psi_{t}(y) \theta_{t}(x-y) d y d m(t) \quad \text { in } \mathcal{S}^{\prime}\left(\mathbb{R}^{n}\right),
$$

where, for any $k \in \mathbb{Z}$ and $i, B_{k, i}$ is as in (6.10). Then, to show (6.17), it suffices to prove that

$$
f=\widetilde{f} \text { in } \mathcal{S}^{\prime}\left(\mathbb{R}^{n}\right)
$$

To do this, by the above assertion (i) and (6.9), we know that, for any given $r \in$ $(q(\varphi), \infty)$ and for any $k \in \mathbb{Z}, i$ and $x \in \mathbb{R}^{n}$,

$$
\begin{aligned}
h_{i}^{k}(x) & =\lim _{N \rightarrow \infty} \int_{0}^{\infty} \int_{\mathbb{R}^{n}} f * \psi_{t}(y) \theta_{t}(x-y) \mathbf{1}_{\substack{\cup \in Q_{i}^{k}, Q \in \mathfrak{Q}_{k} \\
|\ell(Q)| \leq N}} \hat{Q}(y, t) d y d m(t) \\
& =\lim _{N \rightarrow \infty} \int_{\gamma(N)}^{\eta(N)} \int_{\mathbb{R}^{n}} f * \psi_{t}(y) \theta_{t}(x-y) \mathbf{1}_{B_{k, i}}(y, t) d y d m(t)
\end{aligned}
$$

holds true in $\mathcal{S}^{\prime}\left(\mathbb{R}^{n}\right)$, where, for any $N \in \mathbb{N}, \gamma(N)$ and $\eta(N)$ are as in (3.32). For the convenience of symbols, we rewrite $\widetilde{f}$ as, for any $x \in \mathbb{R}^{n}$,

$$
\tilde{f}(x)=\sum_{\ell \in \mathbb{N}} \int_{R^{(\ell)}} f * \psi_{t}(y) \theta_{t}(x-y) d y d m(t),
$$

where $\left\{R^{(\ell)}\right\}_{\ell \in \mathbb{N}}$ is an arbitrary permutation of $\left\{B_{k, i}\right\}_{k \in \mathbb{Z}, i}$. For any $L \in \mathbb{N}$ and $x \in \mathbb{R}^{n}$, let

$$
\widetilde{f}_{L}(x):=f(x)-\sum_{\ell=1}^{L} \int_{R^{(\ell)}} f * \psi_{t}(y) \theta_{t}(x-y) d y d m(t) .
$$

Then, from (6.10), (6.11) and (6.19), it follows that, for any $L \in \mathbb{N}$ and $x \in \mathbb{R}^{n}$,

$$
\begin{aligned}
\tilde{f}_{L}(x)= & \lim _{N \rightarrow \infty} \int_{\gamma(N)}^{\eta(N)} \int_{\mathbb{R}^{n}} f * \psi_{t}(y) \theta_{t}(x-y) \mathbf{1}_{\cup_{\ell=1}^{\infty} R^{(\ell)}}(y, t) d y d m(t) \\
& \quad-\lim _{N \rightarrow \infty} \int_{\gamma(N)}^{\eta(N)} \int_{\mathbb{R}^{n}} f * \psi_{t}(y) \theta_{t}(x-y) \mathbf{1}_{\cup_{\ell=1}^{L} R^{(\ell)}}(y, t) d y d m(t) \\
= & \lim _{N \rightarrow \infty} \int_{\gamma(N)}^{\eta(N)} \int_{\mathbb{R}^{n}} f * \psi_{t}(y) \theta_{t}(x-y) \mathbf{1}_{\cup_{\ell=L+1}^{\infty} R^{(\ell)}}(y, t) d y d m(t)
\end{aligned}
$$


holds true in $\mathcal{S}^{\prime}\left(\mathbb{R}^{n}\right)$.

Note that $H_{A}^{\varphi}\left(\mathbb{R}^{n}\right)$ is continuously embedded into $\mathcal{S}^{\prime}\left(\mathbb{R}^{n}\right)$ (see [68, Proposition 6]). Therefore, to prove (6.18), we only need to show that

$$
\left\|\widetilde{f}_{L}\right\|_{H_{A}^{\varphi}\left(\mathbb{R}^{n}\right)} \rightarrow 0 \text { as } L \rightarrow \infty .
$$

To this end, we borrow some ideas from the proof of the atomic characterizations of $H_{A}^{\varphi}\left(\mathbb{R}^{n}\right)$ (see [68, Theorem 40]). Indeed, for any $\varepsilon \in(0,1), L \in \mathbb{N}$ and $x \in \mathbb{R}^{n}$, let

$$
\widetilde{f}_{L}^{(\varepsilon)}(x):=\int_{\varepsilon}^{\alpha / \varepsilon} \int_{\mathbb{R}^{n}} f * \psi_{t}(y) \theta_{t}(x-y) \mathbf{1}_{\cup_{\ell=L+1}^{\infty} R^{(\ell)}}(y, t) d y d m(t),
$$

where $\alpha:=b^{-v+2(u+1)}$ with $v$ and $u$ as in Lemma 3.3(iv). Then, by the Lebesgue dominated convergence theorem, we find that, for any given any $\varepsilon \in(0,1), L \in \mathbb{N}$ and $x \in \mathbb{R}^{n}$,

$$
\widetilde{f}_{L}^{(\varepsilon)}(x)=\sum_{\ell=L+1}^{\infty} \int_{\varepsilon}^{\alpha / \varepsilon} \int_{\mathbb{R}^{n}} f * \psi_{t}(y) \theta_{t}(x-y) \mathbf{1}_{R^{(\ell)}}(y, t) d y d m(t)=: \sum_{\ell=L+1}^{\infty} h_{\ell}^{(\varepsilon)}(x)
$$

in $\mathcal{S}^{\prime}\left(\mathbb{R}^{n}\right)$. Moreover, by some arguments similar to those used in the proofs of assertions (i) and (ii) in the proof of the sufficiency of Theorem 3.4(i) as well as in the proof of (6.14) with some slight modifications, we conclude that, for any $\varepsilon \in(0,1), r \in(q(\varphi), \infty)$, $L \in \mathbb{N}$ and $\ell \in \mathbb{N} \cap[L+1, \infty), h_{\ell}^{(\varepsilon)}$ is a multiple of a $(\varphi, r, s)$-atom, namely, there exist $\left\{\lambda_{\ell}\right\}_{\ell \in \mathbb{N} \cap[L+1, \infty)} \subset \mathbb{C}$ and a sequence of $(\varphi, r, s)$-atoms, $\left\{a_{\ell}^{(\varepsilon)}\right\}_{\ell \in \mathbb{N} \cap[L+1, \infty)}$, supported, respectively, in $\left\{B^{(\ell)}\right\}_{\ell \in \mathbb{N} \cap[L+1, \infty)} \subset \mathfrak{B}$ such that, for any $\ell \in \mathbb{N} \cap[L+1, \infty), h_{\ell}^{(\varepsilon)}=\lambda_{\ell} a_{\ell}^{(\varepsilon)}$, where, for any $\ell \in \mathbb{N} \cap[L+1, \infty), \lambda_{\ell}$ and $B^{(\ell)}$ are independent of $\varepsilon$. Therefore, for any $\varepsilon \in(0,1), L \in \mathbb{N}$ and $x \in \mathbb{R}^{n}$,

$$
\widetilde{f}_{L}^{(\varepsilon)}(x)=\sum_{\ell=L+1}^{\infty} \lambda_{\ell} a_{\ell}^{(\varepsilon)}(x) \quad \text { in } \mathcal{S}^{\prime}\left(\mathbb{R}^{n}\right)
$$

and

$$
\sum_{\ell=L+1}^{\infty} \varphi\left(B^{(\ell)}, \frac{\left|\lambda_{\ell}\right|}{\left\|\mathbf{1}_{B^{(\ell)}}\right\|_{L^{\varphi}\left(\mathbb{R}^{n}\right)}}\right)<\infty
$$

On another hand, for any given $\mathcal{N} \in \mathbb{N} \cap[m(\varphi)+2, \infty)$ with $m(\varphi)$ as in (6.3), let $M_{\mathcal{N}}$ denote the non-tangential grand maximal operator as in (3.4) with $N$ replaced by $\mathcal{N}$. Moreover, let $\varepsilon:=\gamma(N)$ with $N \in \mathbb{N} \cap\left[\left\lfloor\frac{-u-1}{v}\right\rfloor+1, \infty\right)$. Then, from (6.20), we deduce that

$$
M_{\mathcal{N}}\left(\widetilde{f}_{L}\right)=M_{\mathcal{N}}\left(\lim _{N \rightarrow \infty} \widetilde{f}_{L}^{(\gamma(N))}\right) \leq \liminf _{N \rightarrow \infty} M_{\mathcal{N}}\left(\widetilde{f}_{L}^{(\gamma(N))}\right)
$$


This, together with (6.22) and Lemmas 6.3 and 6.2, further implies that, for any $L \in \mathbb{N}$,

$$
\begin{aligned}
& \int_{\mathbb{R}^{n}} \varphi\left(x, M_{\mathcal{N}}\left(\tilde{f}_{L}\right)\right) d x \\
\lesssim & \int_{\mathbb{R}^{n}} \varphi\left(x, \liminf _{N \rightarrow \infty} M_{\mathcal{N}}\left(\widetilde{f}_{L}^{(\gamma(N))}\right)\right) d x \\
\lesssim & \int_{\mathbb{R}^{n}} \varphi\left(x, \liminf _{N \rightarrow \infty} \sum_{\ell=L+1}^{\infty}\left|\lambda_{\ell}\right| M_{\mathcal{N}}\left(a_{\ell}^{(\gamma(N))}\right)\right) d x \\
\lesssim & \sum_{\ell=L+1}^{\infty} \varphi\left(B^{(\ell)}, \frac{\left|\lambda_{\ell}\right|}{\left\|\mathbf{1}_{B^{(\ell)}}\right\|_{L^{\varphi}\left(\mathbb{R}^{n}\right)}}\right) .
\end{aligned}
$$

By this and (6.23), we easily know that (6.21) holds true, which completes the proof of (6.17).

Step 3) By (6.17), the facts that $\lambda_{i}^{k} \sim 2^{k}\left\|\mathbf{1}_{B_{i}^{k}}\right\|_{L^{\varphi}\left(\mathbb{R}^{n}\right)},\left|Q_{i}^{k} \cap \Omega_{k}\right| \geq \frac{\left|Q_{i}^{k}\right|}{2}$, [73, Lemma 3.4] and the mutual disjointness of $\left\{Q_{i}^{k}\right\}_{i \in \mathbb{N}}$ for any fixed $k \in \mathbb{Z}$, we find that, for any $\lambda \in(0, \infty)$,

$$
\begin{aligned}
& \sum_{k \in \mathbb{Z}} \sum_{i} \varphi\left(B_{i}^{k}, \frac{\left|\lambda_{i}^{k}\right|}{\lambda\left\|\mathbf{1}_{B_{i}^{k}}\right\|_{L^{\varphi}\left(\mathbb{R}^{n}\right)}}\right) \\
\sim & \sum_{k \in \mathbb{Z}} \sum_{i} \varphi\left(B_{i}^{k}, \frac{2^{k}}{\lambda}\right) \sim \sum_{k \in \mathbb{Z}} \sum_{i} \varphi\left(Q_{i}^{k}, \frac{2^{k}}{\lambda}\right) \\
\sim & \sum_{k \in \mathbb{Z}} \sum_{i} \varphi\left(Q_{i}^{k} \cap \Omega_{k}, \frac{2^{k}}{\lambda}\right) \lesssim \sum_{k \in \mathbb{Z}} \varphi\left(\Omega_{k}, \frac{2^{k}}{\lambda}\right) .
\end{aligned}
$$

From this and (6.15), it follows that, for any $\lambda \in(0, \infty)$,

$$
\sum_{k \in \mathbb{Z}} \sum_{i} \varphi\left(B_{i}^{k}, \frac{\left|\lambda_{i}^{k}\right|}{\lambda\left\|\mathbf{1}_{B_{i}^{k}}\right\|_{L^{\varphi}\left(\mathbb{R}^{n}\right)}}\right) \lesssim \int_{\mathbb{R}^{n}} \varphi\left(x, \frac{S_{\psi}(f)(x)}{\lambda}\right) d x,
$$

which, combined with [68, Theorem 40], implies that $f \in H_{A}^{\varphi}\left(\mathbb{R}^{n}\right)$ and (6.8) holds true. This finishes the proof of the sufficiency of Theorem 6.6(i).

Remark 6.5. (i) Let $S_{\theta}(f)$ and $S_{\psi}(f)$ be the anisotropic Lusin area functions defined, respectively, by using $\theta$ and $\psi$. We point out that, in the original proof of the sufficiency of Theorem 6.6(i) (namely, [67, Theorem 2.8]), the authors used both $S_{\theta}(f)$ and $S_{\psi}(f)$; see, respectively, the proofs of [67, (2.22) and (2.25)]. Thus, the following fact is needed: for any $f \in \mathcal{S}_{0}^{\prime}\left(\mathbb{R}^{n}\right)$, the $L^{\varphi}\left(\mathbb{R}^{n}\right)$ quasi-norms of the anisotropic Lusin area function $S(f)$ are independent of the choices of $\theta$ and $\psi$ as in Lemma 3.1. However, in the original proof of the sufficiency of [67, Theorem 2.8], the authors did not give out the proof of this necessary fact. To seal this gap, we present the above fact in Theorem 6.5. 
(ii) Let all symbols be the same as those used in the proof of the sufficiency of Theorem 6.6(i). Observe that, in the original proof of the sufficiency of Theorem 6.6(i) (namely, [67, Theorem 2.8]), the authors used the following two equalities: for any $f \in \mathcal{S}_{0}^{\prime}\left(\mathbb{R}^{n}\right)$ with $S(f) \in L^{\varphi}\left(\mathbb{R}^{n}\right)$, and $x \in \mathbb{R}^{n}$,

$$
\begin{aligned}
& \int_{\mathbb{R}_{+}^{n+1}} f * \psi_{t}(y) * \theta_{t}(x-y) d y d m(t) \\
= & \sum_{k \in \mathbb{Z}} \sum_{i} \int_{B_{k, i}} f * \psi_{t}(y) * \theta_{t}(x-y) d y d m(t)
\end{aligned}
$$

in $\mathcal{S}^{\prime}\left(\mathbb{R}^{n}\right)$ (see $\left[67\right.$, p. 294]) and, for each $k \in \mathbb{Z}, i$ and $x \in \mathbb{R}^{n}$,

$$
\begin{aligned}
& \int_{B_{k, i}} f * \psi_{t}(y) * \theta_{t}(x-y) d y d m(t) \\
= & \sum_{Q \subset Q_{i}^{k}, Q \in \mathfrak{Q}_{k}} \int_{\widehat{Q}} f * \psi_{t}(y) * \theta_{t}(x-y) d y d m(t)
\end{aligned}
$$

in $\mathcal{S}^{\prime}\left(\mathbb{R}^{n}\right)$ (see [67, p. 294 and (2.20)]); however, the authors therein did not prove these two equalities. In the present article, we give the proofs of both (6.24) and (6.25) and hence seal these gaps existing in the original proof of the sufficiency of [67, Theorem 2.8]; see, respectively, the proofs of (6.18) and (6.13) above for the details.

\subsection{Dual spaces with applications in wavelet characterizations of $H_{A}^{\varphi}\left(\mathbb{R}^{n}\right)$}

In this subsection, via establishing a John-Nirenberg inequality for elements from anisotropic Musielak-Orlicz Campanato spaces $\mathcal{L}_{A}^{\varphi, 1, s}\left(\mathbb{R}^{n}\right)$, we first present that the dual space of $H_{A}^{\varphi}\left(\mathbb{R}^{n}\right)$ is $\mathcal{L}_{A}^{\varphi, 1, s}\left(\mathbb{R}^{n}\right)$. Then, via this duality, we give the characterizations of $H_{A}^{\varphi}\left(\mathbb{R}^{n}\right)$ in terms of the so-called tight frame multiwavelets.

Let $A$ be some fixed dilation. For any $j \in \mathbb{Z}$ and $k \in \mathbb{Z}^{n}$, let $Q_{j, k}:=A^{-j}\left([0,1)^{n}+k\right)$ and

$$
\mathcal{Q}:=\bigcup_{j \in \mathbb{Z}} Q_{j}:=\bigcup_{j \in \mathbb{Z}}\left\{Q_{j, k}: k \in \mathbb{Z}^{n}\right\}
$$

For each $j \in \mathbb{Z}$ and $k \in \mathbb{Z}^{n}, Q_{j, k}$ is called a dilated cube (see, for instance, [15, p. 1475]). Clearly, for any $k_{1}, k_{2} \in \mathbb{Z}^{n}$ with $k_{1} \neq k_{2},\left|Q_{j, k_{1}} \cap Q_{j, k_{2}}\right|=0$. In addition, for each dilated cube $Q_{j, k}$, denote by $c_{Q_{j, k}}$ its center, by $x_{Q_{i, k}}$ its lower-left corner $A^{-j} k$ and by $\ell\left(Q_{j, k}\right)$ its level, namely, the integer $-j$. Then [14, Lemma 2.9(a)] implies that there exists some $j_{(A, n)}=: j_{0} \in \mathbb{N}$, only depending on $A$ and $n$, such that, for any $x \in Q_{j, k}$,

$$
B_{\rho}\left(c_{Q_{j, k}}, b^{-j_{0}-j}\right) \subset Q_{j, k} \subset B_{\rho}\left(x, b^{j_{0}-j}\right),
$$


where, for any $x \in \mathbb{R}^{n}$ and $r \in(0, \infty), B_{\rho}(x, r):=\left\{y \in \mathbb{R}^{n}: \rho(x-y)<r\right\}$.

For any $s \in \mathbb{Z}_{+}$, denote by $\mathcal{P}_{s}\left(\mathbb{R}^{n}\right)$ the set of all polynomials on $\mathbb{R}^{n}$ with degree not greater than $s$. For any given $f \in L_{\text {loc }}^{1}\left(\mathbb{R}^{n}\right)$ and for any $Q \in Q$ and $s \in \mathbb{Z}_{+}$, let $P_{Q}^{s} f$ be the unique polynomial $P \in \mathcal{P}_{S}\left(\mathbb{R}^{n}\right)$ such that, for any $R \in \mathcal{P}_{S}\left(\mathbb{R}^{n}\right)$,

$$
\int_{Q}[f(x)-P(x)] R(x) d x=0 .
$$

The following anisotropic Musielak-Orlicz Campanato space was first introduced in [74, Definition 3.1].

Definition 6.9. Let $\varphi$ be as in Definition 6.2, $q \in[1, \infty)$ and $s \in \mathbb{Z}_{+}$. The anisotropic Musielak-Orlicz Campanato space $\mathcal{L}_{A}^{\varphi, q, s}\left(\mathbb{R}^{n}\right)$ is defined to be the set of all $f \in L_{\text {loc }}^{1}\left(\mathbb{R}^{n}\right)$ such that their quasi-norms

$$
\begin{aligned}
\|f\|_{\mathcal{L}_{A}^{\varphi, q, s}\left(\mathbb{R}^{n}\right)} & :=\sup _{Q \in \mathcal{Q}} \frac{1}{\left\|\mathbf{1}_{Q}\right\|_{L^{\varphi}\left(\mathbb{R}^{n}\right)}}\left\{\int_{Q}\left[\frac{\left|f(x)-P_{Q}^{s} f(x)\right|}{\varphi\left(x,\left\|\mathbf{1}_{Q}\right\|_{L^{\varphi}\left(\mathbb{R}^{n}\right)}^{-1}\right)}\right]^{q} \varphi\left(x,\left\|\mathbf{1}_{Q}\right\|_{L^{\varphi}\left(\mathbb{R}^{n}\right)}^{-1}\right) d x\right\}^{1 / q} \\
& <\infty,
\end{aligned}
$$

where the supremum is taken over all the dilated cubes $Q \in Q$ with $Q$ as in (6.26).

Remark 6.6. (i) When $A:=d \mathrm{I}_{n \times n}$ for some $d \in \mathbb{R}$ with $|d| \in(1, \infty)$, the space $\mathcal{L}_{A}^{\varphi, q, s}\left(\mathbb{R}^{n}\right)$ becomes the Musielak-Orlicz Campanato space $\mathcal{L}_{\varphi, q, s}\left(\mathbb{R}^{n}\right)$ from [70]. Moreover,

(i) 1 if, for any given $p \in(0,1]$ and for any $x \in \mathbb{R}^{n}$ and $t \in(0, \infty), \varphi(x, t):=t^{p}$, then, $\mathcal{L}_{A}^{\varphi, q, S}\left(\mathbb{R}^{n}\right)$ is just the classical isotropic Campanato space $L_{1 / p-1, q, s}\left(\mathbb{R}^{n}\right)$ (see [20]);

(i) $)_{2}$ if, for any given $p \in(0,1]$ and for any $x \in \mathbb{R}^{n}$ and $t \in(0, \infty), \varphi(x, t):=w(x) t^{p}$ with $w$ being an $A_{\infty}$ Muckenhoupt weight (see, for instance, [43]), then, the space $\mathcal{L}_{A}^{\varphi, q, s}\left(\mathbb{R}^{n}\right)$ coincides with the weighted Campanato space; see [43] for $n=1$ and, see [117] for $n \in \mathbb{N}$.

(ii) When $q=1$ and $s=0$, the space $\mathcal{L}_{A}^{\varphi, q, s}\left(\mathbb{R}^{n}\right)$ becomes the anisotropic BMO space of Musielak-Orlicz type, $\mathrm{BMO}_{A}^{\varphi}\left(\mathbb{R}^{n}\right)$ (see [33]), which includes the classical space $\mathrm{BMO}\left(\mathbb{R}^{n}\right)$ of John and Nirenberg [56], the anisotropic BMO space $\mathrm{BMO}_{A}\left(\mathbb{R}^{n}\right)$ of Bownik [12], the weighted BMO space $\mathrm{BMO}_{w}\left(\mathbb{R}^{n}\right)$ of Muckenhoupt and Wheeden [86] and the Orlicz BMO-type space $\mathrm{BMO}_{\rho}\left(\mathbb{R}^{n}\right)$ of Janson [54] and Strömberg [98] as well as the Musielak-Orlicz BMO-type space $\mathrm{BMO}^{\varphi}\left(\mathbb{R}^{n}\right)$ of $\mathrm{Ky}[60]$ as special cases (see [33,70] for more details).

(iii) If, for any $p \in(0,1], x \in \mathbb{R}^{n}$ and $t \in(0, \infty), \varphi(x, t):=t^{p}$, then the space $\mathcal{L}_{A}^{\varphi, q, s}\left(\mathbb{R}^{n}\right)$ becomes the anisotropic Campanato space $C_{q, s}^{1 / p-1}\left(\mathbb{R}^{n}\right)$ of Bownik (see [12]). 
For any given $k \in \mathbb{Z}^{n}$ and for any $i \in \mathbb{N}$, we divide the cube $[0,1)^{n}+k$ into $2^{\text {in }}$ mutually disjoint subcubes of equal size and denote by $\Theta_{i}^{(k)}$ the set of these subcubes. Moreover, let

$$
\Theta(k):=\bigcup_{i \in \mathbb{N}} \Theta_{i}^{(k)} \text { and } \Theta:=\left\{A^{j} I: j \in \mathbb{Z} \text { and } I \in \Theta(k) \text { with } k \in \mathbb{Z}^{n}\right\} .
$$

In [74, Theorem 3.8], the authors established a John-Nirenberg inequality for functions from $\mathcal{L}_{A}^{\varphi, 1, s}\left(\mathbb{R}^{n}\right)$ as follows.

Theorem 6.7. Let $\varphi$ and $s$ be as in Definition 6.9. Then there exist positive constants $C_{1}, C_{2}$ and $C_{3}$ such that, for any $f \in \mathcal{L}_{A}^{\varphi, 1, s}\left(\mathbb{R}^{n}\right), Q \in Q \cup \Theta$ and $\beta \in(0, \infty)$, when $\varphi \in \mathcal{A}_{1}(A)$,

$$
\begin{aligned}
& \varphi\left(\left\{x \in Q: \frac{\left|f(x)-P_{Q}^{s} f(x)\right|}{\varphi\left(x,\left\|\mathbf{1}_{Q}\right\|_{L^{\varphi}\left(\mathbb{R}^{n}\right)}^{-1}\right)}>\beta\right\},\left\|\mathbf{1}_{Q}\right\|_{L^{\varphi}\left(\mathbb{R}^{n}\right)}^{-1}\right) \\
\leq & C_{1} \exp \left\{-\frac{C_{2} \beta}{\|f\|_{\mathcal{L}_{A}^{\varphi, 1, s}\left(\mathbb{R}^{n}\right)}\left\|\mathbf{1}_{Q}\right\|_{L^{\varphi}\left(\mathbb{R}^{n}\right)}}\right\}
\end{aligned}
$$

and, when $\varphi \in \mathcal{A}_{q}(A)$ for some $q \in(1, \infty)$,

$$
\begin{aligned}
& \varphi\left(\left\{x \in Q: \frac{\left|f(x)-P_{Q}^{s} f(x)\right|}{\varphi\left(x,\left\|\mathbf{1}_{Q}\right\|_{L^{\varphi}\left(\mathbb{R}^{n}\right)}^{-1}\right)}>\beta\right\},\left\|\mathbf{1}_{Q}\right\|_{L^{\varphi}\left(\mathbb{R}^{n}\right)}^{-1}\right) \\
\leq & C_{3}\left[1+\frac{\beta}{\|f\|_{\mathcal{L}_{A}^{\varphi, 1, s}\left(\mathbb{R}^{n}\right)}\left\|\mathbf{1}_{Q}\right\|_{L^{\varphi}\left(\mathbb{R}^{n}\right)}}\right]^{-q^{\prime}} .
\end{aligned}
$$

Remark 6.7. When $A$ is as in Remark 6.6(i), Theorem 6.7 coincides with [70, Theorem 2.5]. Moreover,

(i) if, for any $x \in \mathbb{R}^{n}$ and $t \in(0, \infty), \varphi(x, t):=t$ and $s=0$, then, by Theorem 3.1, we know that there exist two positive constants $C$ and $\widetilde{C}$ such that, for any $f \in$ $\operatorname{BMO}\left(\mathbb{R}^{n}\right)$, cube $Q \subset \mathbb{R}^{n}$ and $\beta \in(0, \infty)$,

$$
\left|\left\{x \in Q:\left|f(x)-f_{Q}\right|>\beta\right\}\right| \leq C e^{-\widetilde{C} \beta /\|f\|_{\text {BMO }\left(\mathbb{R}^{n}\right)}}|Q|,
$$

which is the well-known John-Nirenberg inequality obtained by John and Nirenberg [56];

(ii) if $\varphi$ is as in Remark 6.6(i) $)_{1}$, then Theorem 3.1 was obtained by Li [69];

(iii) if, for any $x \in \mathbb{R}^{n}$ and $t \in(0, \infty), \varphi(x, t):=w(x) t$ with $w$ as in Remark 6.6(i) $)_{2}$ and $s=0$, then Theorem 6.7 is the John-Nirenberg inequality for functions from the weighted $\mathrm{BMO}$ space $\mathrm{BMO}_{w}\left(\mathbb{R}^{n}\right)$, which was established by Muckenhoupt and Wheeden [86]. 
As a corollary of Theorem 6.7, we have the following conclusion, which shows that the space $\mathcal{L}_{A}^{\varphi, q, s}\left(\mathbb{R}^{n}\right)$ is independent of the choice of the index $q$ as long as $q \in\left[1,[q(\varphi)]^{\prime}\right)$; see [74, Lemma 3.6 and Theorem 3.8].

Theorem 6.8. Let $\varphi$ be as in Definition 6.2, $s \in \mathbb{Z}_{+}$and $q \in\left[1,[q(\varphi)]^{\prime}\right)$, where $q(\varphi)$ is as in (6.2). Then, for any $f \in L_{\text {loc }}^{1}\left(\mathbb{R}^{n}\right)$, the following statements are mutually equivalent:

(i)

$$
\|f\|_{\mathcal{L}_{A}^{\varphi, 1, s}\left(\mathbb{R}^{n}\right)}:=\sup _{Q \in \mathcal{Q}} \frac{1}{\left\|\mathbf{1}_{Q}\right\|_{L^{\varphi}\left(\mathbb{R}^{n}\right)}} \int_{Q}\left|f(x)-P_{Q}^{s} f(x)\right| d x<\infty ;
$$

(ii)

$$
\begin{aligned}
\|f\|_{\mathcal{L}_{A}^{\varphi, q, s}\left(\mathbb{R}^{n}\right)} & :=\sup _{Q \in \mathcal{Q}} \frac{1}{\left\|\mathbf{1}_{Q}\right\|_{L^{\varphi}\left(\mathbb{R}^{n}\right)}}\left\{\int_{Q}\left[\frac{\left|f(x)-P_{Q}^{s} f(x)\right|}{\varphi\left(x,\left\|\mathbf{1}_{Q}\right\|_{L^{\varphi}\left(\mathbb{R}^{n}\right)}^{-1}\right)}\right]^{q} \varphi\left(x,\left\|\mathbf{1}_{Q}\right\|_{L^{\varphi}\left(\mathbb{R}^{n}\right)}^{-1}\right) d x\right\}^{1 / q} \\
& <\infty ;
\end{aligned}
$$

(iii)

$$
\begin{aligned}
\|f\|_{\mathcal{L}_{A}^{\varphi, q, s}\left(\mathbb{R}^{n}\right)}^{(*)}:= & \sup _{Q \in \mathcal{Q}} \frac{1}{\left\|\mathbf{1}_{Q}\right\|_{L^{\varphi}\left(\mathbb{R}^{n}\right)}}\left\{\inf _{P \in \mathcal{P}_{s}\left(\mathbb{R}^{n}\right)} \int_{Q}\left[\frac{|f(x)-P(x)|}{\varphi\left(x,\left\|\mathbf{1}_{Q}\right\|_{L^{\varphi}\left(\mathbb{R}^{n}\right)}^{-1}\right)}\right]^{q}\right. \\
& \left.\times \varphi\left(x,\left\|\mathbf{1}_{Q}\right\|_{L^{\varphi}\left(\mathbb{R}^{n}\right)}^{-1}\right) d x\right\}^{1 / q}<\infty,
\end{aligned}
$$

where $Q$ is as in (6.26).

Let $r \in(1, \infty]$. A function $\varphi: \mathbb{R}^{n} \times[0, \infty) \rightarrow[0, \infty)$ is said to satisfy the anisotropic uniformly reverse Hölder condition, denoted by $\varphi \in \mathcal{R H}_{r}(A):=\mathcal{R H}_{r}\left(\mathbb{R}^{n} ; A\right)$, if, when $r \in(1, \infty)$,

$$
\sup _{t \in(0, \infty)} \sup _{B \in \mathfrak{B}}\left\{\frac{1}{|B|} \int_{B} \varphi(y, t) d y\right\}^{-1}\left\{\frac{1}{|B|} \int_{B}[\varphi(y, t)]^{r} d y\right\}^{1 / r}<\infty
$$

and, when $r=\infty$,

$$
\sup _{t \in(0, \infty)} \sup _{B \in \mathfrak{B}}\left\{\frac{1}{|B|} \int_{B} \varphi(y, t) d y\right\}^{-1}\{\underset{y \in B}{\operatorname{ess} \sup }[\varphi(y, t)]\}<\infty,
$$

where $\mathfrak{B}$ is as in (2.1). Moreover, $\bigcup_{r \in(1, \infty]} \mathcal{R H}_{r}(A)=\bigcup_{p \in[1, \infty)} \mathcal{A}_{p}(A)=\mathcal{A}_{\infty}(A)$ (see, for instance, [73,115]). Then, for any $\varphi \in \mathcal{A}_{\infty}(A)$, let

$$
r(\varphi):=\sup \left\{r \in(1, \infty]: \varphi \in \mathcal{R H}_{r}(A)\right\} .
$$

For any $r \in(0, \infty)$ and $s \in \mathbb{Z}_{+}$, let $L_{c, s}^{r}\left(\mathbb{R}^{n}\right)$ be the set of all $f \in L^{r}\left(\mathbb{R}^{n}\right)$, with compact support, satisfying that, for any multi-index $\alpha \in \mathbb{Z}_{+}^{n}$ with $|\alpha| \leq s, \int_{\mathbb{R}^{n}} f(x) x^{\alpha} d x=0$. The following dual space of $H_{A}^{\varphi}\left(\mathbb{R}^{n}\right)$ was given in [74, Theorem 3.17]. 
Theorem 6.9. Let $\varphi$ be as in Definition 6.2 and $s \in[m(\varphi), \infty) \cap \mathbb{Z}_{+}$, where $m(\varphi)$ is as in (6.3). Then the dual space of $H_{A}^{\varphi}\left(\mathbb{R}^{n}\right)$, denoted by $\left(H_{A}^{\varphi}\left(\mathbb{R}^{n}\right)\right)^{*}$, is $\mathcal{L}_{A}^{\varphi, 1, s}\left(\mathbb{R}^{n}\right)$ in the following sense:

(i) Let $g \in \mathcal{L}_{A}^{\varphi, 1, s}\left(\mathbb{R}^{n}\right)$. Then the linear functional

$$
L_{g}: f \longmapsto L_{g}(f):=\int_{\mathbb{R}^{n}} f(x) g(x) d x,
$$

initially defined for any $f \in L_{c, s}^{r}\left(\mathbb{R}^{n}\right)$, where $r \in\left(q(\varphi)[r(\varphi)]^{\prime}, \infty\right)$ with $q(\varphi)$ and $r(\varphi)$, respectively, as in (6.2) and (6.27), has a bounded extension to $H_{A}^{\varphi}\left(\mathbb{R}^{n}\right)$.

(ii) Conversely, every continuous linear functional on $H_{A}^{\varphi}\left(\mathbb{R}^{n}\right)$ arises as in (6.28) with a unique $g \in \mathcal{L}_{A}^{\varphi, 1, s}\left(\mathbb{R}^{n}\right)$.

Moreover, there exists a positive constant $C$ such that, for any $g \in \mathcal{L}_{A}^{\varphi, 1, s}\left(\mathbb{R}^{n}\right)$,

$$
\frac{1}{C}\|g\|_{\mathcal{L}_{A}^{\varphi, 1, s}\left(\mathbb{R}^{n}\right)} \leq\left\|L_{g}\right\|_{\left(H_{A}^{\varphi}\left(\mathbb{R}^{n}\right)\right)^{*}} \leq C\|g\|_{\mathcal{L}_{A}^{\varphi, 1, s}\left(\mathbb{R}^{n}\right)} .
$$

Remark 6.8. (i) When $A$ is as in Remark 6.6(i), by Theorem 6.9, we know that [70, Theorem 3.5] holds true. Moreover,

(i) $)_{1}$ when $\varphi$ is as in Remark 6.6(i) $)_{1}$, Theorem 6.9 was proved by Taibleson and Weiss [100];

(i) $)_{2}$ when $\varphi$ is as in Remark 6.6(i) $)_{2}$, Theorem 6.9 was obtained by GarcíaCuerva [43] for the case when $n=1$.

(iii) When $s=0$ (namely, when $\mathcal{L}_{A}^{\varphi, 1, s}\left(\mathbb{R}^{n}\right)=\mathrm{BMO}_{A}^{\varphi}\left(\mathbb{R}^{n}\right)$; see Remark 6.6(ii)), Theorem 6.9 goes back to [33, Lemma 2.4].

(iv) When $\varphi$ is as in Remark 6.6(iii), Theorem 6.9 coincides with [12, p. 51, Theorem 8.3].

Corollary 6.1. Let $\varphi$ be as in Definition 6.2. Then, for any $q \in\left[1,[q(\varphi)]^{\prime}\right)$ and $s \in[m(\varphi), \infty) \cap$ $\mathbb{Z}_{+}, \mathcal{L}_{A}^{\varphi, q, s}\left(\mathbb{R}^{n}\right)$ and $\mathcal{L}_{A}^{\varphi, 1, m(\varphi)}\left(\mathbb{R}^{n}\right)$ coincide with equivalent quasi-norms, where $q(\varphi)$ and $m(\varphi)$ are, respectively, as in (6.2) and (6.3).

Next, we present the characterizations of $H_{A}^{\varphi}\left(\mathbb{R}^{n}\right)$ in terms of wavelets. Recall that the following adapted anisotropic Musielak-Orlicz atoms and atomic Hardy spaces were introduced in [74, Definition 4.1].

Definition 6.10. Let $\varphi$ be as in Definition 6.2 and $q(\varphi)$ and $r(\varphi)$, respectively, as in (6.2) and (6.27).

(i) An anisotropic triplet $(\varphi, q, s)$ is said to be adapted admissible if $q \in\left(q(\varphi)[r(\varphi)]^{\prime}, \infty\right]$ and $s \in \mathbb{Z}_{+} \cap[m(\varphi), \infty)$ with $m(\varphi)$ as in (6.3). 
(ii) For any given adapted admissible triplet $(\varphi, q, s)$, a measurable function $a$ on $\mathbb{R}^{n}$ is called an adapted anisotropic Musielak-Orlicz $(\varphi, q, s)$-atom (shortly, a $(\varphi, q, s) \mathrm{ad}^{-}$ atom) if (ii) $)_{1}$ and (ii) $)_{3}$ of Definition 6.6 hold true and, instead of Definition 6.6(ii) $)_{2}, a$ satisfies

$$
\|a\|_{L^{q}\left(\mathbb{R}^{n}\right)} \leq \frac{|B|^{1 / q}}{\left\|\mathbf{1}_{B}\right\|_{L^{\varphi}\left(\mathbb{R}^{n}\right)}}
$$

(iii) For any given adapted admissible triplet $(\varphi, q, s)$, the adapted anisotropic Musielak-Orlicz atomic Hardy space $H_{\mathrm{ad}, A}^{\varphi, q, s}\left(\mathbb{R}^{n}\right)$ is defined to be the set of all $f \in \mathcal{S}^{\prime}\left(\mathbb{R}^{n}\right)$ satisfying that there exist $\left\{\lambda_{i}\right\}_{i \in \mathbb{N}} \subset \mathbb{C}$ and a sequence of $(\varphi, q, s)_{\mathrm{ad}^{-}}$ atoms, $\left\{a_{i}\right\}_{i \in \mathbb{N}}$, supported, respectively, in $\left\{B^{(i)}\right\}_{i \in \mathbb{N}} \subset \mathfrak{B}$ such that

$$
\begin{aligned}
& f=\sum_{i \in \mathbb{N}} \lambda_{i} a_{i} \text { in } \mathcal{S}^{\prime}\left(\mathbb{R}^{n}\right), \\
& \sum_{i \in \mathbb{N}} \varphi\left(B^{(i)},\left|\lambda_{i}\right|\left\|\mathbf{1}_{B^{(i)}}\right\|_{L^{\varphi}\left(\mathbb{R}^{n}\right)}^{-1}\right)<\infty .
\end{aligned}
$$

Moreover, for any $f \in H_{\mathrm{ad}, A}^{\varphi, q, s}\left(\mathbb{R}^{n}\right)$, let

$$
\|f\|_{H_{\mathrm{ad}, A}^{\varphi, q, s}\left(\mathbb{R}^{n}\right)}:=\inf \left\{\widetilde{\Lambda}\left(\left\{\lambda_{i} a_{i}\right\}_{i \in \mathbb{N}}\right)\right\}
$$

with the infimum being taken over all the decompositions of $f$ as above, where

$$
\widetilde{\Lambda}\left(\left\{\lambda_{i} a_{i}\right\}_{i \in \mathbb{N}}\right):=\inf \left\{\lambda \in(0, \infty): \sum_{i \in \mathbb{N}} \varphi\left(B^{(i)}, \frac{\left|\lambda_{i}\right|}{\lambda\left\|\mathbf{1}_{B^{(i)}}\right\|_{L^{\varphi}\left(\mathbb{R}^{n}\right)}}\right) \leq 1\right\} .
$$

In [74, Theorem 4.2], the authors established a new atomic characterization of $H_{A}^{\varphi}\left(\mathbb{R}^{n}\right)$ as follows, which is different from the one obtained in [68, Theorem 40] and also plays a key role in the proof of Theorem 6.11 below.

Theorem 6.10. Let $(\varphi, q, s)$ be adapted admissible. Then

$$
H_{A}^{\varphi}\left(\mathbb{R}^{n}\right)=H_{\mathrm{ad}, A}^{\varphi, q, s}\left(\mathbb{R}^{n}\right)
$$

with equivalent quasi-norms.

Remark 6.9. Recall that $\mathrm{Li}$ et al. [68, Theorem 40] also established an atomic characterization of $H_{A}^{\varphi}\left(\mathbb{R}^{n}\right)$ (see also [60] for the case $A=d \mathrm{I}_{n \times n}$ ). The size conditions of the atoms used in [68] are as in Definition 6.6(ii) $)_{2}$, but the size conditions of atoms used in Theorem 6.10 are as in Definition 6.10(ii). This is the difference between these two atomic characterizations. 
For any $L \in \mathbb{N}$, let

$$
\Gamma(L):=\left\{(\ell, j, k): \ell \in\{1, \cdots, L\}, j \in \mathbb{Z} \text { and } k \in \mathbb{Z}^{n}\right\}
$$

and, for any $f \in L^{2}\left(\mathbb{R}^{n}\right), j \in \mathbb{Z}, k \in \mathbb{Z}^{n}$ and $x \in \mathbb{R}^{n}$,

$$
f_{j, k}(x):=b^{j / 2} f\left(A^{j} x-k\right) .
$$

The following Definitions 6.11 and 6.12 are just, respectively, [12, p. 94, Definitions 4.1 and 4.2 and p. 84, Definition 1.3].

Definition 6.11. (i) Let $\mathcal{H}$ be a Hilbert space. A subset $X \subset \mathcal{H}$ is called a frame if there exist two positive constants $C$ and $\widetilde{C}$ such that, for any $f \in \mathcal{H}$,

$$
C\|f\|_{\mathcal{H}} \leq\left[\sum_{g \in X}|\langle f, g\rangle|^{2}\right]^{1 / 2} \leq \widetilde{C}\|f\|_{\mathcal{H}}
$$

Moreover, a frame is said to be tight if (6.31) holds true with $C=\widetilde{C}$.

(ii) Let $L \in \mathbb{N}$. A tight frame multiwavelet is a finite sequence of $L^{2}\left(\mathbb{R}^{n}\right)$ functions, $\left\{\psi^{(\ell)}\right\}_{\ell=1}^{L}$, satisfying $\left\{\psi_{j, k}^{(\ell)}:(\ell, j, k) \in \Gamma(L)\right\}$ forms a tight frame with the constant $C=1$ for $L^{2}\left(\mathbb{R}^{n}\right)$, where $\Gamma(L)$ and $C$ are, respectively, as in (6.29) and (6.31) and, for any $(\ell, j, k) \in \Gamma(L), \psi_{j, k}^{(\ell)}$ is as in (6.30) with $f$ replaced by $\psi^{(\ell)}$.

Definition 6.12. Let $r \in \mathbb{Z}_{+}$. An $r$ - regular function $f$ is a $C^{r}\left(\mathbb{R}^{n}\right)$ function satisfying, for any $i \in \mathbb{N}$ and multi-index $\alpha \in \mathbb{Z}_{+}^{n}$ with $|\alpha| \leq r$, there exists a positive constant $C_{(i, \alpha)}$, depending on $i$ and $\alpha$, such that, for any $x \in \mathbb{R}^{n}$,

$$
\left|\partial^{\alpha} f(x)\right| \leq C_{(i, \alpha)}(1+|x|)^{-i}
$$

Furthermore, for any $L \in \mathbb{N}$, a tight frame multiwavelet $\left\{\psi^{(\ell)}\right\}_{\ell=1}^{L}$ is said to be $r$ - regular if, for any $\ell \in\{1, \cdots, L\}, \psi^{(\ell)}$ is an $r$-regular function.

Moreover, we can define an unconditional basis of $H_{A}^{\varphi}\left(\mathbb{R}^{n}\right)$ in terms of $r$-regular tight frame multiwavelets as follows.

Definition 6.13. Let $\varphi$ be as in Definition 6.2, $L \in \mathbb{N}$ and $r \in \mathbb{Z}_{+}$. Assume that $\left\{\psi^{(\ell)}\right\}_{\ell=1}^{L}$ is an $r$-regular tight frame multiwavelet. The sequence $\left\{\psi_{j, k}^{(\ell)}:(\ell, j, k) \in \Gamma(L)\right\}$ is called an unconditional basis of $H_{A}^{\varphi}\left(\mathbb{R}^{n}\right)$ if, for any $f \in H_{A}^{\varphi}\left(\mathbb{R}^{n}\right)$,

$$
f=\sum_{(\ell, j, k) \in \Gamma(L)}\left\langle f, \psi_{j, k}^{(\ell)}\right\rangle \psi_{j, k}^{(\ell)} \quad \text { in } H_{A}^{\varphi}\left(\mathbb{R}^{n}\right),
$$

where the convergence is unconditional, namely, for an arbitrary permutation of $\Gamma(L)$, denoted by $\widetilde{\Gamma}(L)$, the equality (6.32), with $\Gamma(L)$ replaced by $\widetilde{\Gamma}(L)$, also holds true. 
Remark 6.10. Let $\varphi, r, L$ and $\left\{\psi^{(\ell)}\right\}_{\ell=1}^{L}$ be as in Definition 6.13. Then, by [12, p. 94, Theorem 4.2] and [74, Proposition 4.14], we know that, for any $(\ell, j, k) \in \Gamma(L), \psi_{j, k}^{(\ell)} \in$ $\mathcal{L}_{A}^{\varphi, 1, s}\left(\mathbb{R}^{n}\right)$. Thus, for any $f \in H_{A}^{\varphi}\left(\mathbb{R}^{n}\right) \subset\left(\mathcal{L}_{A}^{\varphi, 1, s}\left(\mathbb{R}^{n}\right)\right)^{*}$ and $(\ell, j, k) \in \Gamma(L),\left\langle f, \psi_{j, k}^{(\ell)}\right\rangle$ makes sense.

The following conclusion was obtained in [74, Theorem 25].

Theorem 6.11. Let $\varphi$ be as in Definition 6.2 and $r \in \mathbb{N}$ satisfy

$$
\frac{q(\varphi)}{i(\varphi)}-1<\frac{\left(\ln \lambda_{-}\right)^{2}}{\ln b \ln \lambda_{+}} r
$$

where $q(\varphi)$ and $i(\varphi)$ are, respectively, as in (6.2) and (6.1). Assume that $\left\{\psi^{(\ell)}\right\}_{\ell=1}^{L}$ with $L \in \mathbb{N}$ is an r-regular tight frame multiwavelet.

(i) If, for any $\ell \in\{1, \cdots, L\}$, the function $\psi^{(\ell)}$ has the vanishing moments up to the order $m(\varphi)$ which is as in (6.3), then, for any $f \in H_{A}^{\varphi}\left(\mathbb{R}^{n}\right)$, (6.32) with the convergence being unconditional holds true, and

$$
\begin{aligned}
\|f\|_{H_{A}^{\varphi}\left(\mathbb{R}^{n}\right)} & \sim\left\|\left[\sum_{(\ell, j, k) \in \Gamma(L)}\left|\left\langle f, \psi_{j, k}^{(\ell)}\right\rangle\right|^{2}\left|\psi_{j, k}^{(\ell)}\right|^{2}\right]^{1 / 2}\right\|_{L^{\varphi}\left(\mathbb{R}^{n}\right)} \\
& \sim\left\|\left[\sum_{(\ell, j, k) \in \Gamma(L)}\left|\left\langle f, \psi_{j, k}^{(\ell)}\right\rangle\right|^{2}\left|\left(\mathbf{1}_{E_{\ell}}\right)_{j, k}\right|^{2}\right]^{1 / 2}\right\|_{L^{\varphi}\left(\mathbb{R}^{n}\right)}
\end{aligned}
$$

with the positive equivalence constants independent of $f$, where, for any $\ell \in\{1, \cdots, L\}$, $E_{\ell}$ is a bounded measurable subset of $\mathbb{R}^{n}$ with $\left|E_{\ell}\right|>0$ and, for any $j \in \mathbb{Z}$ and $k \in \mathbb{Z}^{n}$, $\left(\mathbf{1}_{E_{\ell}}\right)_{j, k}$ is as in (6.30).

(ii) If, with the assumption as in (i), $\left\{\psi^{(\ell)}\right\}_{\ell=1}^{L}$ is an orthonormal multiwavelet, then, for any $\left\{c_{j, k}^{(\ell)}\right\}_{(\ell, j, k) \in \Gamma(L)} \subset \mathbb{R}$ satisfying

$$
\left[\sum_{(\ell, j, k) \in \Gamma(L)}\left|c_{j, k}^{(\ell)}\right|^{2}\left|\psi_{j, k}^{(\ell)}\right|^{2}\right]^{1 / 2} \in L^{\varphi}\left(\mathbb{R}^{n}\right),
$$

there exists a unique $f \in H_{A}^{\varphi}\left(\mathbb{R}^{n}\right)$ such that, for any $(\ell, j, k) \in \Gamma(L), c_{j, k}^{(\ell)}=\left\langle f, \psi_{j, k}^{(\ell)}\right\rangle$ and (6.33) holds true.

Remark 6.11. (i) When $A:=d \mathrm{I}_{n \times n}$ for some $d \in \mathbb{R}$ with $|d| \in(1, \infty)$, the space $H_{A}^{\varphi}\left(\mathbb{R}^{n}\right)$ becomes the classical Musielak-Orlicz Hardy space $H^{\varphi}\left(\mathbb{R}^{n}\right)$ (see [60]). 
In this case, by Theorem 6.11, we obtain a kind of wavelet characterizations of $H^{\varphi}\left(\mathbb{R}^{n}\right)$. Recall that, very recently, Fu and Yang [42] also established another sort of wavelet characterizations of $H^{\varphi}\left(\mathbb{R}^{n}\right)$. We should point out that the wavelet system used in [42] to characterize the space $H^{\varphi}\left(\mathbb{R}^{n}\right)$ is required to have compact supports; however, this requirement is not needed for tight frame multiwavelet used in Theorem 6.11 to characterize the space $H_{A}^{\varphi}\left(\mathbb{R}^{n}\right)$.

(ii) Let $p \in(0,1]$. If, for any $x \in \mathbb{R}^{n}$ and $t \in(0, \infty), \varphi(x, t):=t^{p}$, then the space $H_{A}^{\varphi}\left(\mathbb{R}^{n}\right)$ becomes the anisotropic Hardy space $H_{A}^{p}\left(\mathbb{R}^{n}\right)$ of Bownik (see [12]) and $q(\varphi) / i(\varphi)=1 / p$. In this case, Theorem 6.11 goes back to [12, p. 109, Theorem 6.7].

(iii) We point out that the boundedness of maximal $\theta$-operators, defined by the way similar to (4.29), from $H_{A}^{\varphi}\left(\mathbb{R}^{n}\right)$ to $L^{\varphi}\left(\mathbb{R}^{n}\right)$ is still unknown. In addition, a remark similar to Remark 4.5(iii) should be also made for the space $H_{A}^{\varphi}\left(\mathbb{R}^{n}\right)$.

(iv) Let $\phi: \mathbb{R}^{n} \times[0, \infty) \rightarrow[0, \infty)$ be a generalized quasi- $\Phi$-function (see [48, Definition 2.1]). Recall that Ho [48] introduced a kind of Hardy-Musielak-Orlicz spaces $H^{\phi}\left(\mathbb{R}^{n}\right)$, which includes the classical variable Hardy space (see $\left.[23,87]\right)$ as a special case. Observe that, formally, the anisotropic Musielak-Orlicz Hardy space $H_{A}^{\varphi}\left(\mathbb{R}^{n}\right)$ includes the anisotropic variable Hardy space $H_{A}^{p(\cdot)}\left(\mathbb{R}^{n}\right)$ as a special case, but actually they cannot cover each other due to different assumptions, respectively, on $\varphi$ and $p(\cdot)$ (see Remark 6.1(ii)). An interesting and challenging question is to find some reasonable assumptions on $\varphi$ such that the real-variable theory on $H_{A}^{\varphi}\left(\mathbb{R}^{n}\right)$ completely covers the corresponding one of the space $H_{A}^{p(\cdot)}\left(\mathbb{R}^{n}\right)$ as a special case.

\section{Acknowledgements}

The authors would like to thank the referee for her/his carefully reading and useful comments which improve the presentation of this article. Jun Liu was supported by the Scientific Research Foundation of China University of Mining and Technology (Grant No. 102519054). Dorothee D. Haroske was partially supported by the German Research Foundation (DFG) (Grant No. Ha 2794/8-1). Dachun Yang was supported by the National Natural Science Foundation of China (Grant Nos. 11761131002, 11971058 and 11671185).

\section{References}

[1] W. Abu-Shammala and A. Torchinsky, The Hardy-Lorentz spaces $H^{p, q}\left(\mathbb{R}^{n}\right)$, Studia Math., 182 (2007), 283-294.

[2] V. Almeida, J. J. Betancor and L. Rodríguez-Mesa, Anisotropic Hardy-Lorentz spaces with variable exponents, Canad. J. Math., 69 (2017), 1219-1273.

[3] J. Álvarez and M. Milman, $H^{p}$ continuity properties of Calderón-Zygmund-type operators, J. Math. Anal. Appl., 118 (1986), 63-79. 
[4] B. Barrios and J. J. Betancor, Characterizations of anisotropic Besov spaces, Math. Nachr., 284 (2011), 1796-1819.

[5] B. Barrios and J. J. Betancor, Anisotropic weak Hardy spaces and wavelets, J. Funct. Spaces Appl., 2012, Art. ID 809121, 17 pages.

[6] J. Bergh and J. Löfström, Interpolation Spaces, An Introduction, Grundlehren der Mathematischen Wissenschaften, 223, Springer-Verlag, Berlin-New York, 1976.

[7] M. Z. Berkolaǐko and I. Ya. Novikov, Unconditional bases in spaces of functions of anisotropic smoothness, (Russian) Trudy Mat. Inst. Steklov., 204 (1993), Issled. po Teor. Differ. Funktsiŭ Mnogikh Peremen. i ee Prilozh. 16, 35-51; translation in Proc. Steklov Inst. Math., 3(204) (1994), 27-41.

[8] M. Z. Berkolaǐko and I. Ya. Novikov, Bases of wavelets, and linear operators in anisotropic Lizorkin-Triebel spaces, (Russian) in: in honor of the ninetieth birthday of Academician S. M. Nikol'skiǔ (Russian), Trudy Mat. Inst. Steklov., 210 (1995), Teor. Funktsiǐi Differ. Uravn., 5-30.

[9] O. V. Besov, V. P. Il'in and S. M. Nikol'skiĭ, Integral Representations of Functions, and Embedding Theorems, Vol. I. Translated from the Russian, Scripta Series in Mathematics, Edited by Mitchell H. Taibleson, V. H. Winston \& Sons, Washington, D.C.; Halsted Press (John Wiley \& Sons), New York-Toronto, Ont.-London, 1978, viii+345 pages.

[10] O. V. Besov, L. D. Kudryavtsev, P. I. Lizorkin and S. M. Nikol'skiĭ, Investigations in the theory of spaces of differentiable functions of several variables, (Russian) Translated in Proc. Steklov Inst. Math., 1990, no. 1, 73-139, Probability theory, function theory, mechanics (Russian), Trudy Mat. Inst. Steklov., 182 (1988), 68-127.

[11] J. J. Betancor and W. Damián, Anisotropic local Hardy spaces, J. Fourier Anal. Appl., 16 (2010), 658-675.

[12] M. Bownik, Anisotropic Hardy Spaces and Wavelets, Mem. Amer. Math. Soc., 164(781) (2003), vi+122pages.

[13] M. Bownik, Atomic and molecular decompositions of anisotropic Besov spaces, Math. Z., 250 (2005), 539-571.

[14] M. Bownik, Anisotropic Triebel-Lizorkin spaces with doubling measures, J. Geom. Anal., 17 (2007), 387-424.

[15] M. Bownik and K.-P. Ho, Atomic and molecular decompositions of anisotropic TriebelLizorkin spaces, Trans. Amer. Math. Soc., 358 (2006), 1469-1510.

[16] M. Bownik, B. Li, D. Yang and Y. Zhou, Weighted anisotropic Hardy spaces and their applications in boundedness of sublinear operators, Indiana Univ. Math. J., 57 (2008), 3065-3100.

[17] M. Bownik, B. Li, D. Yang and Y. Zhou, Weighted anisotropic product Hardy spaces and boundedness of sublinear operators, Math. Nachr., 283 (2010), 392-442.

[18] M. Bownik and L.-A. D. Wang, A PDE characterization of anisotropic Hardy spaces, arXiv: 2011.10651.

[19] A.-P. Calderón and A. Torchinsky, Parabolic maximal functions associated with a distribution, Adv. Math., 16 (1975), 1-64.

[20] S. Campanato, Proprietà di una famiglia di spazi funzionali, Ann. Scuola Norm. Sup. Pisa (3), 18 (1964), 137-160.

[21] M. Christ, A $T(b)$ theorem with remarks on analytic capacity and the Cauchy integral, Colloq. Math., 60/61 (1990), 601-628.

[22] D. V. Cruz-Uribe and A. Fiorenza, Variable Lebesgue Spaces. Foundations and Harmonic Analysis, Applied and Numerical Harmonic Analysis, Birkhäuser/Springer, Heidelberg, 2013. 
[23] D. Cruz-Uribe and L.-A. D. Wang, Variable Hardy spaces, Indiana Univ. Math. J., 63 (2014), 447-493.

[24] S. Dachkovski, Anisotropic function spaces and related semi-linear hypoelliptic equations, Math. Nachr., 248/249 (2003), 40-61.

[25] S. Dekel, Y. Han and P. Petrushev, Anisotropic meshless frames on $\mathbb{R}^{n}$, J. Fourier Anal. Appl., 15 (2009), 634-662.

[26] L. Diening, P. Harjulehto, P. Hästö and M. Růžička, Lebesgue and Sobolev Spaces with Variable Exponents, Lecture Notes in Math., 2017, Springer, Heidelberg, 2011.

[27] Y. Ding and S. Lan, Anisotropic weak Hardy spaces and interpolation theorems, Sci. China Ser. A, 51 (2008), 1690-1704.

[28] P. Dintelmann, On Fourier multipliers between Anisotropic Weighted Function Spaces, PhD thesis, TH Darmstadt, 1995.

[29] P. Dintelmann, Fourier multipliers between weighted anisotropic function spaces. I. Besov spaces, Z. Anal. Anwend., 15 (1996), 579-601.

[30] P. Dintelmann, Fourier multipliers between weighted anisotropic function spaces. II. BesovTriebel spaces, Z. Anal. Anwend., 15 (1996), 799-818.

[31] L. Ephremidze, V. Kokilashvili and S. Samko, Fractional, maximal and singular operators in variable exponent Lorentz spaces, Fract. Calc. Appl. Anal., 11 (2008), 407-420.

[32] X. Fan, J. He, B. Li and D. Yang, Real-variable characterizations of anisotropic product Musielak-Orlicz Hardy spaces, Sci. China Math., 60 (2017), 2093-2154.

[33] X. Fan and B. Li, Anisotropic tent spaces of Musielak-Orlicz type and their applications, Adv. Math. (China), 45 (2016), 233-251.

[34] W. Farkas, Atomic and subatomic decompositions in anisotropic function spaces, Math. Nachr., 209 (2000), 83-113.

[35] W. Farkas, Eigenvalue distribution of some fractal semi-elliptic differential operator, Math. Z., 236 (2001), 291-320.

[36] W. Farkas, J. Johnsen and W. Sickel, Traces of anisotropic Besov-Lizorkin-Triebel spaces-a complete treatment of the borderline cases, Math. Bohem., 125 (2000), 1-37.

[37] C. Fefferman, N. M. Rivière and Y. Sagher, Interpolation between $H^{p}$ spaces: the real method, Trans. Amer. Math. Soc., 191 (1974), 75-81.

[38] C. Fefferman and E. M. Stein, $H^{p}$ spaces of several variables, Acta Math., 129 (1972), 137-193.

[39] R. Fefferman and F. Soria, The spaces weak $H^{1}$, Studia Math., 85 (1987), 1-16.

[40] M. Frazier and B. Jawerth, A discrete transform and decomposition of distribution spaces, J. Funct. Anal., 93 (1990), 34-170.

[41] M. Frazier, B. Jawerth and G. Weiss, Littlewood-Paley theory and the study of function spaces, CBMS Regional Conference Series in Mathematics, 79, Published for the Conference Board of the Mathematical Sciences, Washington, DC; by the American Mathematical Society, Providence, RI, 1991, viii+132pages.

[42] X. Fu and D. Yang, Wavelet characterizations of Musielak-Orlicz Hardy spaces, Banach J. Math. Anal., 12 (2018), 1017-1046.

[43] J. García-Cuerva, Weighted $H^{p}$ spaces, Dissertationes Math. (Rozprawy Mat.), 162 (1979), $1-63$.

[44] G. Garrigós, R. Hochmuth and A. Tabacco, Wavelet characterizations for anisotropic Besov spaces with $0<p<1$, Proc. Edinb. Math. Soc(2)., 47 (2004), 573-595.

[45] G. Garrigós and A. Tabacco, Wavelet decompositions of anisotropic Besov spaces, Math. Nachr., 239/240 (2002), 80-102.

[46] L. Grafakos, Classical Fourier Analysis, Third edition, Graduate Texts in Mathematics, 249, 
Springer, New York, 2014.

[47] D. D. Haroske and E. Tamási, Wavelet frames for distributions in anisotropic Besov spaces, Georgian Math. J., 12 (2005), 637-658.

[48] K.-P. Ho, Intrinsic atomic and molecular decompositions of Hardy-Musielak-Orlicz spaces, Banach J. Math. Anal., 10 (2016), 566-592.

[49] R. Hochmuth, Wavelet characterizations for anisotropic Besov spaces, Appl. Comput. Harmon. Anal., 12 (2002), 179-208.

[50] S. Hou, D. Yang and S. Yang, Lusin area function and molecular characterizations of Musielak-Orlicz Hardy spaces and their applications, Commun. Contemp. Math., 15 (2013), 1350029.

[51] L. Huang, J. Liu, D. Yang and W. Yuan, Real-variable characterizations of new anisotropic mixed-norm Hardy spaces, Commun. Pure Appl. Anal., 19 (2020), 3033-3082.

[52] L. Huang and D. Yang, On function spaces with mixed norms-a survey, J. Math. Study, (2021), doi: 10.4208/jms.v54n3.21.03.

[53] T. Jakab and M. Mitrea, Parabolic initial boundary value problems in nonsmooth cylinders with data in anisotropic Besov spaces, Math. Res. Lett., 13 (2006), 825-831.

[54] S. Janson, Generalizations of Lipschitz spaces and an application to Hardy spaces and bounded mean oscillation, Duke Math. J., 47 (1980), 959-982.

[55] Y. Jiang, Approximation of anisotropic classes by wavelets, J. Math. Anal. Appl., 318 (2006), $112-119$.

[56] F. John and L. Nirenberg, On functions of bounded mean oscillation, Commun. Pure Appl. Math., 14 (1961), 415-426.

[57] J. Johnsen, Pointwise multiplication of Besov and Triebel-Lizorkin spaces, Math. Nachr., 175 (1995), 85-133.

[58] J. Johnsen, S. Munch Hansen and W. Sickel, Anisotropic Lizorkin-Triebel spaces with mixed norms-traces on smooth boundaries, Math. Nachr., 288 (2015), 1327-1359.

[59] H. Kempka and J. Vybíral, Lorentz spaces with variable exponents, Math. Nachr., 287 (2014), 938-954.

[60] L. D. Ky, New Hardy spaces of Musielak-Orlicz type and boundedness of sublinear operators, Integral Equations Operator Theory, 78 (2014), 115-150.

[61] G. Kyriazis, Multilevel characterizations of anisotropic function spaces, SIAM J. Math. Anal., 36 (2004), 441-462.

[62] G. Kyriazis and P. Petrushev, On the construction of frames for Triebel-Lizorkin and Besov spaces, Proc. Amer. Math. Soc., 134 (2006), 1759-1770.

[63] B. Li, M. Bownik and D. Yang, Littlewood-Paley characterization and duality of weighted anisotropic product Hardy spaces, J. Funct. Anal., 266 (2014), 2611-2661.

[64] B. Li, M. Bownik, D. Yang, and W. Yuan, Duality of weighted anisotropic Besov and TriebelLizorkin spaces, Positivity, 16 (2012), 213-244.

[65] B. Li, M. Bownik, D. Yang, and W. Yuan, A mean characterization of weighted anisotropic Besov and Triebel-Lizorkin spaces, Z. Anal. Anwend., 33 (2014), 125-147.

[66] B. Li, X. Fan, Z. Fu and D. Yang, Molecular characterization of anisotropic Musielak-Orlicz Hardy spaces and their applications, Acta Math. Sin., 32 (2016), 1391-1414.

[67] B. Li, X. Fan and D. Yang, Littlewood-Paley characterizations of anisotropic Hardy spaces of Musielak-Orlicz type, Taiwanese J. Math., 19 (2015), 279-314.

[68] B. Li, D. Yang and W. Yuan, Anisotropic Hardy spaces of Musielak-Orlicz type with applications to boundedness of sublinear operators, The Scientific World Journal, Vol. 2014, Article ID 306214, 19 pages, 2014, DOI: 10.1155/2014/306214. 
[69] W. Li, John-Nirenberg type inequalities for the Morrey-Campanato spaces, J. Inequal. Appl., 2008, Art. ID 239414, 5 pages.

[70] Y. Liang and D. Yang, Musielak-Orlicz Campanato spaces and applications, J. Math. Anal. Appl., 406 (2013), 307-322.

[71] M. Liao, J. Li, Bo Li and B. Li, A new molecular characterization of diagonal anisotropic Musielak-Orlicz Hardy spaces, Bull. Sci. Math., to appear.

[72] H. Liu, The weak $H^{p}$ spaces on homogeneous groups, Harmonic Analysis (Tianjin, 1988), 113-118, Lecture Notes in Math. 1494, Springer, Berlin, 1991.

[73] J. Liu, D. D. Haroske and D. Yang, New molecular characterizations of anisotropic MusielakOrlicz Hardy spaces and their applications, J. Math. Anal. Appl., 475 (2019), 1341-1366.

[74] J. Liu, D. D. Haroske, D. Yang and W. Yuan, Dual spaces and their applications in wavelet characterizations of anisotropic Musielak-Orlicz Hardy spaces, Appl. Comput. Math., 19 (2020), 106-131.

[75] J. Liu, F. Weisz, D. Yang and W. Yuan, Variable anisotropic Hardy spaces and their applications, Taiwanese J. Math., 22 (2018), 1173-1216.

[76] J. Liu, F. Weisz, D. Yang and W. Yuan, Littlewood-Paley and finite atomic characterizations of anisotropic variable Hardy-Lorentz spaces and their applications, J. Fourier Anal. Appl., 25 (2019), 874-922.

[77] J. Liu, D. Yang and W. Yuan, Anisotropic Hardy-Lorentz spaces and their applications, Sci. China Math., 59 (2016), 1669-1720.

[78] J. Liu, D. Yang and W. Yuan, Anisotropic variable Hardy-Lorentz spaces and their real interpolation, J. Math. Anal. Appl., 456 (2017), 356-393.

[79] J. Liu, D. Yang and W. Yuan, Littlewood-Paley characterizations of anisotropic HardyLorentz spaces, Acta Math. Sci. Ser. B Engl. Ed., 38 (2018), 1-33.

[80] J. Liu, D. Yang and W. Yuan, Littlewood-Paley characterizations of weighted anisotropic Triebel-Lizorkin spaces via averages on balls I, Z. Anal. Anwend., 38 (2019), 397-418.

[81] J. Liu, D. Yang and W. Yuan, Littlewood-Paley characterizations of weighted anisotropic Triebel-Lizorkin spaces via averages on balls II, Z. Anal. Anwend., 39 (2020), 1-26.

[82] X. Liu, X. Qiu and B. Li, Molecular characterization of anisotropic variable Hardy-Lorentz spaces, Tohoku Math. J(2)., 72 (2020), 211-233.

[83] T. D. Long and H. Triebel, Equivalent norms and Schauder bases in anisotropic Besov spaces, Proc. Roy. Soc. Edinburgh Sect. A, 84 (1979), 177-183.

[84] C. Merucci, Applications of interpolation with a function parameter to Lorentz, Sobolev and Besov spaces, Interpolation Spaces and Allied Topics in Analysis (Lund, 1983), 183-201, Lecture Notes in Math., 1070, Springer, Berlin, 1984.

[85] S. D. Moura, I. Piotrowska and M. Piotrowski, Non-smooth atomic decompositions of anisotropic function spaces and some applications, Studia Math., 180 (2007), 169-190.

[86] B. Muckenhoupt and R. L. Wheeden, Weighted bounded mean oscillation and the Hilbert transform, Studia Math., 54 (1976), 221-237.

[87] E. Nakai and Y. Sawano, Hardy spaces with variable exponents and generalized Campanato spaces, J. Funct. Anal., 262 (2012), 3665-3748.

[88] S. M. Nikol'ski1̌, Approximation of Functions of Several Variables and Embedding Theorems, Nauka, Moscow, second, revised and supplemented edition, 1977, Russian; First edition 1969. Engl. Transl.: Grundlehren der Mathematischen Wissenschaften, vol. 205, Springer, New York-Heidelberg, 1975.

[89] N. M. Rivière and Y. Sagher, Interpolation between $L^{\infty}$ and $H^{1}$, the real method, J. Funct. Anal., 14 (1973), 401-409. 
[90] S. Sato, Characterization of parabolic Hardy spaces by Littlewood-Paley functions, Results Math., 73 (2018), Art. 106, 20 pages.

[91] Y. Sawano, Atomic decompositions of Hardy spaces with variable exponents and its application to bounded linear operator, Integral Equations Operator Theory, 77 (2013), 123-148.

[92] H.-J. Schmeisser, Anisotropic spaces. II. Equivalent norms for abstract spaces, function spaces with weights of Sobolev-Besov type, Math. Nachr., 79 (1977), 55-73.

[93] H.-J. Schmeisser and H. Triebel, Anisotropic spaces. I. Interpolation of abstract spaces and function spaces, Math. Nachr., 73 (1976), 107-123.

[94] H.-J. Schmeisser and H. Triebel, Topics in Fourier Analysis and Function Spaces, A WileyInterscience Publication, John Wiley \& Sons, Ltd., Chichester, 1987, 300 pages.

[95] A. Seeger, A note on Triebel-Lizorkin spaces, Approximation and function spaces (Warsaw, 1986), 391-400, Banach Center Publ., 22, PWN, Warsaw, 1989.

[96] E. M. Stein, Harmonic Analysis: Real-Variable Methods, Orthogonality, and Oscillatory Integrals, Princeton Mathematical Series 43, Monographs in Harmonic Analysis III, Princeton University Press, Princeton, NJ, 1993.

[97] E. M. Stein, M. H. Taibleson and G. Weiss, Weak type estimates for maximal operators on certain $H^{p}$ classes, Proceedings of the Seminar on Harmonic Analysis (Pisa, 1980), Rend. Circ. Mat. Palermo, 1981, suppl. 1, 81-97.

[98] J.-O. Strömberg, Bounded mean oscillation with Orlicz norms and duality of Hardy spaces, Indiana Univ. Math. J., 28 (1979), 511-544.

[99] R. Sun, J. Li and B. Li, Molecular characterization of anisotropic weak Musielak-Orlicz Hardy spaces and their applications, Commun. Pure Appl. Anal., 18 (2019), 2377-2395.

[100] M. H. Taibleson and G. Weiss, The molecular characterization of certain Hardy spaces, in: Representation theorems for Hardy spaces, pp. 67-149, Astérisque, 77, Soc. Math. France, Paris, 1980.

[101] E. Tamási, Eigenvalue distribution of semi-elliptic operators in anisotropic Sobolev spaces, Z. Anal. Anwend., 28 (2009), 233-248.

[102] H. Triebel, Interpolation Theory, Function Spaces, Differential Operators, North-Holland Mathematical Library, 18, North-Holland Publishing Co., Amsterdam-New York, 1978, 528 pages.

[103] H. Triebel, Theorems of Littlewood-Paley type for BMO and for anisotropic Hardy spaces, in: Constructive Function Theory, 77, 525-532, Publ. House Bulgar. Acad. Sci., Sofia, 1980.

[104] H. Triebel, Anisotropic function spaces. I. Hardy's inequality, decompositions, Anal. Math., 10 (1984), 53-77.

[105] H. Triebel, Anisotropic function spaces. II. Traces, Anal. Math., 10 (1984), 79-96.

[106] H. Triebel, Weighted anisotropic Sobolev spaces on domains: extensions and traces, Math. Nachr., 119 (1984), 309-319.

[107] H. Triebel, Wavelet frames for distributions; local and pointwise regularity, Studia Math., 154 (2003), 59-88.

[108] H. Triebel, Wavelet bases in anisotropic function spaces, in: J. Rákosník, editor, Function Spaces, Differential Operators and Nonlinear Analysis. Conference held in Milovy, 2004, 370-387, Math. Inst. Acad. Sci. Czech Republic, Prague, 2005.

[109] H. Triebel, Theory of Function Spaces. III, Birkhäuser Verlag, Basel, 2006.

[110] L.-A. D. Wang, A multiplier theorem on anisotropic Hardy spaces, Canad. Math. Bull., 61 (2018), 390-404.

[111] F. Weisz, Convergence and Summability of Fourier Transforms and Hardy Spaces, Birkhäuser, Basel, 2017. 
[112] M. Yamazaki, A quasihomogeneous version of paradifferential operators. I. Boundedness on spaces of Besov type, J. Fac. Sci. Univ. Tokyo Sect. IA Math., 33 (1986), 131-174.

[113] M. Yamazaki, A quasihomogeneous version of paradifferential operators. II. A symbol calculus, J. Fac. Sci. Univ. Tokyo Sect. IA Math., 33 (1986), 311-345.

[114] X. Yan, D. Yang, Y. Yuan and C. Zhuo, Variable weak Hardy spaces and thier applications, J. Funct. Anal., 271 (2016), 2822-2887.

[115] D. Yang, Y. Liang and L. D. Ky, Real-Variable Theory of Musielak-Orlicz Hardy Spaces, Lecture Notes in Mathematics 2182, Springer-Verlag, Cham, 2017.

[116] Da. Yang, Do. Yang and G. Hu, The Hardy Space $H^{1}$ with Non-doubling Measures and Their Applications, Lecture Notes in Mathematics 2084, Springer-Verlag, Cham, 2013.

[117] D. Yang and S. Yang, New characterizations of weighted Morrey-Campanato spaces, Taiwanese J. Math., 15 (2011), 141-163.

[118] K. Zhao and L. Li, Molecular decomposition of weighted anisotropic Hardy spaces, Taiwanese J. Math., 17 (2013), 583-599.

[119] C. Zhuo, Y. Sawano and D. Yang, Hardy spaces with variable exponents on RD-spaces and applications, Dissertationes Math. (Rozprawy Mat.), 520 (2016), 1-74.

[120] C. Zhuo, D. Yang and W. Yuan, Interpolation between $H^{p(\cdot)}\left(\mathbb{R}^{n}\right)$ and $L^{\infty}\left(\mathbb{R}^{n}\right)$ : real method, J. Geom. Anal., 28 (2018), 2288-2311. 